\title{
Acene-linked Zethrenes and Bisphenalenyls: a DFT Search for Organic Tetraradicals
}

Vladimir I. Minkin, Andrey G. Starikov*, Alyona A. Starikova

Institute of Physical and Organic Chemistry at Southern Federal University, 344090 Rostov-on-Don, Russian Federation

\section{Corresponding Author}

*Email: agstarikov@sfedu.ru

\section{Table of Contents}

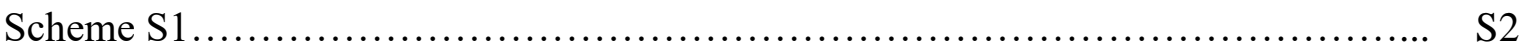

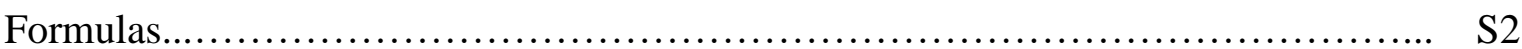

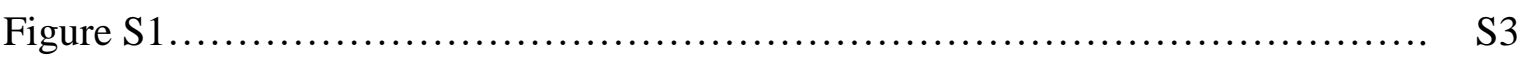

A sample of input for calculating the BS state............................... S3

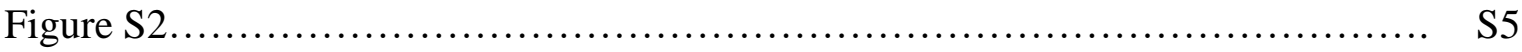

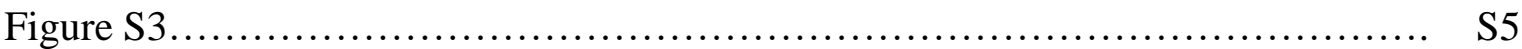

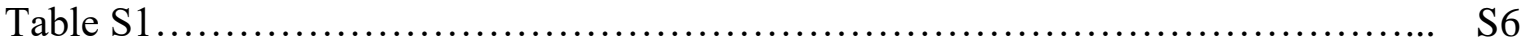

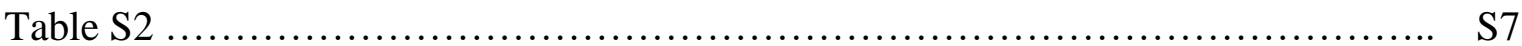

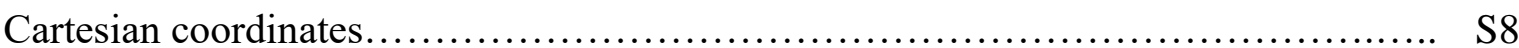



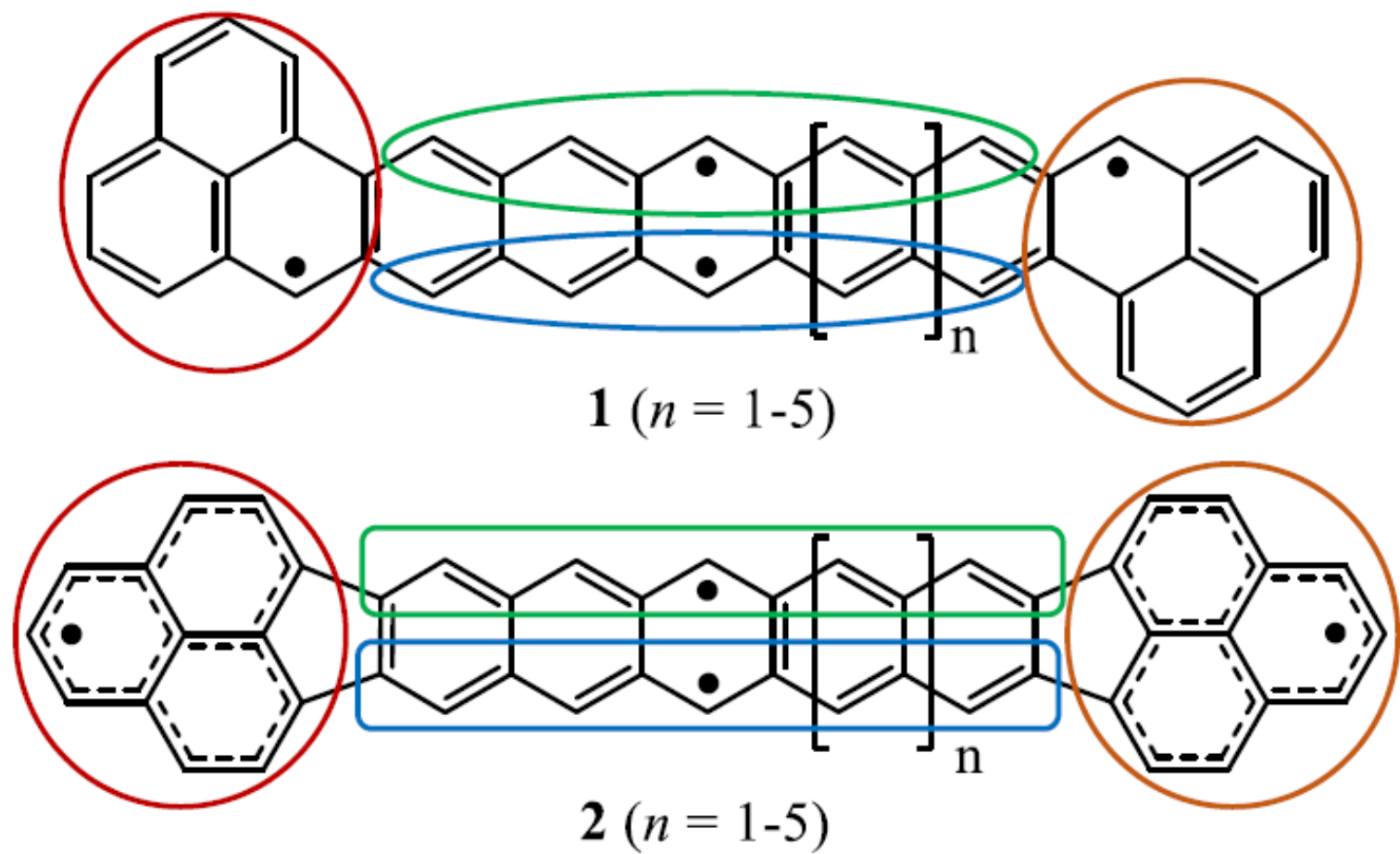

Scheme S1. Schematic representation of partitioning into the fragments (fragment 1 - red, fragment 2 - green, fragment 3 - blue, fragment 4 - orange) of compounds 1 and 2 used for the search of the BS states.

Formula for the estimation of di- $\left(y_{0}\right)$ and tetraradicaloid $\left(y_{1}\right)$ indexes ${ }^{97}$ :

$y_{i}=1-\frac{2 T_{i}}{1+T_{i}^{2}}$

where $T_{i}$ represents the orbital overlap between the corresponding orbital pairs, which is expressed in terms of the occupation numbers $\left(n_{i}\right)$ of the UHF natural orbitals (UNOs) as $T_{i}=\frac{n_{\mathrm{HONO}-i}-n_{\mathrm{LUNO}+i}}{2}$

Formula for the estimation of the effective number of unpaired electrons $\left(N_{U}\right)^{98}$ :

$N_{\mathrm{U}}=\sum_{i}\left(1-\operatorname{abs}\left(1-n_{i}\right)\right)$

where $\left(n_{i}\right)$ are natural occupation numbers. 


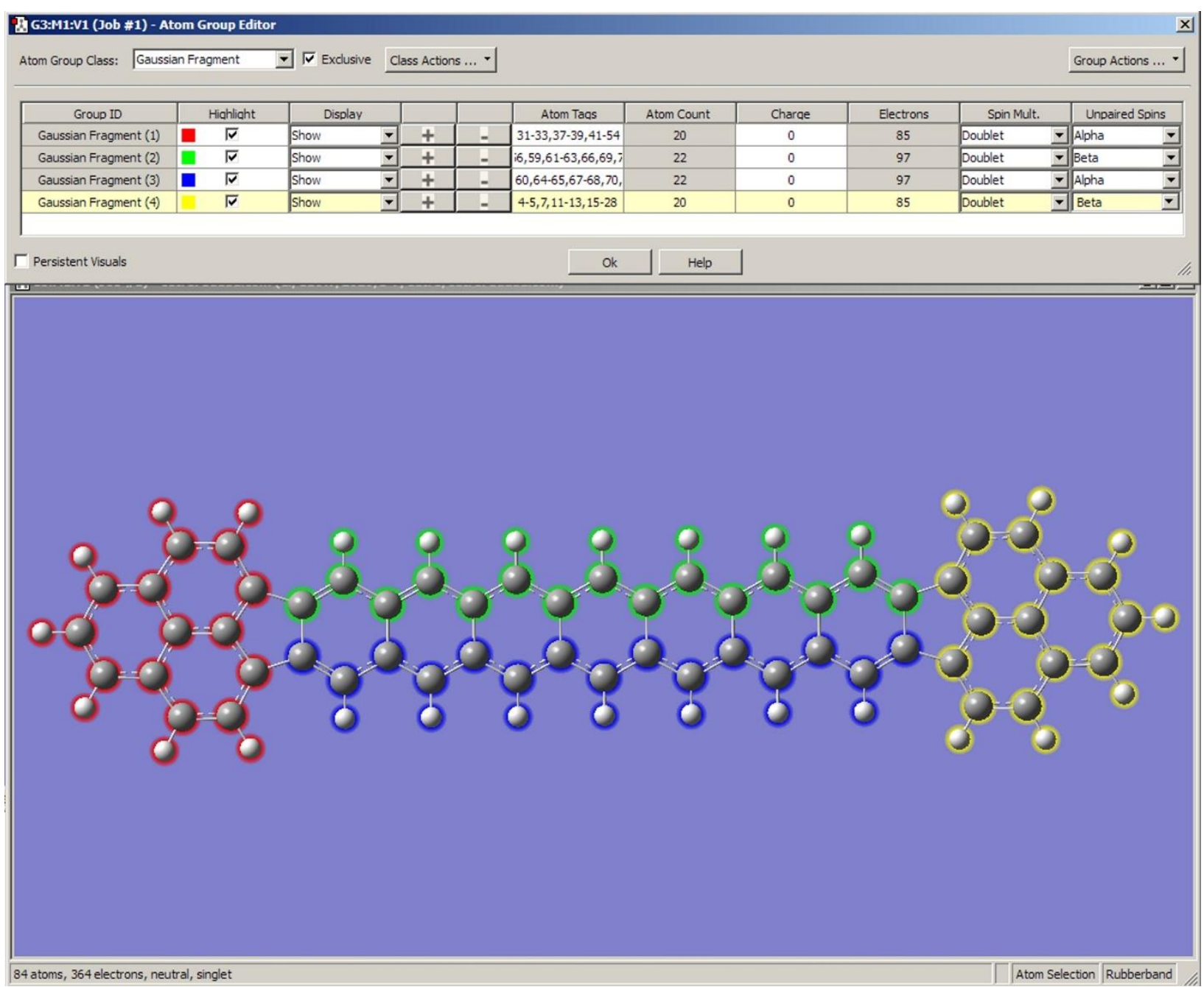

Figure S1. An example of partitioning into the fragments of a molecule with the use of Gauss View 6.0 program.

\section{A sample of input for calculating the BS state:}

$\%$ chk=cetre7abab_sample

$\%$ mem $=2700 \mathrm{MW}$

\#p ub3lyp/6-311++g(d,p) guess=fragment=4

sample input for generation BS state

$01020-2020-2$

$\begin{array}{llll}\mathrm{C}(\text { Fragment=2) } & 8.56323700 & 0.73709200 & 0.00007600 \\ \mathrm{C}(\text { Fragment=3) } & 6.14406500 & -0.74804400 & -0.00010600 \\ \mathrm{C}(\text { Fragment=2) } & 6.13502000 & 0.70840700 & -0.00008200 \\ \mathrm{C}(\text { Fragment=4) } & 12.13736300 & 0.02820500 & 0.00024300\end{array}$


$\begin{array}{lcrc}\mathrm{C}(\text { Fragment=4) } & 10.74278300 & 0.02162400 & 0.00014500 \\ \mathrm{C}(\text { Fragment=3) } & 8.57405800 & -0.73329500 & 0.00004400 \\ \mathrm{C}(\text { Fragment=4) } & 9.96070600 & -1.16203700 & 0.00018100\end{array}$

$\mathrm{H}($ Fragment=1) $\quad-12.51165900 \quad 3.42861000 \quad 0.00058100$

--link1--

$\%$ chk=cetre7abab_sample

$\%$ mem $=2700 \mathrm{MW}$

\#p opt=loose ub3lyp/6-311++g(d,p) guess=read geom=check

geometry optimization

01

--link1--

$\%$ chk=cetre7abab_sample

$\%$ mem $=2700 \mathrm{MW}$

\#p stable ub3lyp/6-311++g(d,p) guess=read geom=check

checking for stabilities of DFT WF

01 


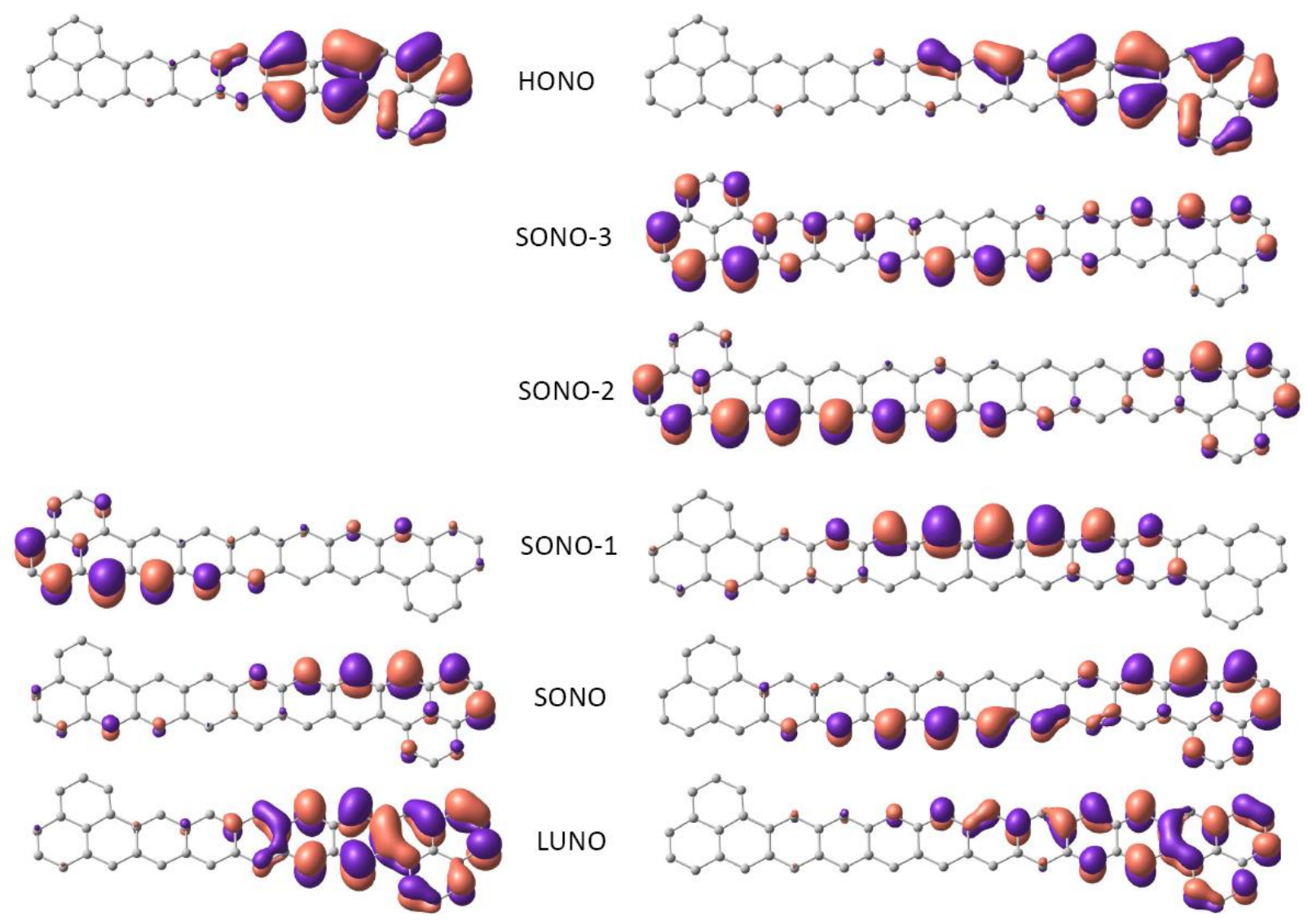

Figure S2. Natural orbitals of the compounds $\mathbf{1}(n=1)(\mathrm{left})$ and $\mathbf{1}(n=4)($ right $)$, cutoff $=0.03$ e $\AA^{-3}$.

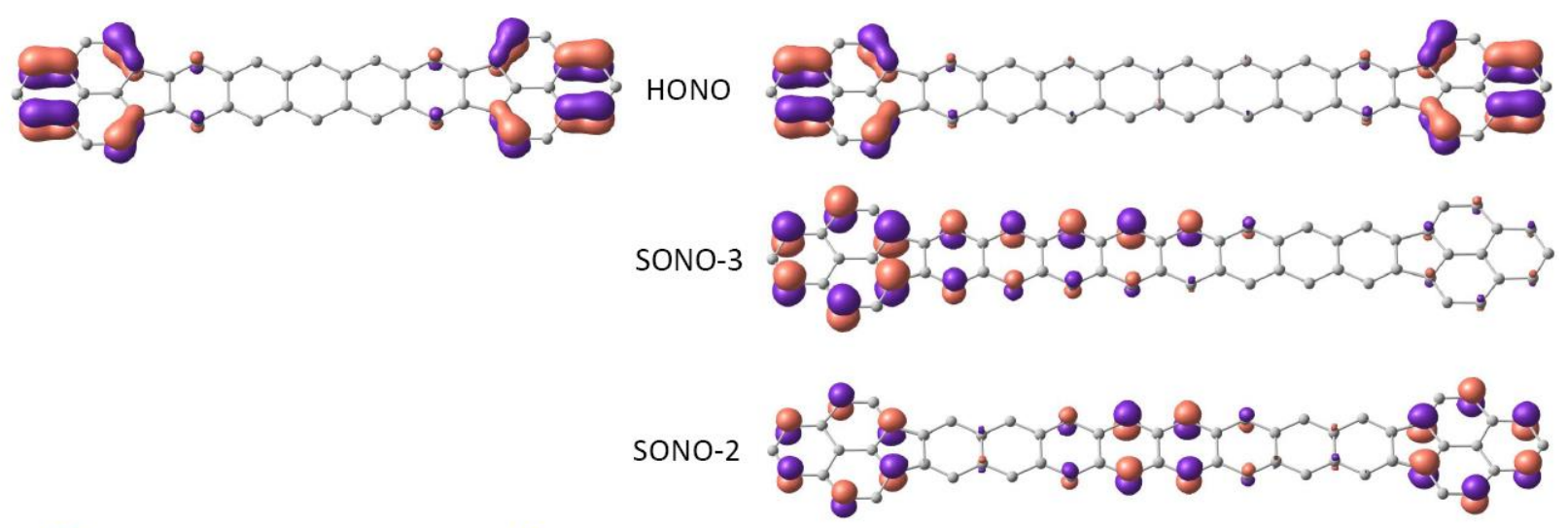

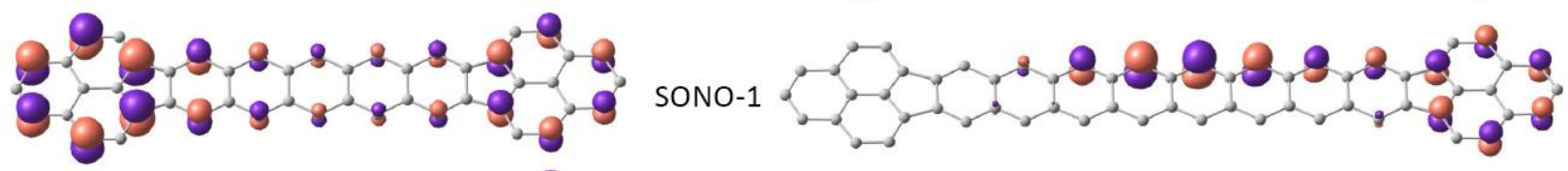

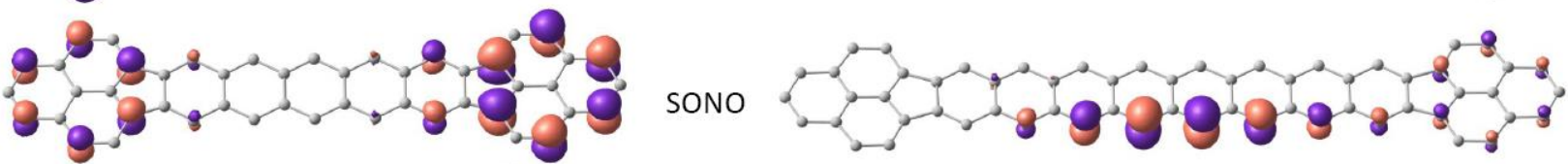

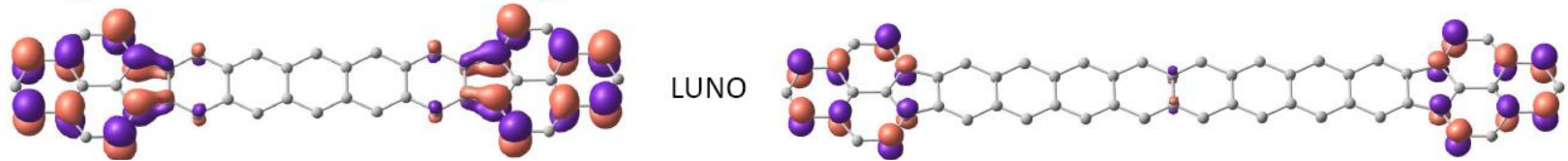

Figure S2. Natural orbitals of the compounds $2(n=1)($ left $)$ and $2(n=4)$ (right), cutoff $=0.03$ e $\AA^{-3}$. 
Table S1. Multiplicity $\left(M_{\mathrm{s}}\right)$, total energies without $\left(E_{\text {total }}\right.$, a.u. $)$ and with taking into account for

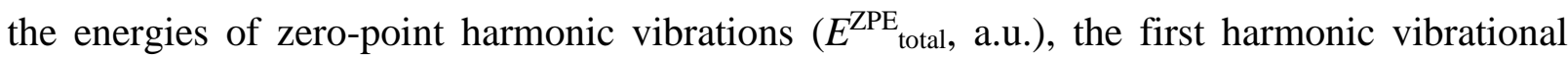
frequencies $\left(\omega_{1}, \mathrm{~cm}^{-1}\right)$ and expectation values of the spin-squared operator $\left(\hat{\mathrm{S}}^{2}\right)$ of the structures of compounds 1 calculated by the DFT UB3LYP/6-311++G(d,p) method.

\begin{tabular}{|c|c|c|c|c|c|}
\hline Structure & $M_{\mathrm{s}}$ & $E_{\text {total }}$ & $E^{\mathrm{ZPE}} \mathrm{total}_{\text {tol }}$ & $\omega_{1}$ & $\hat{\mathrm{S}}^{2}$ \\
\hline $\operatorname{css} 1(n=1)$ & 1 & -1691.583189 & -1691.054104 & 12 & 0.000 \\
\hline $\operatorname{oss} 1(n=1)$ & 1 & -1691.592205 & - & - & 1.228 \\
\hline $\mathrm{T} \mathbf{1}(n=1)$ & 3 & -1691.585555 & -1691.058687 & 11 & 2.116 \\
\hline $\mathrm{Q} \mathbf{1}(n=1)$ & 5 & -1691.558482 & -1691.033564 & 11 & 6.126 \\
\hline $\operatorname{css} 1(n=2)$ & 1 & -1845.250724 & -1844.675581 & 9 & 0.000 \\
\hline $\operatorname{oss} 1(n=2)$ & 1 & -1845.262060 & - & - & 1.435 \\
\hline${ }_{\mathrm{T}} \mathbf{1}(n=2)$ & 3 & -1845.254879 & -1844.682411 & 9 & 2.158 \\
\hline $\mathrm{Q} \mathbf{1}(n=2)$ & 5 & -1845.234900 & -1844.663794 & 9 & 6.008 \\
\hline $\operatorname{css} 1(n=3)$ & 1 & -1998.918230 & -1998.296934 & 7 & 0.000 \\
\hline oss $1(n=3)$ & 1 & -1998.931917 & - & - & 1.646 \\
\hline $\mathrm{T} \mathbf{1}(n=3)$ & 3 & -1998.923849 & -1998.306100 & 8 & 2.351 \\
\hline $\mathrm{Q} \mathbf{1}(n=3)$ & 5 & -1998.909400 & -1998.292136 & 8 & 6.150 \\
\hline $\mathrm{Q} \mathbf{1}(n=4)$ & 5 & -2152.582533 & -2151.919192 & 7 & 6.170 \\
\hline$\beta \alpha \alpha \alpha$ & 3 & -2152.586076 & - & - & 2.727 \\
\hline$\alpha \beta \alpha \alpha$ & 3 & -2152.592789 & - & - & 2.342 \\
\hline$\beta \beta \alpha \alpha$ & 1 & -2152.602021 & - & - & 1.886 \\
\hline$\alpha \beta \beta \alpha$ & 1 & -2152.590414 & - & - & 1.463 \\
\hline $\mathrm{Q} \mathbf{1}(n=5)$ & 5 & -2306.254614 & -2305.545279 & 6 & 6.199 \\
\hline$\beta \alpha \alpha \alpha$ & 3 & -2306.256829 & - & - & 2.921 \\
\hline$\alpha \beta \alpha \alpha$ & 3 & -2306.263700 & - & - & 2.988 \\
\hline$\beta \beta \alpha \alpha$ & 1 & -2306.272162 & - & - & 2.100 \\
\hline$\alpha \beta \beta \alpha$ & 1 & -2306.260496 & - & - & 1.666 \\
\hline
\end{tabular}


Table S2. Multiplicity $\left(M_{\mathrm{s}}\right)$, total energies without $\left(E_{\text {total }}\right.$, a.u. $)$ and with taking into account for

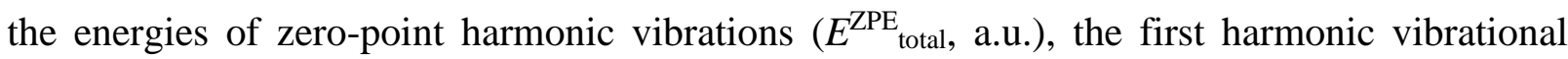
frequencies $\left(\omega_{1}, \mathrm{~cm}^{-1}\right)$ and expectation values of the spin-squared operator $\left(\hat{\mathrm{S}}^{2}\right)$ of the structures of compounds 2 calculated by the DFT UB3LYP/6-311++G(d,p) method.

\begin{tabular}{|c|c|c|c|c|c|}
\hline Structure & $M_{\mathrm{s}}$ & $E_{\text {total }}$ & $E^{\mathrm{ZPE}}{ }_{\text {total }}$ & $\omega_{1}$ & $\hat{\mathrm{S}}^{2}$ \\
\hline $\operatorname{css} 2(n=1)$ & 1 & -1844.048199 & -1843.495300 & 9 & 0.000 \\
\hline $\operatorname{oss} 2(n=1)$ & 1 & -1844.060464 & - & - & 1.037 \\
\hline $\mathrm{T} 2(n=1)$ & 3 & -1844.059598 & -1843.507615 & 10 & 2.079 \\
\hline $\mathrm{Q} 2(n=1)$ & 5 & -1844.021258 & -1843.471644 & 10 & 6.095 \\
\hline $\operatorname{css} 2(n=2)$ & 1 & -1997.713908 & -1997.115266 & 9 & 0.000 \\
\hline oss $2(n=2)$ & 1 & -1997.728280 & - & - & 6.100 \\
\hline${ }_{\mathrm{T}} 2(n=2)$ & 3 & -1997.727750 & -1997.130081 & 8 & 1.053 \\
\hline $\mathrm{Q} 2(n=2)$ & 5 & -1997.701562 & -1997.105595 & 9 & 2.081 \\
\hline $\mathrm{Q} 2(n=3)$ & 5 & -2151.378523 & -2150.736270 & 7 & 6.105 \\
\hline$\beta \alpha \alpha \alpha$ & 3 & -2151.382747 & - & - & 2.852 \\
\hline$\alpha \beta \alpha \alpha$ & 3 & -2151.395959 & - & - & 2.465 \\
\hline$\beta \beta \alpha \alpha$ & 1 & -2151.396095 & - & - & 1.406 \\
\hline$\alpha \beta \beta \alpha$ & 1 & -2151.385955 & - & - & 1.761 \\
\hline $\mathrm{Q} 2(n=4)$ & 5 & -2305.052859 & -2304.364570 & 8 & 6.109 \\
\hline$\beta \alpha \alpha \alpha$ & 3 & -2305.056168 & - & - & 2.931 \\
\hline$\alpha \beta \alpha \alpha$ & 3 & -2305.065657 & - & - & 2.828 \\
\hline$\beta \beta \alpha \alpha$ & 1 & -2305.065637 & - & - & 1.821 \\
\hline$\alpha \beta \beta \alpha$ & 1 & -2305.058505 & - & - & 1.843 \\
\hline $\mathrm{Q} 2(n=5)$ & 5 & -2458.725600 & -2457.991120 & 6 & 6.113 \\
\hline$\beta \alpha \alpha \alpha$ & 3 & -2458.727978 & - & - & 2.979 \\
\hline$\alpha \beta \alpha \alpha$ & 3 & -2458.735764 & - & - & 3.097 \\
\hline$\beta \beta \alpha \alpha$ & 1 & -2458.735731 & - & - & 2.099 \\
\hline$\alpha \beta \beta \alpha$ & 1 & -2458.729815 & - & - & 1.899 \\
\hline
\end{tabular}




\section{Cartesian coordinates of the structures of compounds 1 and 2}

calculated by the DFT UB3LYP/6-311++G(d,p) method

$\begin{array}{lccc}\text { CsS1 } & (n=1) & & \\ 6 & -6.189238000 & -0.308790000 & -0.000121000 \\ 6 & 1.278724000 & 0.639386000 & -0.000003000 \\ 6 & 1.173184000 & -0.819895000 & 0.000067000 \\ 6 & -8.682255000 & -0.053926000 & -0.000188000 \\ 6 & -7.538008000 & -0.907670000 & -0.000117000 \\ 6 & -6.080247000 & 1.151364000 & -0.000194000 \\ 6 & 2.582085000 & 1.221443000 & -0.000002000 \\ 1 & 2.661420000 & 2.304522000 & -0.000053000 \\ 6 & 0.127364000 & 1.401076000 & -0.000066000 \\ 6 & -7.219654000 & 1.929922000 & -0.000260000 \\ 1 & -7.119898000 & 3.010932000 & -0.000313000 \\ 6 & -8.531582000 & 1.373614000 & -0.000260000 \\ 6 & -0.127383000 & -1.401288000 & 0.000066000 \\ 6 & -9.997768000 & -0.612364000 & -0.000186000 \\ 6 & -11.122906000 & 0.256632000 & -0.000257000 \\ 1 & -12.117003000 & -0.176975000 & -0.000256000 \\ 6 & -7.748188000 & -2.286238000 & -0.000047000 \\ 6 & -10.148643000 & -2.018612000 & -0.000114000 \\ 1 & -11.146912000 & -2.442695000 & -0.000113000 \\ 6 & -10.956963000 & 1.625012000 & -0.000327000 \\ 1 & -11.823462000 & 2.276765000 & -0.000381000 \\ 6 & -9.670709000 & 2.183293000 & -0.000328000 \\ 1 & -9.550173000 & 3.261362000 & -0.000382000 \\ 6 & -9.038196000 & -2.834886000 & -0.000045000 \\ 1 & -9.155746000 & -3.912765000 & 0.000011000 \\ 6 & 6.189227000 & 0.308773000 & 0.000121000 \\ 6 & 3.623977000 & -0.995703000 & 0.000131000 \\ 6 & 3.728785000 & 0.459153000 & 0.000061000 \\ 6 & 8.682247000 & 0.054020000 & 0.000188000 \\ 6 & 7.537965000 & 0.907715000 & 0.000117000 \\ 6 & 6.080281000 & -1.151375000 & 0.000194000 \\ 6 & 2.326894000 & -1.581480000 & 0.000131000 \\ 1 & 2.248892000 & -2.664522000 & 0.000182000 \\ 6 & 4.785198000 & -1.743315000 & 0.000195000 \\ 6 & 7.219732000 & -1.929891000 & 0.000260000 \\ 1 & 7.120012000 & -3.010906000 & 0.000313000 \\ 6 & 8.531626000 & -1.373532000 & 0.000260000 \\ 6 & 5.036392000 & 1.046001000 & 0.000060000 \\ 6 & 9.997736000 & 0.612513000 & 0.000186000 \\ 1 & 11.122916000 & -0.256435000 & 0.000257000 \\ & 12.116992000 & 0.177220000 & 0.000256000\end{array}$




\begin{tabular}{|c|c|c|c|}
\hline & 7.748091000 & & \\
\hline & 10.148557000 & 2.018766000 & 0.000114000 \\
\hline & 11.146812000 & 2.442883000 & 0.000113000 \\
\hline & 10.957027000 & -1.624821000 & 0.000327000 \\
\hline & 11.823548000 & -2.276545000 & 0.00038100 \\
\hline & 9.670797000 & -2.183159000 & 0.000328000 \\
\hline & 9.550317000 & $-3.2612 ?$ & 82000 \\
\hline & 9.038 & 2.834997000 & 0.000045000 \\
\hline & 9.155581000 & 3.912881000 & -0.000010000 \\
\hline & 4.719922000 & -2.827160000 & 0.000247000 \\
\hline & 0.207277000 & 2.484026000 & 7000 \\
\hline & 6.90 & 2.96504 & -0.000 \\
\hline & -6.905147000 & -2.965018000 & 0.000009000 \\
\hline & -0.207203000 & -2.484246000 & 17000 \\
\hline & 5.077861000 & 2.128826000 & 0.000007000 \\
\hline & -3.62 & 0.9955 & 1000 \\
\hline & -3.72 & -0.459272000 & 61000 \\
\hline & -2.32 & 1.581310000 & 1000 \\
\hline & -2.58214 & -1.221599000 & 0.000002000 \\
\hline & -5.03 & -1.0460 & 50000 \\
\hline & -4.78 & 1.743245000 & -0.00 \\
\hline & -5.07 & -2.128882000 & -0.0 \\
\hline & -2.248 & 2.664346000 & -0.00 \\
\hline & -2.66 & -2.30468 & 0.0 \\
\hline & -4.71 & 2.82708 & -0.00 \\
\hline & -1.27 & -0.63958 & 3000 \\
\hline & -1.173145000 & 0.819715000 & -0.000067000 \\
\hline \multicolumn{4}{|c|}{$\mathrm{ss} \mathbf{1}(n=1)$} \\
\hline & -6.18521 & -0.298783000 & -0.0 \\
\hline & & 0.633293000 & -0.00 \\
\hline & 1.171160000 & -0.81920 & 0.00 \\
\hline & -8.67 & -0.05641 & -0.00 \\
\hline & -7.529 & -0.90317 & -0.0 \\
\hline & -6.07927 & 1.156177000 & -0.000195000 \\
\hline & 2.569639000 & 1.216176000 & -0.000002000 \\
\hline & 2.649 & 2.299168000 & -0.0000 \\
\hline & 0.113296000 & 1.401578000 & -0.000067000 \\
\hline & -7.237196000 & 1.938593000 & -0.00026100 \\
\hline & -7.141902000 & 3.019772000 & -0.00031400 \\
\hline & -8.537024000 & 1.374638000 & -0.000261000 \\
\hline & -0.113294000 & -1.401770000 & 0.00006700 \\
\hline & -9.989823000 & -0.623158000 & -0.00018600 \\
\hline & -11.121955000 & 0.238888000 & -0.00025600 \\
\hline & -12.112684000 & -0.202387000 & -0.0002550 \\
\hline & -7.731855000 & -2.285307000 & -0.00004800 \\
\hline
\end{tabular}




\begin{tabular}{|c|c|c|c|}
\hline 6 & -10.133062000 & -2.029249000 & -0.000113000 \\
\hline 1 & -11.129074000 & -2.458708000 & -0.000112000 \\
\hline 6 & -10.966660000 & 1.610450000 & -0.000326000 \\
\hline 1 & -11.838560000 & 2.254964000 & -0.000379000 \\
\hline 6 & -9.687135000 & 2.178476000 & -0.000328000 \\
\hline 1 & -9.574442000 & 3.257302000 & -0.000382000 \\
\hline 6 & -9.017381000 & -2.840496000 & -0.000045000 \\
\hline 1 & -9.129526000 & -3.918967000 & 0.000010000 \\
\hline 6 & 6.185209000 & 0.298790000 & 0.000122000 \\
\hline 6 & 3.621377000 & -0.999342000 & 0.000132000 \\
\hline 6 & 3.726538000 & 0.449391000 & 0.000062000 \\
\hline 6 & 8.677880000 & 0.056498000 & 0.000188000 \\
\hline 6 & 7.529298000 & 0.903229000 & 0.000118000 \\
\hline 6 & 6.079305000 & -1.156151000 & 0.000195000 \\
\hline 6 & 2.342512000 & -1.587556000 & 0.000132000 \\
\hline 1 & 2.265151000 & -2.670554000 & 0.000184000 \\
\hline 6 & 4.802164000 & -1.751816000 & 0.000196000 \\
\hline 6 & 7.237242000 & -1.938536000 & 0.000261000 \\
\hline 1 & 7.141921000 & -3.019712000 & 0.000314000 \\
\hline 6 & 8.537035000 & -1.374564000 & 0.000261000 \\
\hline 6 & 5.026295000 & 1.037789000 & 0.000060000 \\
\hline 6 & 9.989820000 & 0.623255000 & 0.000186000 \\
\hline 6 & 11.121967000 & -0.238764000 & 0.000256000 \\
\hline 1 & 12.112689000 & 0.202519000 & 0.000254000 \\
\hline 6 & 7.731817000 & 2.285376000 & 0.000048000 \\
\hline 6 & 10.133027000 & 2.029347000 & 0.000113000 \\
\hline 1 & 11.129028000 & 2.458829000 & 0.000112000 \\
\hline 6 & 10.966685000 & -1.610331000 & 0.000326000 \\
\hline 1 & 11.838597000 & -2.254830000 & 0.000379000 \\
\hline 6 & 9.687182000 & -2.178370000 & 0.000328000 \\
\hline 1 & 9.574497000 & -3.257198000 & 0.000382000 \\
\hline 6 & 9.017328000 & 2.840573000 & 0.000046000 \\
\hline 1 & 9.129465000 & 3.919046000 & -0.000010000 \\
\hline 1 & 4.739045000 & -2.835671000 & 0.000249000 \\
\hline 1 & 0.194027000 & 2.484414000 & -0.000119000 \\
\hline 1 & 6.885123000 & 2.959195000 & -0.000007000 \\
\hline 1 & -6.885161000 & -2.959133000 & 0.000008000 \\
\hline 1 & -0.193853000 & -2.484614000 & 0.000119000 \\
\hline 1 & 5.067857000 & 2.120396000 & 0.000008000 \\
\hline 6 & -3.621353000 & 0.999267000 & -0.000132000 \\
\hline 6 & -3.726566000 & -0.449487000 & -0.000061000 \\
\hline 6 & -2.342482000 & 1.587379000 & -0.000132000 \\
\hline 6 & -2.569668000 & -1.216292000 & 0.000002000 \\
\hline 6 & -5.026353000 & -1.037805000 & -0.000060000 \\
\hline 6 & -4.802117000 & 1.751767000 & -0.000196000 \\
\hline 1 & -5.067914000 & -2.120428000 & -0.000007000 \\
\hline
\end{tabular}




$\begin{array}{rrrr}1 & -2.264993000 & 2.670381000 & -0.000184000 \\ 1 & -2.649934000 & -2.299294000 & 0.000054000 \\ 1 & -4.738961000 & 2.835625000 & -0.000250000 \\ 6 & -1.278922000 & -0.633435000 & 0.000003000 \\ 6 & -1.171178000 & 0.819067000 & -0.000068000\end{array}$

$\begin{array}{lccc}\mathrm{T} \mathbf{1} & (n=1) & & \\ 6 & -6.179827000 & -0.297867000 & -0.000488000 \\ 6 & 1.277328000 & 0.630917000 & -0.000138000 \\ 6 & 1.166648000 & -0.819057000 & 0.000054000 \\ 6 & -8.673976000 & -0.058464000 & -0.000628000 \\ 6 & -7.525158000 & -0.902835000 & -0.000400000 \\ 6 & -6.073008000 & 1.160600000 & -0.000783000 \\ 6 & 2.567159000 & 1.214299000 & -0.000080000 \\ 1 & 2.648430000 & 2.297096000 & -0.000208000 \\ 6 & 0.108712000 & 1.401723000 & -0.000364000 \\ 6 & -7.243006000 & 1.942387000 & -0.001016000 \\ 1 & -7.147208000 & 3.023441000 & -0.001251000 \\ 6 & -8.534795000 & 1.375695000 & -0.000952000 \\ 6 & -0.108709000 & -1.401724000 & 0.000035000 \\ 6 & -9.984947000 & -0.626792000 & -0.000535000 \\ 6 & -11.119450000 & 0.232720000 & -0.000769000 \\ 1 & -12.109138000 & -0.210640000 & -0.000693000 \\ 6 & -7.724654000 & -2.285270000 & -0.000085000 \\ 6 & -10.125843000 & -2.032905000 & -0.000211000 \\ 1 & -11.121093000 & -2.464025000 & -0.000137000 \\ 6 & -10.966997000 & 1.605740000 & -0.001089000 \\ 1 & -11.840997000 & 2.247516000 & -0.001269000 \\ 6 & -9.690889000 & 2.176695000 & -0.001181000 \\ 1 & -9.580018000 & 3.255707000 & -0.001427000 \\ 6 & -9.008925000 & -2.842607000 & 0.000008000 \\ 1 & -9.119465000 & -3.921241000 & 0.000254000 \\ 6 & 6.179829000 & 0.297868000 & 0.000454000 \\ 6 & 3.613423000 & -1.002829000 & 0.000308000 \\ 6 & 3.721292000 & 0.446891000 & 0.000145000 \\ 6 & 8.673976000 & 0.058464000 & 0.000800000 \\ 6 & 7.525159000 & 0.902835000 & 0.000568000 \\ 6 & 6.073009000 & -1.160601000 & 0.000590000 \\ 6 & 2.349544000 & -1.591002000 & 0.000260000 \\ 1 & 2.268555000 & -2.673600000 & 0.000390000 \\ 6 & 4.809622000 & -1.757374000 & 0.000514000 \\ 6 & 7.243004000 & -1.942385000 & 0.000797000 \\ 1 & 7.147208000 & -3.023439000 & 0.000883000 \\ 6 & 8.534795000 & -1.375693000 & 0.000908000 \\ 6 & 5.023728000 & 1.035808000 & 0.000231000 \\ 6 & 9.984947000 & 0.626792000 & 0.000928000\end{array}$




\begin{tabular}{|c|c|c|c|}
\hline U & 11.119450000 & -0.232720000 & \\
\hline & 12.109139000 & 0.210639000 & 0.001260000 \\
\hline & 7.724652000 & 2.285269000 & 0.000463000 \\
\hline & 10.125843000 & 2.032906000 & 0.000820000 \\
\hline & 11.121092000 & 2.464027000 & 0.000923000 \\
\hline & 10.966996000 & -1.605740000 & \\
\hline & 11.840996000 & -2.24 & 28000 \\
\hline & 9.690887000 & -2.176 & 28000 \\
\hline & 9.580015000 & -3.255705000 & 0.001202000 \\
\hline & 9.008 & 2.842607000 & 39000 \\
\hline & 52000 & 3.9212 & 502000 \\
\hline & 4.743 & -2.840930000 & 1000 \\
\hline & 0.192098000 & 2.484228000 & -0.000513000 \\
\hline & 8000 & 2.957368000 & 2000 \\
\hline & 1000 & -2.9573 & 0.000099000 \\
\hline & -0.19 & -2.484229000 & 0.000185000 \\
\hline & 5.06 & 2.11 & 9000 \\
\hline & -3.6 & 1.0028260 & 5000 \\
\hline & -3.721292000 & -0.446890000 & -0.000 \\
\hline & -2.34 & 1.591001000 & -0.00 \\
\hline & -2.5 & -1.2142 & -0.00 \\
\hline & -5.0 & -1.0358 & -0.0 \\
\hline & 22000 & 1.757374000 & -0.0008 \\
\hline & -5.06 & -2.118349000 & -0.0 \\
\hline & -2.26 & 598000 & -0.0007 \\
\hline & -2.6 & -2.297096000 & 8000 \\
\hline & -4.7 & 2.840929000 & -0.00 \\
\hline & $-1.2^{\prime}$ & -0.6309 & 8000 \\
\hline & -1.166 & 0.819055000 & -0.0 \\
\hline \multicolumn{4}{|c|}{$n=1)$} \\
\hline & -6.1798 & -0.297867000 & -0.00 \\
\hline 6 & 1.27 & 0.630917000 & -0.000138000 \\
\hline 6 & 1.1666 & -0.819057000 & 4000 \\
\hline & -8.673 & -0.058464000 & -0.000628000 \\
\hline & -7.525 & -0.902835000 & -0.000400000 \\
\hline & -6.07 & 1.160600000 & -0.000783000 \\
\hline 6 & 2.567159000 & 1.214299000 & -0.000080000 \\
\hline & 2.648430000 & 2.297096000 & -0.000208000 \\
\hline & 0.108712000 & 1.401723000 & -0.000364000 \\
\hline & -7.243006000 & 1.942387000 & -0.001016000 \\
\hline . & -7.147208000 & 3.023441000 & -0.001251000 \\
\hline 6 & -8.534795000 & 1.375695000 & -0.000952000 \\
\hline 6 & -0.108709000 & -1.401724000 & 0.000035000 \\
\hline & -9.984947000 & -0.626792000 & -0.000535000 \\
\hline & -11.119450000 & 0.232720000 & -0.00076900 \\
\hline
\end{tabular}




$\begin{array}{lccc}1 & -12.109138000 & -0.210640000 & -0.000693000 \\ 6 & -7.724654000 & -2.285270000 & -0.000085000 \\ 6 & -10.125843000 & -2.032905000 & -0.000211000 \\ 1 & -11.121093000 & -2.464025000 & -0.000137000 \\ 6 & -10.966997000 & 1.605740000 & -0.001089000 \\ 1 & -11.840997000 & 2.247516000 & -0.001269000 \\ 6 & -9.690889000 & 2.176695000 & -0.001181000 \\ 1 & -9.580018000 & 3.255707000 & -0.001427000 \\ 6 & -9.008925000 & -2.842607000 & 0.000008000 \\ 1 & -9.119465000 & -3.921241000 & 0.000254000 \\ 6 & 6.179829000 & 0.297868000 & 0.000454000 \\ 6 & 3.613423000 & -1.002829000 & 0.000308000 \\ 6 & 3.721292000 & 0.446891000 & 0.000145000 \\ 6 & 8.673976000 & 0.058464000 & 0.000800000 \\ 6 & 7.525159000 & 0.902835000 & 0.000568000 \\ 6 & 6.073009000 & -1.160601000 & 0.000590000 \\ 6 & 2.349544000 & -1.591002000 & 0.000260000 \\ 1 & 2.268555000 & -2.673600000 & 0.000390000 \\ 6 & 4.809622000 & -1.757374000 & 0.000514000 \\ 6 & 7.243004000 & -1.942385000 & 0.000797000 \\ 1 & 7.147208000 & -3.023439000 & 0.000883000 \\ 6 & 8.534795000 & -1.375693000 & 0.000908000 \\ 6 & 5.023728000 & 1.035808000 & 0.000231000 \\ 6 & 9.984947000 & 0.626792000 & 0.000928000 \\ 6 & 11.119450000 & -0.232720000 & 0.001159000 \\ 1 & 12.109139000 & 0.210639000 & 0.001260000 \\ 6 & 7.724652000 & 2.285269000 & 0.000463000 \\ 6 & 10.125843000 & 2.032906000 & 0.000820000 \\ 6 & -3.613424000 & 1.002826000 & -0.000605000 \\ 6 & -2.721292000 & -0.446890000 & -0.000353000 \\ 6 & -2.567155000 & -1.214299000 & -0.000157000 \\ 1 & 1.0 .966996000 & -1.605740000 & 0.001254000 \\ 6 & 11.840996000 & -2.247515000 & 0.001428000 \\ 1 & 9.690887000 & -2.176693000 & 0.001128000 \\ 6 & 9.580015000 & -3.255705000 & 0.001202000 \\ 1 & 9.119462000 & 3.921242000 & 0.000502000 \\ 1 & 4.743859000 & -2.840930000 & 0.000621000 \\ 6 & 0.192098000 & 2.484228000 & -0.000513000 \\ 6 & -6.876561000 & -2.957371000 & 0.000099000 \\ 6 & -1.035809000 & -0.000306000\end{array}$




$\begin{array}{rrrr}6 & -4.809622000 & 1.757374000 & -0.000826000 \\ 1 & -5.064305000 & -2.118349000 & -0.000107000 \\ 1 & -2.268556000 & 2.673598000 & -0.000789000 \\ 1 & -2.648428000 & -2.297096000 & 0.000018000 \\ 1 & -4.743859000 & 2.840929000 & -0.001034000 \\ 6 & -1.277332000 & -0.630916000 & -0.000168000 \\ 6 & -1.166652000 & 0.819055000 & -0.000389000\end{array}$

$\begin{array}{lccc}\text { CSS1 } & (n=2) & & \\ 6 & 7.412392000 & -0.302168000 & 0.000175000 \\ 6 & -2.498384000 & 0.577584000 & -0.000042000 \\ 6 & -2.409062000 & -0.883032000 & -0.000136000 \\ 6 & 9.908138000 & -0.075242000 & 0.000227000 \\ 6 & 8.754383000 & -0.916048000 & 0.000202000 \\ 6 & 7.319906000 & 1.159444000 & 0.000231000 \\ 6 & -3.795160000 & 1.174345000 & -0.000028000 \\ 1 & -3.861844000 & 2.258284000 & 0.000043000 \\ 6 & -1.338435000 & 1.326279000 & 0.000032000 \\ 6 & 8.468325000 & 1.925088000 & 0.000267000 \\ 1 & 8.380818000 & 3.007169000 & 0.000302000 \\ 6 & 9.773738000 & 1.353979000 & 0.000256000 \\ 6 & -1.117214000 & -1.479224000 & -0.000144000 \\ 6 & 11.217241000 & -0.648478000 & 0.000234000 \\ 6 & 12.352097000 & 0.207787000 & 0.000255000 \\ 1 & 13.341216000 & -0.237050000 & 0.000257000 \\ 6 & 8.948931000 & -2.296857000 & 0.000207000 \\ 6 & 11.352280000 & -2.056368000 & 0.000223000 \\ 1 & 12.345692000 & -2.491709000 & 0.000227000 \\ 6 & 12.201792000 & 1.577999000 & 0.000277000 \\ 1 & 13.075651000 & 2.219834000 & 0.000294000 \\ 6 & 10.921977000 & 2.150805000 & 0.000280000 \\ 1 & 10.813636000 & 3.230161000 & 0.000303000 \\ 6 & 10.232704000 & -2.860057000 & 0.000215000 \\ 1 & 10.338060000 & -3.939191000 & 0.000216000 \\ 6 & -7.412392000 & 0.302167000 & -0.000152000 \\ 6 & -4.861852000 & -1.031209000 & -0.000199000 \\ 6 & -4.950334000 & 0.425033000 & -0.000101000 \\ 6 & -9.908138000 & 0.075240000 & -0.000209000 \\ 6 & -8.754384000 & 0.916047000 & -0.000127000 \\ 6 & -7.319905000 & -1.159444000 & -0.000256000 \\ 6 & -3.572672000 & -1.631549000 & -0.000212000 \\ 1 & -3.506463000 & -2.715376000 & -0.000283000 \\ 6 & -6.032079000 & -1.765728000 & -0.000274000 \\ 6 & -8.468324000 & -1.925088000 & -0.000335000 \\ 1 & -8.380816000 & -3.007170000 & -0.000412000\end{array}$




$\begin{array}{cccc}6 & -9.773737000 & -1.353980000 & -0.000316000 \\ 6 & -6.251450000 & 1.026390000 & -0.000082000 \\ 6 & -11.217241000 & 0.648475000 & -0.000186000 \\ 6 & -12.352097000 & -0.207791000 & -0.000270000 \\ 1 & -13.341216000 & 0.237045000 & -0.000252000 \\ 6 & -8.948932000 & 2.296856000 & -0.000023000 \\ 6 & -11.352281000 & 2.056365000 & -0.000079000 \\ 1 & -12.345693000 & 2.491706000 & -0.000062000 \\ 6 & -12.201791000 & -1.578003000 & -0.000373000 \\ 1 & -13.075649000 & -2.219838000 & -0.000437000 \\ 6 & -10.921975000 & -2.150808000 & -0.000396000 \\ 1 & -10.813634000 & -3.230163000 & -0.000475000 \\ 6 & -10.232706000 & 2.860055000 & 0.000000000 \\ 1 & -10.338062000 & 3.939188000 & 0.000082000 \\ 1 & -5.978719000 & -2.850240000 & -0.000349000 \\ 1 & -1.405899000 & 2.410080000 & 0.000101000 \\ 1 & -8.098303000 & 2.966107000 & 0.000043000 \\ 1 & 8.098301000 & -2.966108000 & 0.000209000 \\ 1 & -1.049255000 & -2.562972000 & -0.000212000 \\ 1 & -6.280490000 & 2.109638000 & -0.000008000 \\ 6 & 2.409061000 & 0.883035000 & 0.000094000 \\ 6 & 2.498383000 & -0.577582000 & 0.000007000 \\ 6 & 3.572672000 & 1.631552000 & 0.000169000 \\ 1 & 3.506464000 & 2.715380000 & 0.000231000 \\ 6 & 1.117214000 & 1.479227000 & 0.000099000 \\ 6 & 1.338435000 & -1.326276000 & -0.000071000 \\ 6 & 4.950333000 & -0.425032000 & 0.000086000 \\ 6 & 4.861852000 & 1.031212000 & 0.000167000 \\ 6 & 3.795160000 & -1.174343000 & 0.000007000 \\ 1 & 3.861843000 & -2.258283000 & -0.000054000 \\ 6 & 6.251449000 & -1.026390000 & 0.000095000 \\ 6 & 6.032080000 & 1.765730000 & 0.000234000 \\ 1 & 6.280489000 & -2.109638000 & 0.000033000 \\ 1 & 1.049255000 & 2.562976000 & 0.000164000 \\ 1 & 1.405898000 & -2.410077000 & -0.000136000 \\ 1 & 5.978721000 & 2.850242000 & 0.000285000 \\ 6 & 0.044897000 & -0.730186000 & -0.000067000 \\ 6 & -0.044898000 & 0.730190000 & 0.000024000\end{array}$

$\begin{array}{lrrr}\text { oss } 1(n=2) & & \\ 6 & -7.409684000 & 0.291653000 & 0.000155000 \\ 6 & 2.499767000 & -0.569161000 & -0.000032000 \\ 6 & 2.406967000 & 0.885275000 & -0.000100000 \\ 6 & -9.904284000 & 0.078381000 & 0.000220000 \\ 6 & -8.745873000 & 0.911169000 & 0.000150000 \\ 6 & -7.319553000 & -1.164671000 & 0.000227000\end{array}$




$\begin{array}{cccc}6 & 3.783040000 & -1.166871000 & -0.000032000 \\ 1 & 3.850232000 & -2.250716000 & 0.000018000 \\ 6 & 1.324983000 & -1.324131000 & 0.000033000 \\ 6 & -8.487240000 & -1.932956000 & 0.000294000 \\ 1 & -8.401851000 & -3.015030000 & 0.000347000 \\ 6 & -9.779290000 & -1.354462000 & 0.000294000 \\ 6 & 1.131783000 & 1.483045000 & -0.000099000 \\ 6 & -11.209463000 & 0.660335000 & 0.000218000 \\ 6 & -12.351535000 & -0.188113000 & 0.000289000 \\ 1 & -13.337073000 & 0.264473000 & 0.000287000 \\ 6 & -8.931169000 & 2.295961000 & 0.000079000 \\ 6 & -11.335394000 & 2.068214000 & 0.000145000 \\ 1 & -12.325991000 & 2.509922000 & 0.000143000 \\ 6 & -12.211932000 & -1.561700000 & 0.000359000 \\ 1 & -13.091553000 & -2.195710000 & 0.000413000 \\ 6 & -10.939797000 & -2.144492000 & 0.000362000 \\ 1 & -10.838961000 & -3.224525000 & 0.000416000 \\ 6 & -10.209853000 & 2.866166000 & 0.000076000 \\ 1 & -10.309276000 & 3.945883000 & 0.000020000 \\ 6 & 7.409681000 & -0.291674000 & -0.000155000 \\ 6 & 4.859985000 & 1.036613000 & -0.000165000 \\ 6 & 4.949742000 & -0.413683000 & -0.000095000 \\ 6 & 9.904288000 & -0.078372000 & -0.000220000 \\ 6 & 8.745892000 & -0.911179000 & -0.000151000 \\ 6 & 7.319538000 & 1.164649000 & -0.000227000 \\ 6 & 3.588706000 & 1.639736000 & -0.000164000 \\ 1 & 3.520221000 & 2.723307000 & -0.000215000 \\ 6 & 6.049853000 & 1.774897000 & -0.000229000 \\ 6 & 8.487217000 & 1.932949000 & -0.000293000 \\ 1 & 8.401822000 & 3.015022000 & -0.000347000 \\ 6 & 9.779272000 & 1.354470000 & -0.000293000 \\ 6 & 6.241682000 & -1.017171000 & -0.000094000 \\ 6 & 11.209483000 & -0.660298000 & -0.000218000 \\ 6 & 12.351538000 & 0.188171000 & -0.000289000 \\ 1 & 13.337086000 & -0.264395000 & -0.000287000 \\ 6 & 8.931233000 & -2.295970000 & -0.000079000 \\ 6 & 11.335451000 & -2.068172000 & -0.000145000 \\ 1 & 12.326058000 & -2.509857000 & -0.000143000 \\ 6 & 12.211910000 & 1.561755000 & -0.000359000 \\ 1 & 13.091520000 & 2.195779000 & -0.000412000 \\ 6 & 10.939765000 & 2.144523000 & -0.000361000 \\ 6 & 10.209929000 & -2.866148000 & -0.000077000 \\ 6 & -39519518000 & -2.407684000 & 0.000083000\end{array}$




\begin{tabular}{|c|c|c|c|}
\hline & 8.076191000 & -2.959284000 & -0.000024000 \\
\hline & -8.076101000 & 2.959248000 & 0.000023000 \\
\hline & 1.061101000 & 2.566482000 & -0.000149000 \\
\hline & 6.269617000 & -2.100251000 & -0.000042000 \\
\hline 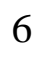 & -2.406977000 & -0.885293000 & 0.000100000 \\
\hline & -2.499779000 & 0.569143000 & 0.000031000 \\
\hline & -3.588719000 & -1.639758000 & 0.000165000 \\
\hline 1 & -3.520236000 & -2.723328000 & 0.000215000 \\
\hline 0 & -1.131795000 & -1.483061000 & 0.000099000 \\
\hline & -1.324998000 & 1.3241 & 033000 \\
\hline & -4.9497 & 0.413 & 0.000095000 \\
\hline 6 & -4.859996000 & -1.036634000 & 0.000165000 \\
\hline 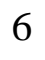 & -3.7830 & 1.16685 & 0.000032000 \\
\hline 1 & -3.85025 & 2.25069 & -0.000018000 \\
\hline & -6.24165 & 1.01715 & 0.000094000 \\
\hline 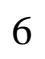 & $-6.04 \mathrm{C}$ & -1.7749 & 0.000 \\
\hline 1 & -6.2696 & $2.1002 ?$ & 42000 \\
\hline & -1.0611 & -2.5664 & 0.000149000 \\
\hline & -1.39 & 2.40 & -0.000083000 \\
\hline 1 & -5.995 & -2.8592 & 0.000281000 \\
\hline 6 & -0.04 & 0.72 & -0.00 \\
\hline & 0.047019000 & -0.727247000 & 0.000034000 \\
\hline \multicolumn{4}{|c|}{$\mathrm{T} \mathbf{1}(n=2)$} \\
\hline 6 & 7.4032 & -0.290945000 & 0.000146000 \\
\hline & -2.45 & & 025000 \\
\hline & -2.399 & -0.8871 & 08900( \\
\hline & 9.900 & -0.08 & 0.000217000 \\
\hline 0 & 8.7411 & -0.91212 & 134000 \\
\hline & & & \\
\hline & -3.78 & 1.16 & 28000 \\
\hline 1 & -3.849 & 2.24741 & 0.000 \\
\hline 6 & -1.3224 & 1.32309 & 0.000038000 \\
\hline 6 & 8.489419000 & 1.935543000 & 0.000295000 \\
\hline & 8.40823 & 3.01785 & 0.000350000 \\
\hline & 9.778 & 1.35 & 7000 \\
\hline 6 & -1.1360 & -1.484134000 & -0.000089000 \\
\hline 6 & 11.204469000 & -0.665007000 & 0.000217000 \\
\hline & 12.3487 & 0.1812 & 0.000299000 \\
\hline & 13.333167000 & -0.273799000 & 0.000301000 \\
\hline 6 & 8.924328000 & -2.296052000 & 0.000045000 \\
\hline 6 & 11.328672000 & -2.073042000 & 0.000132000 \\
\hline 1 & 12.318774000 & -2.515854000 & 0.00013600 \\
\hline & 12.212196000 & 1.554829000 & 0.000375000 \\
\hline 1 & 13.092943000 & 2.187260000 & 0.00043500 \\
\hline & 10.941053000 & 2.140404000 & 0.00037300 \\
\hline
\end{tabular}




\begin{tabular}{|c|c|c|c|}
\hline 1 & 10.843186000 & 3.220682000 & 0.000429000 \\
\hline & 10.202359000 & -2.868980000 & 0.000047000 \\
\hline 1 & 10.299476000 & -3.948894000 & -0.000020000 \\
\hline 6 & -7.403032000 & 0.290751000 & -0.000151000 \\
\hline 6 & -4.851180000 & -1.042605000 & -0.000151000 \\
\hline 6 & -4.941895000 & 0.410404000 & -0.000089000 \\
\hline 6 & -9.900204000 & 0.080088000 & -0.000225000 \\
\hline 6 & -8.741277000 & 0.911341000 & -0.000154000 \\
\hline 6 & -7.314042000 & -1.170298000 & -0.000215000 \\
\hline 6 & -3.594858000 & -1.645625000 & -0.000149000 \\
\hline 1 & -3.525910000 & -2.729005000 & -0.000196000 \\
\hline 6 & -6.055130000 & -1.782011000 & -0.000211000 \\
\hline 6 & -8.489913000 & -1.936462000 & -0.000279000 \\
\hline 1 & -8.407489000 & -3.018677000 & -0.000325000 \\
\hline 6 & -9.777707000 & -1.354704000 & -0.000288000 \\
\hline 6 & -6.238851000 & 1.014199000 & -0.000094000 \\
\hline 6 & -11.204415000 & 0.663574000 & -0.000232000 \\
\hline 6 & -12.348513000 & -0.182872000 & -0.000304000 \\
\hline 1 & -13.333089000 & 0.271756000 & -0.000310000 \\
\hline 6 & -8.924873000 & 2.295496000 & -0.000089000 \\
\hline 6 & -11.329029000 & 2.071475000 & -0.000167000 \\
\hline 1 & -12.319231000 & 2.514065000 & -0.000174000 \\
\hline 6 & -12.211626000 & -1.556848000 & -0.000365000 \\
\hline 1 & -13.092500000 & -2.189125000 & -0.000419000 \\
\hline 6 & -10.941021000 & -2.142145000 & -0.000357000 \\
\hline 1 & -10.842602000 & -3.222378000 & -0.000403000 \\
\hline 6 & -10.202933000 & 2.867888000 & -0.000096000 \\
\hline 1 & -10.300684000 & 3.947753000 & -0.000044000 \\
\hline 1 & -6.002063000 & -2.866278000 & -0.000256000 \\
\hline 1 & -1.393926000 & 2.406412000 & 0.000085000 \\
\hline 1 & -8.069095000 & 2.957836000 & -0.000030000 \\
\hline 1 & 8.068337000 & -2.958201000 & -0.000030000 \\
\hline 1 & -1.064235000 & -2.567380000 & -0.000138000 \\
\hline 1 & -6.266101000 & 2.097204000 & -0.000051000 \\
\hline 6 & 2.399112000 & 0.889286000 & 0.000107000 \\
\hline 6 & 2.493492000 & -0.564889000 & 0.000037000 \\
\hline 6 & 3.595017000 & 1.647280000 & 0.000168000 \\
\hline 1 & 3.526072000 & 2.730695000 & 0.000222000 \\
\hline 6 & 1.136811000 & 1.486262000 & 0.000108000 \\
\hline 6 & 1.323254000 & -1.321316000 & -0.000026000 \\
\hline 6 & 4.941680000 & -0.409892000 & 0.000095000 \\
\hline 6 & 4.851747000 & 1.044131000 & 0.000163000 \\
\hline 6 & 3.782038000 & -1.162546000 & 0.000033000 \\
\hline 1 & 3.849628000 & -2.246258000 & -0.000018000 \\
\hline 6 & 6.238956000 & -1.013779000 & 0.000092000 \\
\hline 6 & 6.054661000 & 1.782427000 & 0.000224000 \\
\hline
\end{tabular}




$\begin{array}{rrrc}1 & 6.265956000 & -2.096849000 & 0.000043000 \\ 1 & 1.064123000 & 2.569460000 & 0.000158000 \\ 1 & 1.393768000 & -2.404697000 & -0.000077000 \\ 1 & 6.002684000 & 2.866781000 & 0.000273000 \\ 6 & 0.047141000 & -0.724804000 & -0.000026000 \\ 6 & -0.048786000 & 0.726564000 & 0.000042000\end{array}$

$\begin{array}{lccc}\mathrm{Q} \mathbf{1} & (n=2) & & \\ 6 & -7.420165000 & -0.278335000 & -0.000197000 \\ 6 & 2.515459000 & 0.565784000 & 0.000088000 \\ 6 & 2.421405000 & -0.887852000 & 0.000242000 \\ 6 & -9.906209000 & -0.079932000 & -0.000267000 \\ 6 & -8.743138000 & -0.903389000 & -0.000293000 \\ 6 & -7.330736000 & 1.162678000 & -0.000216000 \\ 6 & 3.765841000 & 1.162610000 & 0.000035000 \\ 1 & 3.835822000 & 2.246305000 & -0.000080000 \\ 6 & 1.306779000 & 1.324444000 & -0.000008000 \\ 6 & -8.515694000 & 1.939291000 & -0.000224000 \\ 1 & -8.429450000 & 3.021106000 & -0.000218000 \\ 6 & -9.790103000 & 1.356463000 & -0.000215000 \\ 6 & 1.143957000 & -1.484922000 & 0.000282000 \\ 6 & -11.208186000 & -0.671179000 & -0.000320000 \\ 6 & -12.356003000 & 0.168731000 & -0.000280000 \\ 1 & -13.338297000 & -0.290736000 & -0.000315000 \\ 6 & -8.918891000 & -2.296912000 & -0.000429000 \\ 6 & -11.323887000 & -2.079153000 & -0.000421000 \\ 1 & -12.311246000 & -2.528031000 & -0.000460000 \\ 6 & -12.227789000 & 1.549409000 & -0.000206000 \\ 1 & -13.114715000 & 2.173380000 & -0.000172000 \\ 6 & -10.968311000 & 2.142266000 & -0.000179000 \\ 1 & -10.874498000 & 3.222836000 & -0.000134000 \\ 6 & -10.190689000 & -2.872185000 & -0.000488000 \\ 1 & -10.285327000 & -3.952426000 & -0.000591000 \\ 6 & 7.420165000 & 0.278333000 & 0.000150000 \\ 6 & 4.876487000 & -1.035011000 & 0.000288000 \\ 6 & 4.966854000 & 0.403999000 & 0.000129000 \\ 6 & 9.906209000 & 0.079946000 & 0.000163000 \\ 6 & 8.743132000 & 0.903395000 & 0.000077000 \\ 6 & 7.330744000 & -1.162685000 & 0.000315000 \\ 6 & 3.600443000 & -1.640590000 & 0.000340000 \\ 1 & 3.534765000 & -2.724311000 & 0.000459000 \\ 6 & 6.069115000 & -1.776372000 & 0.000378000 \\ 6 & 8.515708000 & -1.939289000 & 0.000404000 \\ 1 & 8.429472000 & -3.021105000 & 0.000527000 \\ 6 & 9.790113000 & -1.356450000 & 0.000331000 \\ 6 & 6.233217000 & 1.010050000 & 0.000065000\end{array}$




$\begin{array}{cccc}6 & 11.208181000 & 0.671204000 & 0.000084000 \\ 6 & 12.356005000 & -0.168698000 & 0.000172000 \\ 1 & 13.338294000 & 0.290776000 & 0.000111000 \\ 6 & 8.918873000 & 2.296919000 & -0.000080000 \\ 6 & 11.323872000 & 2.079179000 & -0.000079000 \\ 1 & 12.311227000 & 2.528065000 & -0.000140000 \\ 6 & 12.227800000 & -1.549378000 & 0.000335000 \\ 1 & 13.114730000 & -2.173342000 & 0.000401000 \\ 6 & 10.968327000 & -2.142244000 & 0.000415000 \\ 1 & 10.874521000 & -3.222814000 & 0.000540000 \\ 6 & 10.190667000 & 2.872204000 & -0.000157000 \\ 1 & 10.285297000 & 3.952445000 & -0.000279000 \\ 1 & 6.015364000 & -2.860709000 & 0.000494000 \\ 1 & 1.378391000 & 2.407862000 & -0.000115000 \\ 1 & 8.058929000 & 2.953348000 & -0.000144000 \\ 1 & -8.058956000 & -2.953349000 & -0.000513000 \\ 1 & 1.075193000 & -2.568452000 & 0.000394000 \\ 1 & 6.263393000 & 2.092684000 & -0.000057000 \\ 6 & -2.421404000 & 0.887824000 & -0.000050000 \\ 6 & -2.515461000 & -0.565802000 & 0.000078000 \\ 6 & -3.600438000 & 1.640565000 & -0.000144000 \\ 1 & -3.534757000 & 2.724282000 & -0.000230000 \\ 6 & -1.143958000 & 1.484895000 & -0.000073000 \\ 6 & -1.306783000 & -1.324464000 & 0.000195000 \\ 6 & -4.966854000 & -0.404008000 & -0.000041000 \\ 6 & -4.876481000 & 1.034991000 & -0.000134000 \\ 6 & -3.765843000 & -1.162620000 & 0.000079000 \\ 1 & -3.835826000 & -2.246312000 & 0.000167000 \\ 6 & -6.233219000 & -1.010052000 & -0.000073000 \\ 6 & -6.069105000 & 1.776357000 & -0.000199000 \\ 1 & -6.263397000 & -2.092682000 & 0.000013000 \\ 1 & -1.075194000 & 2.568425000 & -0.000171000 \\ 1 & -1.378397000 & -2.407880000 & 0.000295000 \\ 1 & -6.015348000 & 2.860693000 & -0.000225000 \\ 6 & -0.047836000 & -0.731081000 & 0.000174000 \\ 6 & 0.047832000 & 0.731056000 & 0.000029000\end{array}$

$\begin{array}{lccl}\operatorname{css} 1(n=3) \\ 6 & -8.636087000 & -0.302187000 & -0.000067000 \\ 6 & 3.720897000 & 0.535179000 & 0.000002000 \\ 6 & 3.643306000 & -0.926099000 & 0.000055000 \\ 6 & -11.132911000 & -0.096104000 & -0.000048000 \\ 6 & -9.972202000 & -0.926797000 & -0.000067000 \\ 6 & -8.554897000 & 1.160143000 & -0.000092000 \\ 6 & 5.011034000 & 1.143342000 & -0.000007000\end{array}$




\begin{tabular}{|c|c|c|c|}
\hline 1 & 5.067510000 & 2.227811000 & -0.000046000 \\
\hline & 2.553058000 & 1.273271000 & -0.000039000 \\
\hline 6 & -9.710042000 & 1.915540000 & -0.000092000 \\
\hline 1 & -9.629792000 & 2.998238000 & -0.000108000 \\
\hline 6 & -11.009621000 & 1.334348000 & -0.000061000 \\
\hline 6 & 2.358391000 & -1.533581000 & 0.000061000 \\
\hline 6 & -12.437231000 & -0.680100000 & -0.000029000 \\
\hline 6 & -13.579031000 & 0.166377000 & -0.000012000 \\
\hline 1 & -14.564497000 & -0.286391000 & 0.000006000 \\
\hline 6 & -10.154218000 & -2.309433000 & -0.000071000 \\
\hline 6 & -12.559731000 & -2.089298000 & -0.000030000 \\
\hline 1 & -13.549212000 & -2.533374000 & -0.000011000 \\
\hline 6 & -13.439833000 & 1.538086000 & -0.000019000 \\
\hline 1 & -14.319227000 & 2.172384000 & -0.000005000 \\
\hline 6 & -12.165414000 & 2.121336000 & -0.000046000 \\
\hline 1 & -12.065523000 & 3.201517000 & -0.000058000 \\
\hline 6 & -11.433054000 & -2.883347000 & -0.000051000 \\
\hline 1 & -11.529113000 & -3.963345000 & -0.000054000 \\
\hline 6 & 8.636073000 & 0.302153000 & 0.000050000 \\
\hline 6 & 6.096540000 & -1.052600000 & 0.000083000 \\
\hline 6 & 6.173644000 & 0.404288000 & 0.000031000 \\
\hline 6 & 11.132954000 & 0.096009000 & 0.000068000 \\
\hline 6 & 9.972270000 & 0.926752000 & 0.000039000 \\
\hline 6 & 8.554880000 & -1.160226000 & 0.000100000 \\
\hline 6 & 4.813613000 & -1.663430000 & 0.000091000 \\
\hline 1 & 4.754319000 & -2.747650000 & 0.000126000 \\
\hline 6 & 7.273018000 & -1.776545000 & 0.000116000 \\
\hline 6 & 9.710078000 & -1.915665000 & 0.000130000 \\
\hline 1 & 9.629917000 & -2.998361000 & 0.000163000 \\
\hline 6 & 11.009681000 & -1.334454000 & 0.000112000 \\
\hline 6 & 7.468812000 & 1.016661000 & 0.000018000 \\
\hline 6 & 12.437294000 & 0.679965000 & 0.000051000 \\
\hline 6 & 13.579084000 & -0.166554000 & 0.000077000 \\
\hline 1 & 14.564554000 & 0.286212000 & 0.000065000 \\
\hline 6 & 10.154382000 & 2.309354000 & -0.000004000 \\
\hline 6 & 12.559873000 & 2.089148000 & 0.000009000 \\
\hline 1 & 13.549376000 & 2.533182000 & -0.000004000 \\
\hline 6 & 13.439885000 & -1.538242000 & 0.000117000 \\
\hline 1 & 14.319260000 & -2.172564000 & 0.000138000 \\
\hline 6 & 12.165429000 & -2.121470000 & 0.000135000 \\
\hline 1 & 12.065539000 & -3.201651000 & 0.000168000 \\
\hline 6 & 11.433241000 & 2.883234000 & -0.000015000 \\
\hline 1 & 11.529337000 & 3.963230000 & -0.000045000 \\
\hline 1 & 7.226474000 & -2.861388000 & 0.000150000 \\
\hline 1 & 2.610762000 & 2.357599000 & -0.000076000 \\
\hline 1 & 9.297648000 & 2.970944000 & -0.000024000 \\
\hline
\end{tabular}




$\begin{array}{rrrr}1 & -9.297435000 & -2.970961000 & -0.000095000 \\ 1 & 2.297860000 & -2.617698000 & 0.000100000 \\ 1 & 7.488241000 & 2.100173000 & -0.000020000 \\ 6 & -3.643488000 & 0.926212000 & -0.000067000 \\ 6 & -3.720963000 & -0.535071000 & -0.000019000 \\ 6 & -4.813689000 & 1.663486000 & -0.000099000 \\ 1 & -4.754373000 & 2.747698000 & -0.000133000 \\ 6 & -2.358611000 & 1.533815000 & -0.000081000 \\ 6 & -2.553128000 & -1.273170000 & 0.000015000 \\ 6 & -6.173713000 & -0.404279000 & -0.000041000 \\ 6 & -6.096673000 & 1.052579000 & -0.000089000 \\ 6 & -5.010985000 & -1.143320000 & -0.000008000 \\ 1 & -5.067413000 & -2.227790000 & 0.000028000 \\ 6 & -7.468753000 & -1.016716000 & -0.000035000 \\ 6 & -7.272991000 & 1.776440000 & -0.000112000 \\ 1 & -7.488097000 & -2.100235000 & 0.000004000 \\ 1 & -2.298248000 & 2.617954000 & -0.000116000 \\ 1 & -2.610894000 & -2.357470000 & 0.000050000 \\ 1 & -7.226329000 & 2.861288000 & -0.000137000 \\ 6 & -1.266451000 & -0.665328000 & 0.000001000 \\ 6 & -1.188547000 & 0.796013000 & -0.000049000 \\ 6 & 1.266489000 & 0.665591000 & -0.000024000 \\ 6 & 1.188442000 & -0.795651000 & 0.000024000 \\ 6 & 0.096723000 & 1.403874000 & -0.000058000 \\ 6 & -0.096865000 & -1.403485000 & 0.000035000 \\ 1 & 0.155774000 & 2.488059000 & -0.000092000 \\ 1 & -0.156179000 & -2.487672000 & 0.000074000\end{array}$

$\begin{array}{lccc}\text { oss1 }(n=3) & & \\ 6 & -8.634925000 & 0.290494000 & 0.000043000 \\ 6 & 3.722332000 & -0.522543000 & -0.000013000 \\ 6 & 3.642173000 & 0.933634000 & -0.000045000 \\ 6 & -11.131009000 & 0.100887000 & 0.000060000 \\ 6 & -9.964599000 & 0.922688000 & 0.000036000 \\ 6 & -8.558137000 & -1.165825000 & 0.000076000 \\ 6 & 5.000440000 & -1.131121000 & -0.000005000 \\ 1 & 5.062017000 & -2.215129000 & 0.000018000 \\ 6 & 2.542735000 & -1.265627000 & 0.000011000 \\ 6 & -9.732453000 & -1.923197000 & 0.000099000 \\ 1 & -9.655614000 & -3.005839000 & 0.000122000 \\ 6 & -11.018843000 & -1.332875000 & 0.000093000 \\ 6 & 2.372827000 & 1.542296000 & -0.000051000 \\ 6 & -12.431112000 & 0.694650000 & 0.000052000 \\ 6 & -13.580633000 & -0.143274000 & 0.000076000 \\ 1 & -14.562298000 & 0.317521000 & 0.000071000 \\ 6 & -10.137157000 & 2.309368000 & 0.000004000\end{array}$




\begin{tabular}{|c|c|c|c|}
\hline & -12.543436000 & 2.103575000 & \\
\hline & -13.529503000 & 2.555217000 & \\
\hline & 13.453244000 & -1.518213000 & 8000 \\
\hline & -14.339057000 & -2.143630000 & \\
\hline & -12.186814000 & -2.112132000 & \\
\hline & -12.094556000 & -3.192974000 & \\
\hline & -11.4 & 2000 & \\
\hline & -11.500142000 & 3.971575000 & \\
\hline & 8.634905000 & -0.290505000 & -0.000042000 \\
\hline & 6.09 & & \\
\hline & 6.17 & -0.38 & \\
\hline & 11.1 & -0.10 & \\
\hline & 9.964577000 & -0.922 & -0.000 \\
\hline & 8.55 & & \\
\hline & 4.83 & 1.67 & 000 \\
\hline & 4.77 & 2.76 & \\
\hline & 7.29 & 1.78 & \\
\hline & 9.73 & & \\
\hline & 9.65 & 3.005 & 3000 \\
\hline & 11.0 & 1.3 & -0 . \\
\hline & 7.45 & -1.00 & -0.0 \\
\hline & 12.4 & -0.6 & \\
\hline & 629000 & 0.143 & -0.0 \\
\hline & 14.5 & -0.3 & -0.0 \\
\hline & 10.1 & -2.3 & 300 \\
\hline & 12.54 & -2.1 & \\
\hline & 13.52 & -2.555 & -0.0 \\
\hline & 13.4 & 1.5 & -0. \\
\hline & 14.3 & 2.1 & -0. \\
\hline & 12.1 & 2.11 & \\
\hline & 12.09 & 3.19 & -0.0 \\
\hline & 11.4 & -2.8 & \\
\hline & 11.5 & -3.9 & 0.0 \\
\hline & 7.25 & 2.87 & -0.0 \\
\hline & & -2.34 & \\
\hline & 9.27 & -2.96 & \\
\hline & -9.275 & 2.96 & -0.00 \\
\hline & 2.314 & 2.626 & -0.00 \\
\hline & 7.47925 & -2.088 & 4000 \\
\hline & -3.64 & -0.93 & 0.00004300 \\
\hline & -3.722333000 & 0.522604000 & 0.000010000 \\
\hline & -4.831112000 & -1.677148000 & 0.00006600 \\
\hline & -4.776096000 & -2.761319000 & 0.00008900 \\
\hline & -2.372818000 & -1.542241000 & 0.00004800 \\
\hline & -2.542718000 & 1.265693000 & -0.00001300 \\
\hline & -6.174037000 & 0.389206000 & 0.00002800 \\
\hline
\end{tabular}




$\begin{array}{rrrr}6 & -6.097493000 & -1.061898000 & 0.000061000 \\ 6 & -5.000427000 & 1.131180000 & 0.000005000 \\ 1 & -5.062016000 & 2.215187000 & -0.000019000 \\ 6 & -7.459370000 & 1.005109000 & 0.000021000 \\ 6 & -7.293757000 & -1.789133000 & 0.000084000 \\ 1 & -7.479280000 & 2.088186000 & -0.000002000 \\ 1 & -2.314114000 & -2.626325000 & 0.000072000 \\ 1 & -2.606461000 & 2.349531000 & -0.000036000 \\ 1 & -7.251297000 & -2.873830000 & 0.000108000 \\ 6 & -1.268668000 & 0.659242000 & -0.000008000 \\ 6 & -1.185984000 & -0.797097000 & 0.000025000 \\ 6 & 1.268697000 & -0.659165000 & 0.000005000 \\ 6 & 1.186018000 & 0.797159000 & -0.000026000 \\ 6 & 0.085262000 & -1.403872000 & 0.000030000 \\ 6 & -0.085262000 & 1.403933000 & -0.000031000 \\ 1 & 0.147252000 & -2.487861000 & 0.000053000 \\ 1 & -0.147234000 & 2.487923000 & -0.000055000\end{array}$

$\begin{array}{lccc}\mathrm{T} \mathbf{1} & (n=3) & & \\ 6 & 8.631401000 & 0.292085000 & -0.000049000 \\ 6 & -3.720541000 & -0.523133000 & 0.000017000 \\ 6 & -3.638018000 & 0.930253000 & 0.000057000 \\ 6 & 11.129192000 & 0.105677000 & -0.000064000 \\ 6 & 9.961573000 & 0.926267000 & -0.000050000 \\ 6 & 8.558141000 & -1.167600000 & -0.000078000 \\ 6 & -4.997957000 & -1.131561000 & 0.000005000 \\ 1 & -5.059483000 & -2.215660000 & -0.000025000 \\ 6 & -2.537152000 & -1.270606000 & -0.000010000 \\ 6 & 9.731743000 & -1.920600000 & -0.000096000 \\ 1 & 9.658855000 & -3.003720000 & -0.000115000 \\ 6 & 11.019546000 & -1.328048000 & -0.000086000 \\ 6 & -2.375741000 & 1.537314000 & 0.000067000 \\ 6 & 12.427990000 & 0.701499000 & -0.000060000 \\ 6 & 13.578975000 & -0.134740000 & -0.000074000 \\ 1 & 14.559669000 & 0.328272000 & -0.000069000 \\ 6 & 10.132180000 & 2.312359000 & -0.000040000 \\ 6 & 12.538815000 & 2.110783000 & -0.000045000 \\ 1 & 13.524608000 & 2.563137000 & -0.000041000 \\ 6 & 13.453723000 & -1.509036000 & -0.000093000 \\ 1 & 14.339629000 & -2.134195000 & -0.000103000 \\ 6 & 12.186934000 & -2.105137000 & -0.000100000 \\ 1 & 12.097756000 & -3.186226000 & -0.000116000 \\ 6 & 11.405107000 & 2.896374000 & -0.000037000 \\ 1 & 11.492937000 & 3.977088000 & -0.000028000 \\ 6 & -8.627861000 & -0.287877000 & 0.000041000 \\ 6 & -6.088436000 & 1.064493000 & 0.000072000\end{array}$




$\begin{array}{cccc}6 & -6.167160000 & -0.387147000 & 0.000030000 \\ 6 & -11.126278000 & -0.099545000 & 0.000051000 \\ 6 & -9.960683000 & -0.920259000 & 0.000025000 \\ 6 & -8.550469000 & 1.172766000 & 0.000084000 \\ 6 & -4.836928000 & 1.678316000 & 0.000084000 \\ 1 & -4.779903000 & 2.762379000 & 0.000115000 \\ 6 & -7.301240000 & 1.795278000 & 0.000098000 \\ 6 & -9.737466000 & 1.930656000 & 0.000110000 \\ 1 & -9.664087000 & 3.013403000 & 0.000141000 \\ 6 & -11.016381000 & 1.337544000 & 0.000095000 \\ 6 & -7.457412000 & -1.002577000 & 0.000017000 \\ 6 & -12.425525000 & -0.694354000 & 0.000035000 \\ 6 & -13.577259000 & 0.141943000 & 0.000062000 \\ 1 & -14.557785000 & -0.321338000 & 0.000049000 \\ 6 & -10.132126000 & -2.306431000 & -0.000016000 \\ 6 & -12.537864000 & -2.102938000 & -0.000008000 \\ 1 & -13.524020000 & -2.554442000 & -0.000020000 \\ 6 & -13.452975000 & 1.517968000 & 0.000104000 \\ 1 & -14.340117000 & 2.141452000 & 0.000124000 \\ 6 & -12.189085000 & 2.114781000 & 0.000120000 \\ 1 & -12.100138000 & 3.195814000 & 0.000153000 \\ 6 & -11.404620000 & -2.889751000 & -0.000032000 \\ 1 & -11.493339000 & -3.970400000 & -0.000065000 \\ 1 & -7.259128000 & 2.879959000 & 0.000130000 \\ 1 & -2.598820000 & -2.354636000 & -0.000039000 \\ 1 & -9.270388000 & -2.960997000 & -0.000036000 \\ 1 & 9.270376000 & 2.966733000 & -0.000036000 \\ 1 & -2.313391000 & 2.621260000 & 0.000097000 \\ 1 & -7.477725000 & -2.085616000 & -0.000015000 \\ 6 & 3.637300000 & -0.941858000 & -0.000038000 \\ 6 & 3.715419000 & 0.517620000 & 0.000000000 \\ 6 & 7.294225000 & -1.791735000 & -0.000083000 \\ 6 & 4.834599000 & -1.684749000 & -0.000065000 \\ 1 & 4.778719000 & -2.768887000 & -0.000092000 \\ 6 & 2.378762000 & -1.552501000 & -0.000045000 \\ 6 & 2.541407000 & 1.260217000 & 0.000029000 \\ 6 & 6.168838000 & 0.386728000 & -0.000022000 \\ 6 & 6.095364000 & -1.067923000 & -0.000059000 \\ 6 & 4.998924000 & 1.127159000 & 0.000007000 \\ 1 & 5.056270000 & 2.211452000 & 0.000034000 \\ 6 & 7.456883000 & 1.003716000 & -0.000018000 \\ 6 & 2.320536000 & -2.636484000 & -0.000072000 \\ 6 & 2.63057000 & 2.344199000 & 0.000057000 \\ 6 & -2.876688000 & -0.000105000 \\ 6 & 0.652396000 & 0.000022000\end{array}$




$\begin{array}{lrrc}6 & 1.179669000 & -0.805293000 & -0.000017000 \\ 6 & -1.273156000 & -0.664257000 & 0.000000000 \\ 6 & -1.190142000 & 0.790512000 & 0.000040000 \\ 6 & -0.078221000 & -1.413399000 & -0.000027000 \\ 6 & 0.083249000 & 1.397273000 & 0.000049000 \\ 1 & -0.137355000 & -2.497464000 & -0.000056000 \\ 1 & 0.147204000 & 2.481124000 & 0.000077000\end{array}$

$\begin{array}{lccc}\mathrm{Q} \mathbf{1}(n=3) & & \\ 6 & -8.641185000 & -0.280168000 & 0.000818000 \\ 6 & 3.737723000 & 0.515855000 & -0.001217000 \\ 6 & 3.657216000 & -0.935505000 & -0.001667000 \\ 6 & -11.132069000 & -0.103139000 & 0.001202000 \\ 6 & -9.962350000 & -0.917154000 & 0.002165000 \\ 6 & -8.566118000 & 1.166261000 & -0.000278000 \\ 6 & 4.986604000 & 1.125346000 & -0.000673000 \\ 1 & 5.045600000 & 2.209672000 & -0.000287000 \\ 6 & 2.523781000 & 1.264151000 & -0.001236000 \\ 6 & -9.757218000 & 1.929211000 & -0.000914000 \\ 1 & -9.682493000 & 3.011842000 & -0.001858000 \\ 6 & -11.028432000 & 1.334254000 & -0.000483000 \\ 6 & 2.382897000 & -1.544770000 & -0.001899000 \\ 6 & -12.428939000 & -0.704993000 & 0.002116000 \\ 6 & -13.584012000 & 0.125440000 & 0.001014000 \\ 1 & -14.562370000 & -0.342293000 & 0.001649000 \\ 6 & -10.126497000 & -2.309833000 & 0.004544000 \\ 6 & -12.533206000 & -2.113884000 & 0.004199000 \\ 1 & -13.516804000 & -2.570925000 & 0.004864000 \\ 6 & -13.467106000 & 1.505727000 & -0.000763000 \\ 1 & -14.358692000 & 2.122911000 & -0.001596000 \\ 6 & -12.210848000 & 2.109038000 & -0.001450000 \\ 1 & -12.126305000 & 3.190350000 & -0.002711000 \\ 6 & -11.394194000 & -2.896763000 & 0.005493000 \\ 1 & -11.479295000 & -3.977748000 & 0.007316000 \\ 6 & 8.641175000 & 0.280178000 & 0.000435000 \\ 6 & 6.109923000 & -1.061715000 & -0.001334000 \\ 6 & 6.185959000 & 0.379689000 & -0.000587000 \\ 6 & 11.132070000 & 0.103153000 & 0.000702000 \\ 6 & 9.962350000 & 0.917168000 & 0.001874000 \\ 6 & 8.566116000 & -1.166264000 & -0.000951000 \\ 6 & 4.843264000 & -1.678846000 & -0.001822000 \\ 1 & 4.786841000 & -2.763056000 & -0.002230000 \\ 6 & 7.312264000 & -1.791493000 & -0.001654000 \\ 6 & 9.757217000 & -1.929202000 & -0.001805000 \\ 1 & 9.682500000 & -3.011834000 & -0.002957000 \\ 6 & 11.028437000 & -1.334241000 & -0.001299000\end{array}$




$\begin{array}{cccc}6 & 7.453534000 & 0.998018000 & 0.000271000 \\ 6 & 12.428937000 & 0.705007000 & 0.001708000 \\ 6 & 13.584014000 & -0.125423000 & 0.000390000 \\ 1 & 14.562369000 & 0.342316000 & 0.001085000 \\ 6 & 10.126495000 & 2.309839000 & 0.004555000 \\ 6 & 12.533203000 & 2.113899000 & 0.004089000 \\ 1 & 13.516802000 & 2.570939000 & 0.004815000 \\ 6 & 13.467110000 & -1.505706000 & -0.001705000 \\ 1 & 14.358694000 & -2.122892000 & -0.002685000 \\ 6 & 12.210846000 & -2.109020000 & -0.002490000 \\ 1 & 12.126312000 & -3.190331000 & -0.003962000 \\ 6 & 11.394193000 & 2.896774000 & 0.005586000 \\ 1 & 11.479291000 & 3.977759000 & 0.007632000 \\ 1 & 7.269383000 & -2.876259000 & -0.002519000 \\ 1 & 2.586235000 & 2.348141000 & -0.001017000 \\ 1 & 9.261262000 & 2.959317000 & 0.006046000 \\ 1 & -9.261265000 & -2.959312000 & 0.005855000 \\ 1 & 2.323120000 & -2.628819000 & -0.002134000 \\ 1 & 7.472269000 & 2.080949000 & 0.000723000 \\ 6 & -3.657216000 & 0.935496000 & -0.001281000 \\ 6 & -3.737729000 & -0.515875000 & -0.001095000 \\ 6 & -4.843259000 & 1.678835000 & -0.001245000 \\ 1 & -4.786834000 & 2.763046000 & -0.001449000 \\ 6 & -2.382899000 & 1.544757000 & -0.001475000 \\ 6 & -2.523781000 & -1.264173000 & -0.001315000 \\ 6 & -6.185973000 & -0.379694000 & -0.000332000 \\ 6 & -6.109930000 & 1.061708000 & -0.000802000 \\ 6 & -4.986598000 & -1.125358000 & -0.000593000 \\ 1 & -5.045600000 & -2.209684000 & -0.000407000 \\ 6 & -7.453531000 & -0.998019000 & 0.000480000 \\ 6 & -7.312260000 & 1.791488000 & -0.000926000 \\ 1 & -7.472277000 & -2.080949000 & 0.000730000 \\ 1 & -2.323118000 & 2.628806000 & -0.001519000 \\ 1 & -2.586237000 & -2.348163000 & -0.001288000 \\ 1 & -7.269379000 & 2.876254000 & -0.001575000 \\ 6 & -1.276828000 & -0.659855000 & -0.001549000 \\ 6 & -1.193095000 & 0.801488000 & -0.001530000 \\ 6 & 1.276840000 & 0.659830000 & -0.001505000 \\ 6 & -0.193110000 & -0.801503000 & -0.001750000 \\ 6 & 0.131843000 & 2.490218000 & -0.001397000 \\ 6 & -2.490238000 & -0.001839000\end{array}$




$\begin{array}{lccc}\mathrm{Q} \mathbf{1}(n=4) & & \\ 6 & -9.800778000 & 1.165099000 & 0.000063000 \\ 6 & 4.959693000 & 0.480109000 & 0.000052000 \\ 6 & 4.890514000 & -0.970531000 & -0.000030000 \\ 6 & -12.358769000 & -0.126020000 & -0.000069000 \\ 6 & -11.183511000 & -0.932248000 & 0.000240000 \\ 6 & -9.864310000 & -0.285516000 & 0.000150000 \\ 6 & 6.209499000 & 1.099838000 & 0.000095000 \\ 1 & 6.259824000 & 2.184586000 & 0.000167000 \\ 6 & 3.744829000 & 1.218932000 & 0.000086000 \\ 6 & -10.996528000 & 1.916862000 & -0.000094000 \\ 1 & -10.930945000 & 3.000099000 & -0.000154000 \\ 6 & -12.265295000 & 1.312006000 & -0.000233000 \\ 6 & 3.621474000 & -1.589552000 & -0.000059000 \\ 6 & -13.651300000 & -0.736652000 & -0.000152000 \\ 6 & -14.812311000 & 0.085878000 & -0.000470000 \\ 1 & -15.787357000 & -0.388736000 & -0.000565000 \\ 6 & -11.337996000 & -2.324352000 & 0.000634000 \\ 6 & -13.746024000 & -2.146295000 & 0.000130000 \\ 1 & -14.726506000 & -2.610011000 & 0.000028000 \\ 6 & -14.704701000 & 1.465795000 & -0.000635000 \\ 1 & -15.600066000 & 2.077460000 & -0.000857000 \\ 6 & -13.451158000 & 2.077682000 & -0.000501000 \\ 1 & -13.374316000 & 3.159585000 & -0.000605000 \\ 6 & -12.602189000 & -2.920824000 & 0.000561000 \\ 1 & -12.679320000 & -4.002390000 & 0.000864000 \\ 6 & 9.864309000 & 0.285518000 & 0.000139000 \\ 6 & 7.342541000 & -1.079547000 & -0.000030000 \\ 6 & 7.407162000 & 0.364438000 & 0.000072000 \\ 6 & 12.358769000 & 0.126025000 & -0.000092000 \\ 6 & 11.183511000 & 0.932252000 & 0.000282000 \\ 6 & 9.800779000 & -1.165099000 & -0.000065000 \\ 6 & 6.084070000 & -1.706001000 & -0.000073000 \\ 1 & 6.035509000 & -2.790573000 & -0.000149000 \\ 6 & 8.552701000 & -1.799752000 & -0.000112000 \\ 6 & 10.996528000 & -1.916859000 & -0.000284000 \\ 1 & 10.930946000 & -3.000097000 & -0.000431000 \\ 6 & 12.265296000 & -1.312002000 & -0.000375000 \\ 6 & 8.675613000 & 0.992556000 & 0.000144000 \\ 6 & 13.651300000 & 0.736656000 & -0.000123000 \\ 6 & 14.812311000 & -0.085873000 & -0.000508000 \\ 1 & 15.787357000 & 0.388741000 & -0.000562000 \\ 6 & 11.337995000 & 2.324355000 & 0.000792000 \\ 6 & 13.746024000 & 2.146300000 & 0.000276000 \\ 1 & 14.726505000 & 2.610015000 & 0.000214000 \\ 6 & 14.704701000 & -1.465790000 & -0.000787000\end{array}$




\begin{tabular}{|c|c|c|c|}
\hline 1 & 15.600066000 & -2.077454000 & -0.001059000 \\
\hline 6 & 13.451158000 & -2.077677000 & -0.000705000 \\
\hline 1 & 13.374316000 & -3.159579000 & -0.000897000 \\
\hline 6 & 12.602189000 & 2.920827000 & 0.000769000 \\
\hline 1 & 12.679319000 & 4.002393000 & 0.001162000 \\
\hline 1 & 8.518356000 & -2.884800000 & -0.000254000 \\
\hline 1 & 3.799068000 & 2.303394000 & 0.000146000 \\
\hline 1 & 10.468378000 & 2.968073000 & 0.001257000 \\
\hline 1 & -10.468379000 & -2.968070000 & 0.001047000 \\
\hline 1 & 3.569446000 & -2.674009000 & -0.000108000 \\
\hline 1 & 8.685386000 & 2.075653000 & 0.000188000 \\
\hline 6 & -6.209498000 & -1.099840000 & 0.000039000 \\
\hline 1 & -6.259822000 & -2.184588000 & 0.000028000 \\
\hline 6 & -7.342542000 & 1.079545000 & 0.000086000 \\
\hline 6 & -7.407165000 & -0.364439000 & 0.000075000 \\
\hline 6 & -6.084069000 & 1.705998000 & 0.000087000 \\
\hline 1 & -6.035506000 & 2.790570000 & 0.000093000 \\
\hline 6 & -8.552700000 & 1.799751000 & 0.000063000 \\
\hline 6 & -8.675613000 & -0.992556000 & 0.000100000 \\
\hline 1 & -8.518354000 & 2.884799000 & 0.000006000 \\
\hline 1 & -8.685387000 & -2.075652000 & 0.000061000 \\
\hline 6 & -0.037352000 & -0.732220000 & 0.000002000 \\
\hline 6 & 0.037356000 & 0.732214000 & 0.000058000 \\
\hline 6 & 2.503904000 & 0.604686000 & 0.000052000 \\
\hline 6 & 2.430750000 & -0.854140000 & -0.000017000 \\
\hline 6 & 1.286882000 & 1.344200000 & 0.000082000 \\
\hline 6 & 1.165597000 & -1.468846000 & -0.000037000 \\
\hline 1 & 1.342457000 & 2.428514000 & 0.000125000 \\
\hline 1 & 1.111668000 & -2.553204000 & -0.000073000 \\
\hline 6 & -1.165599000 & 1.468842000 & 0.000082000 \\
\hline 6 & -1.286883000 & -1.344206000 & -0.000014000 \\
\hline 1 & -1.111670000 & 2.553199000 & 0.000126000 \\
\hline 1 & -1.342460000 & -2.428520000 & -0.000052000 \\
\hline 6 & -4.959696000 & -0.480113000 & 0.000039000 \\
\hline 6 & -4.890516000 & 0.970527000 & 0.000069000 \\
\hline 6 & -2.430748000 & 0.854137000 & 0.000061000 \\
\hline 6 & -2.503902000 & -0.604693000 & 0.000019000 \\
\hline 6 & -3.621473000 & 1.589549000 & 0.000081000 \\
\hline 6 & -3.744828000 & -1.218937000 & 0.000011000 \\
\hline 1 & -3.569445000 & 2.674005000 & 0.000114000 \\
\hline 1 & -3.799067000 & -2.303399000 & -0.000011000 \\
\hline \multicolumn{4}{|c|}{$\mathrm{Q} \mathbf{1}(n=4) \beta \alpha \alpha \alpha$} \\
\hline 6 & 9.801330000 & 1.154756000 & -0.000036000 \\
\hline 6 & -4.956560000 & 0.495130000 & 0.000053000 \\
\hline 6 & -4.882219000 & -0.955116000 & 0.000131000 \\
\hline
\end{tabular}




$\begin{array}{lccc}6 & 12.359755000 & -0.135784000 & -0.000084000 \\ 6 & 11.185834000 & -0.945041000 & -0.000050000 \\ 6 & 9.863360000 & -0.298567000 & -0.000023000 \\ 6 & -6.217531000 & 1.110985000 & 0.000005000 \\ 1 & -6.272245000 & 2.195482000 & -0.000054000 \\ 6 & -3.753846000 & 1.237406000 & 0.000026000 \\ 6 & 10.983659000 & 1.901716000 & -0.000055000 \\ 1 & 10.917760000 & 2.985109000 & -0.000053000 \\ 6 & 12.261890000 & 1.299570000 & -0.000079000 \\ 6 & -3.612887000 & -1.569786000 & 0.000166000 \\ 6 & 13.653647000 & -0.743047000 & -0.000122000 \\ 6 & 14.811855000 & 0.083026000 & -0.000151000 \\ 1 & 15.788538000 & -0.388264000 & -0.000183000 \\ 6 & 11.343548000 & -2.334320000 & -0.000045000 \\ 6 & 13.751640000 & -2.152727000 & -0.000125000 \\ 1 & 14.733145000 & -2.614311000 & -0.000161000 \\ 6 & 14.698814000 & 1.460040000 & -0.000138000 \\ 1 & 15.591044000 & 2.076208000 & -0.000156000 \\ 6 & 13.439755000 & 2.067430000 & -0.000102000 \\ 1 & 13.359605000 & 3.149166000 & -0.000092000 \\ 6 & 12.610228000 & -2.928935000 & -0.000084000 \\ 1 & 12.689133000 & -4.010345000 & -0.000080000 \\ 6 & -9.861317000 & 0.284061000 & -0.000007000 \\ 6 & -7.332052000 & -1.074882000 & 0.000121000 \\ 6 & -7.402904000 & 0.371757000 & 0.000032000 \\ 6 & -12.357549000 & 0.111619000 & -0.000063000 \\ 6 & -11.186766000 & 0.924349000 & -0.000088000 \\ 6 & -9.791404000 & -1.170527000 & 0.000097000 \\ 6 & -6.076072000 & -1.695840000 & 0.000166000 \\ 1 & -6.023542000 & -2.780212000 & 0.000227000 \\ 6 & -8.542734000 & -1.799774000 & 0.000156000 \\ 6 & -10.983494000 & -1.925545000 & 0.000137000 \\ 1 & -10.913840000 & -3.008537000 & 0.000225000 \\ 6 & -12.256537000 & -1.326111000 & 0.000060000 \\ 6 & -8.680817000 & 0.994546000 & -0.000031000 \\ 6 & -13.653200000 & 0.715075000 & -0.000156000 \\ 6 & -14.810079000 & -0.113695000 & -0.000124000 \\ 1 & -15.787608000 & 0.355788000 & -0.000199000 \\ 6 & -11.348843000 & 2.313879000 & -0.000195000 \\ 6 & -13.755850000 & 2.124184000 & -0.000273000 \\ 1 & -14.738914000 & 2.582408000 & -0.000351000 \\ 6 & -14.694860000 & -1.491985000 & 0.000002000 \\ 6 & -15.586562000 & -2.108961000 & 0.000030000 \\ 6 & -2.436796000 & -2.097096000 & 0.000094000 \\ 6 & -3.178611000 & 0.000191000 \\ 6 & 2.904360000 & -0.000288000\end{array}$




$\begin{array}{lccc}1 & -12.699228000 & 3.985522000 & -0.000370000 \\ 1 & -8.504369000 & -2.884650000 & 0.000229000 \\ 1 & -3.811091000 & 2.321682000 & -0.000024000 \\ 1 & -10.482806000 & 2.962520000 & -0.000209000 \\ 1 & 10.475516000 & -2.980417000 & -0.000012000 \\ 1 & -3.557710000 & -2.654138000 & 0.000220000 \\ 1 & -8.694889000 & 2.077630000 & -0.000100000 \\ 6 & 6.214947000 & -1.100506000 & 0.000060000 \\ 1 & 6.261509000 & -2.185425000 & 0.000085000 \\ 6 & 7.345460000 & 1.076531000 & -0.000003000 \\ 6 & 7.404113000 & -0.370934000 & 0.000024000 \\ 6 & 6.072434000 & 1.705969000 & -0.000003000 \\ 1 & 6.028896000 & 2.790866000 & -0.000027000 \\ 6 & 8.540421000 & 1.789799000 & -0.000027000 \\ 6 & 8.678152000 & -1.001750000 & 0.000010000 \\ 1 & 8.508252000 & 2.875065000 & -0.000042000 \\ 1 & 8.684714000 & -2.085025000 & 0.000027000 \\ 6 & 0.028230000 & -0.708927000 & 0.000118000 \\ 6 & -0.044587000 & 0.754268000 & 0.000060000 \\ 6 & -2.505778000 & 0.624497000 & 0.000062000 \\ 6 & -2.430012000 & -0.830630000 & 0.000130000 \\ 6 & -1.294027000 & 1.366959000 & 0.000034000 \\ 6 & -1.153170000 & -1.443553000 & 0.000155000 \\ 6 & -1.350365000 & 2.451215000 & -0.000009000 \\ 6 & -4.938531000 & 0.484604000 & 0.000053000 \\ 6 & -4.868508000 & -0.975315000 & 0.000129000 \\ 6 & 1.098686000 & -2.528044000 & 0.000202000 \\ 6 & 1.159449000 & 1.490940000 & 0.000032000 \\ 1 & 1.301667000 & -1.321987000 & 0.000132000 \\ 1 & 1.108133000 & 2.575364000 & -0.000003000 \\ 6 & 1.354578000 & -2.406591000 & 0.000172000 \\ 6 & 4.952052000 & -0.477815000 & 0.000063000 \\ 6 & 2.891647000 & 0.979699000 & 0.000026000 \\ 6 & 2.491712000 & -0.591042000 & 0.000095000 \\ 6 & 3.612318000 & 1.602918000 & 0.000019000 \\ 6 & 3.566090000 & 2.687623000 & -0.000011000 \\ 6 & -0.125654000 & -0.000059000 \\ 6 & -0.937460000 & -0.000199000 \\ 6 & -0.293799000 & -0.000098000 \\ 6 & 1.102208000 & -0.000003000\end{array}$




\begin{tabular}{cccc}
1 & -6.272755000 & 2.186597000 & -0.000065000 \\
6 & -3.761648000 & 1.219018000 & 0.000033000 \\
6 & 10.974111000 & 1.912144000 & 0.000040000 \\
1 & 10.908251000 & 2.995495000 & 0.000096000 \\
6 & 12.255348000 & 1.309505000 & 0.000070000 \\
6 & -3.618286000 & -1.592627000 & 0.000168000 \\
6 & 13.649464000 & -0.730519000 & -0.000083000 \\
6 & 14.806485000 & 0.097226000 & 0.000068000 \\
1 & 15.783945000 & -0.372432000 & 0.000064000 \\
6 & 11.342053000 & -2.324595000 & -0.000440000 \\
6 & 13.749945000 & -2.140447000 & -0.000276000 \\
1 & 14.732195000 & -2.600352000 & -0.000286000 \\
6 & 14.691247000 & 1.472726000 & 0.000209000 \\
1 & 15.582296000 & 2.090632000 & 0.000318000 \\
6 & 13.429520000 & 2.077806000 & 0.000201000 \\
1 & 13.347184000 & 3.159441000 & 0.000291000 \\
6 & 12.610397000 & -2.917744000 & -0.000469000 \\
1 & 12.690594000 & -3.999070000 & -0.000650000 \\
6 & -9.855202000 & 0.293799000 & -0.000087000 \\
6 & -7.327012000 & -1.085613000 & 0.000104000 \\
6 & -7.391747000 & 0.370408000 & 0.000009000 \\
6 & -12.354713000 & 0.125652000 & -0.000036000 \\
6 & -11.181319000 & 0.937465000 & -0.000242000 \\
6 & -9.791730000 & -1.167299000 & 0.000088000 \\
6 & -6.075880000 & -1.711099000 & 0.000158000 \\
1 & -6.029672000 & -2.795546000 & 0.000223000 \\
6 & -8.536639000 & -1.802091000 & 0.000154000 \\
6 & -10.974110000 & -1.912145000 & 0.000229000 \\
1 & -10.908249000 & -2.995496000 & 0.000372000 \\
6 & -12.255347000 & -1.309506000 & 0.000212000 \\
6 & -8.676969000 & 0.996533000 & -0.000079000 \\
6 & -13.649465000 & 0.730517000 & -0.000112000 \\
6 & -14.806485000 & -0.097228000 & 0.000107000 \\
1 & -15.783945000 & 0.372430000 & 0.000063000 \\
6 & -11.342055000 & 2.324594000 & -0.000599000 \\
6 & -13.749947000 & 2.140445000 & -0.000423000 \\
1 & -14.732197000 & 2.600350000 & -0.000472000 \\
6 & -14.691247000 & -1.472728000 & 0.000362000 \\
1 & -15.582295000 & -2.090634000 & 0.000522000 \\
6 & -13.429520000 & -2.077807000 & 0.000405000 \\
1 & -13.347183000 & -3.159443000 & 0.000584000 \\
6 & -12.610399000 & 2.917742000 & -0.000679000 \\
1 & -12.690596000 & 3.999069000 & -0.000950000 \\
1 & -8.504015000 & -2.887051000 & 0.000264000 \\
\hline & -10.818906000 & 2.303100000 & -0.000019000 \\
6
\end{tabular}




$\begin{array}{lrrr}1 & 10.475259000 & -2.972564000 & -0.000626000 \\ 1 & -3.567783000 & -2.676946000 & 0.000219000 \\ 1 & -8.686989000 & 2.079730000 & -0.000137000 \\ 6 & 6.219302000 & -1.102207000 & 0.000053000 \\ 1 & 6.272754000 & -2.186596000 & 0.000073000 \\ 6 & 7.327013000 & 1.085614000 & -0.000012000 \\ 6 & 7.391747000 & -0.370407000 & 0.000006000 \\ 6 & 6.075881000 & 1.711100000 & -0.000002000 \\ 1 & 6.029673000 & 2.795547000 & -0.000018000 \\ 6 & 8.536640000 & 1.802091000 & -0.000021000 \\ 6 & 8.676969000 & -0.996533000 & -0.000034000 \\ 1 & 8.504017000 & 2.887051000 & 0.000005000 \\ 1 & 8.686988000 & -2.079730000 & -0.000010000 \\ 6 & 0.037285000 & -0.727731000 & 0.000129000 \\ 6 & -0.037286000 & 0.727733000 & 0.000074000 \\ 6 & -2.486537000 & 0.604334000 & 0.000073000 \\ 6 & -2.413019000 & -0.853081000 & 0.000137000 \\ 6 & -1.302526000 & 1.341775000 & 0.000048000 \\ 6 & -1.158639000 & -1.467620000 & 0.000163000 \\ 1 & -1.359385000 & 2.425921000 & 0.000004000 \\ 1 & -1.104579000 & -2.551889000 & 0.000206000 \\ 6 & 1.158640000 & 1.467622000 & 0.000045000 \\ 6 & 1.302526000 & -1.341773000 & 0.000143000 \\ 1 & 1.104579000 & 2.551891000 & 0.000008000 \\ 1 & 1.359384000 & -2.425919000 & 0.000180000 \\ 6 & 4.938531000 & -0.484603000 & 0.000066000 \\ 6 & 4.868508000 & 0.975317000 & 0.000031000 \\ 6 & 2.413019000 & 0.853083000 & 0.000060000 \\ 6 & 2.486537000 & -0.604333000 & 0.000106000 \\ 6 & 3.618287000 & 1.592629000 & 0.000029000 \\ 6 & 3.761648000 & -1.219016000 & 0.000107000 \\ 1 & 3.567783000 & 2.676947000 & -0.000001000 \\ 1 & 3.818906000 & -2.303098000 & 0.000136000 \\ & & & \\ \mathrm{Q} \mathbf{1}(n=4) 83 \alpha 0 & & \\ 6 & 9.796629000 & 1.163160000 & -0.000032000 \\ 6 & -4.948963000 & 0.489062000 & 0.000075000 \\ 6 & -4.879922000 & -0.966901000 & 0.000150000 \\ 6 & 12.359370000 & -0.122746000 & -0.000092000 \\ 6 & 11.186695000 & -0.935283000 & -0.000159000 \\ 6 & 9.862443000 & -0.292912000 & -0.000072000 \\ 6 & -6.219681000 & 1.107505000 & 0.000017000 \\ 1 & -6.271921000 & 2.192052000 & -0.000043000 \\ 6 & -3.760060000 & 1.224125000 & 0.000056000 \\ 6 & 10.977692000 & 1.912272000 & 0.000008000 \\ 1 & 10.909370000 & 2.995494000 & 0.000052000\end{array}$




$\begin{array}{lccc}6 & 12.258674000 & 1.312064000 & 0.000004000 \\ 6 & -3.614036000 & -1.585388000 & 0.000191000 \\ 6 & 13.654642000 & -0.726917000 & -0.000141000 \\ 6 & 14.810872000 & 0.101801000 & -0.000062000 \\ 1 & 15.788704000 & -0.367096000 & -0.000092000 \\ 6 & 11.348073000 & -2.323686000 & -0.000317000 \\ 6 & 13.755856000 & -2.136685000 & -0.000275000 \\ 1 & 14.738402000 & -2.595972000 & -0.000312000 \\ 6 & 14.694706000 & 1.478018000 & 0.000047000 \\ 1 & 15.585489000 & 2.096309000 & 0.000106000 \\ 6 & 13.433450000 & 2.082257000 & 0.000074000 \\ 1 & 13.350253000 & 3.163809000 & 0.000144000 \\ 6 & 12.616393000 & -2.915303000 & -0.000370000 \\ 1 & 12.697740000 & -3.996550000 & -0.000488000 \\ 6 & -9.862443000 & 0.292912000 & -0.000061000 \\ 6 & -7.335784000 & -1.076929000 & 0.000122000 \\ 6 & -7.401416000 & 0.373695000 & 0.000028000 \\ 6 & -12.359370000 & 0.122745000 & -0.000069000 \\ 6 & -11.186696000 & 0.935282000 & -0.000202000 \\ 6 & -9.796628000 & -1.163160000 & 0.000095000 \\ 6 & -6.073418000 & -1.701690000 & 0.000177000 \\ 1 & -6.025408000 & -2.786237000 & 0.000239000 \\ 6 & -8.537388000 & -1.795560000 & 0.000164000 \\ 6 & -10.977692000 & -1.912273000 & 0.000198000 \\ 1 & -10.909369000 & -2.995495000 & 0.000327000 \\ 6 & -12.258674000 & -1.312066000 & 0.000146000 \\ 6 & -8.680635000 & 0.999227000 & -0.000058000 \\ 6 & -13.654643000 & 0.726916000 & -0.000170000 \\ 6 & -14.810872000 & -0.101803000 & -0.000023000 \\ 1 & -15.788704000 & 0.367094000 & -0.000093000 \\ 6 & -11.348074000 & 2.323685000 & -0.000477000 \\ 6 & -13.755857000 & 2.136683000 & -0.000421000 \\ 1 & -14.738403000 & 2.595970000 & -0.000498000 \\ 6 & -14.694706000 & -1.478020000 & 0.000200000 \\ 1 & -15.585489000 & -2.096311000 & 0.000311000 \\ 6 & -13.433450000 & -2.082259000 & 0.000279000 \\ 1 & -13.350252000 & -3.163811000 & 0.000438000 \\ 6 & -12.616394000 & 2.915302000 & -0.000580000 \\ 1 & -12.697741000 & 3.996548000 & -0.000788000 \\ 1 & -8.502191000 & -2.880581000 & 0.000258000 \\ 6 & -3.815165000 & 2.308435000 & 0.000007000 \\ 6 & -10.481540000 & 2.971938000 & -0.000627000 \\ 6 & -2.961539000 & -2.971939000 & -0.000413000 \\ 6 & -2.669863000 & 0.000243000 \\ 6 & -1.107504000 & 0.000073000\end{array}$




$\begin{array}{lrrc}1 & 6.271921000 & -2.192051000 & 0.000095000 \\ 6 & 7.335785000 & 1.076930000 & 0.000007000 \\ 6 & 7.401415000 & -0.373694000 & 0.000025000 \\ 6 & 6.073419000 & 1.701691000 & 0.000018000 \\ 1 & 6.025409000 & 2.786238000 & -0.000002000 \\ 6 & 8.537388000 & 1.795560000 & -0.000011000 \\ 6 & 8.680635000 & -0.999227000 & -0.000014000 \\ 1 & 8.502192000 & 2.880581000 & -0.000001000 \\ 1 & 8.691879000 & -2.082458000 & 0.000007000 \\ 6 & 0.036269000 & -0.728790000 & 0.000155000 \\ 6 & -0.036269000 & 0.728792000 & 0.000100000 \\ 6 & -2.493371000 & 0.608165000 & 0.000097000 \\ 6 & -2.421769000 & -0.849058000 & 0.000162000 \\ 6 & -1.302197000 & 1.344293000 & 0.000073000 \\ 6 & -1.155767000 & -1.465462000 & 0.000188000 \\ 1 & -1.357155000 & 2.428651000 & 0.000030000 \\ 1 & -1.102879000 & -2.549913000 & 0.000234000 \\ 6 & 1.155767000 & 1.465463000 & 0.000071000 \\ 6 & 1.302197000 & -1.344292000 & 0.000168000 \\ 1 & 1.102879000 & 2.549915000 & 0.000037000 \\ 1 & 1.357154000 & -2.428649000 & 0.000205000 \\ 6 & 4.948963000 & -0.489061000 & 0.000088000 \\ 6 & 4.879922000 & 0.966902000 & 0.000052000 \\ 6 & 2.421769000 & 0.849060000 & 0.000084000 \\ 6 & 2.493371000 & -0.608163000 & 0.000130000 \\ 6 & 3.614036000 & 1.585389000 & 0.000052000 \\ 6 & 3.760060000 & -1.224123000 & 0.000130000 \\ 1 & 3.563266000 & 2.669865000 & 0.000023000 \\ 1 & 3.815165000 & -2.308434000 & 0.000161000 \\ & & & \\ \mathrm{Q} \mathbf{1}(n=4) \alpha \beta \beta \alpha & & \\ 6 & 9.792268000 & 1.158276000 & -0.000034000 \\ 6 & -4.954123000 & 0.499303000 & 0.000058000 \\ 6 & -4.886083000 & -0.954996000 & 0.000135000 \\ 6 & 12.360141000 & -0.118909000 & -0.000073000 \\ 6 & 11.191166000 & -0.935190000 & -0.000161000 \\ 6 & 9.863604000 & -0.296904000 & -0.000076000 \\ 6 & -6.222567000 & 1.117735000 & 0.000002000 \\ 1 & -6.274756000 & 2.202303000 & -0.000060000 \\ 6 & -3.762006000 & 1.233392000 & 0.000037000 \\ 6 & 10.972585000 & 1.911659000 & 0.000019000 \\ 1 & 10.900087000 & 2.994585000 & 0.000065000 \\ 6 & 12.253709000 & 1.316325000 & 0.000028000 \\ 6 & -3.603302000 & -1.573360000 & 0.000174000 \\ 6 & 13.657505000 & -0.718188000 & -0.000110000 \\ 6 & 14.811050000 & 0.114811000 & -0.000011000\end{array}$




\begin{tabular}{|c|c|c|c|}
\hline & 15.790504000 & -0.350636000 & -0.000030000 \\
\hline 6 & 11.356960000 & -2.322710000 & -0.000336000 \\
\hline 6 & 13.763922000 & -2.127268000 & -0.000256000 \\
\hline 1 & 14.748128000 & -2.582987000 & -0.000283000 \\
\hline 6 & 14.689869000 & 1.490841000 & 0.000102000 \\
\hline 1 & 15.578522000 & 2.112189000 & 0.000176000 \\
\hline 6 & 13.426967000 & 2.090675000 & 0.000116000 \\
\hline 1 & 13.340012000 & 3.171910000 & 0.000189000 \\
\hline 6 & 12.627263000 & -2.910071000 & -0.000378000 \\
\hline 1 & 12.712331000 & -3.991018000 & -0.000509000 \\
\hline 6 & -9.863601000 & 0.296899000 & -0.000066000 \\
\hline 6 & -7.335791000 & -1.065406000 & 0.000112000 \\
\hline 6 & -7.404233000 & 0.382378000 & 0.000016000 \\
\hline 6 & -12.360141000 & 0.118890000 & -0.000050000 \\
\hline 6 & -11.191171000 & 0.935177000 & -0.000204000 \\
\hline 6 & -9.792257000 & -1.158287000 & 0.000093000 \\
\hline 6 & -6.065504000 & -1.687411000 & 0.000165000 \\
\hline 1 & -6.015749000 & -2.772028000 & 0.000229000 \\
\hline 6 & -8.532057000 & -1.786160000 & 0.000159000 \\
\hline 6 & -10.972580000 & -1.911675000 & 0.000209000 \\
\hline 1 & -10.900081000 & -2.994600000 & 0.000341000 \\
\hline 6 & -12.253705000 & -1.316345000 & 0.000170000 \\
\hline 6 & -8.685067000 & 1.006206000 & -0.000067000 \\
\hline 6 & -13.657507000 & 0.718162000 & -0.000139000 \\
\hline 6 & -14.811050000 & -0.114843000 & 0.000028000 \\
\hline 1 & -15.790506000 & 0.350601000 & -0.000031000 \\
\hline 6 & -11.356972000 & 2.322694000 & -0.000496000 \\
\hline 6 & -13.763932000 & 2.127241000 & -0.000403000 \\
\hline 1 & -14.748142000 & 2.582955000 & -0.000469000 \\
\hline 6 & -14.689865000 & -1.490871000 & 0.000257000 \\
\hline 1 & -15.578514000 & -2.112225000 & 0.000381000 \\
\hline 6 & -13.426958000 & -2.090701000 & 0.000321000 \\
\hline 1 & -13.340001000 & -3.171935000 & 0.000483000 \\
\hline 6 & -12.627279000 & 2.910051000 & -0.000588000 \\
\hline 1 & -12.712351000 & 3.990997000 & -0.000810000 \\
\hline 1 & -8.493312000 & -2.871130000 & 0.000259000 \\
\hline 1 & -3.815832000 & 2.317805000 & -0.000014000 \\
\hline 1 & -10.492574000 & 2.973871000 & -0.000668000 \\
\hline 1 & 10.492557000 & -2.973881000 & -0.000454000 \\
\hline 1 & -3.553077000 & -2.657977000 & 0.000227000 \\
\hline 1 & -8.698186000 & 2.089440000 & -0.000131000 \\
\hline 6 & 6.222560000 & -1.117725000 & 0.000058000 \\
\hline 1 & 6.274751000 & -2.202294000 & 0.000078000 \\
\hline 6 & 7.335808000 & 1.065409000 & -0.000002000 \\
\hline 6 & 7.404238000 & -0.382373000 & 0.000014000 \\
\hline 6 & 6.065498000 & 1.687421000 & 0.000007000 \\
\hline
\end{tabular}




$\begin{array}{rrrr}1 & 6.015759000 & 2.772039000 & -0.000011000 \\ 6 & 8.532055000 & 1.786156000 & -0.000015000 \\ 6 & 8.685059000 & -1.006209000 & -0.000022000 \\ 1 & 8.493319000 & 2.871128000 & 0.000001000 \\ 1 & 8.698177000 & -2.089443000 & -0.000003000 \\ 6 & 0.034384000 & -0.732526000 & 0.000134000 \\ 6 & -0.034402000 & 0.732561000 & 0.000079000 \\ 6 & -2.497659000 & 0.617111000 & 0.000077000 \\ 6 & -2.429369000 & -0.843875000 & 0.000143000 \\ 6 & -1.306923000 & 1.348381000 & 0.000053000 \\ 6 & -1.146082000 & -1.461219000 & 0.000168000 \\ 1 & -1.360207000 & 2.432832000 & 0.000010000 \\ 1 & -1.094944000 & -2.545772000 & 0.000214000 \\ 6 & 1.146094000 & 1.461254000 & 0.000052000 \\ 6 & 1.306939000 & -1.348346000 & 0.000147000 \\ 1 & 1.094947000 & 2.545807000 & 0.000017000 \\ 1 & 1.360210000 & -2.432797000 & 0.000185000 \\ 6 & 4.954124000 & -0.499291000 & 0.000071000 \\ 6 & 4.886095000 & 0.955018000 & 0.000038000 \\ 6 & 2.429363000 & 0.843911000 & 0.000066000 \\ 6 & 2.497649000 & -0.617088000 & 0.000110000 \\ 6 & 3.603301000 & 1.573385000 & 0.000037000 \\ 6 & 3.762010000 & -1.233368000 & 0.000111000 \\ 1 & 3.553083000 & 2.658001000 & 0.000009000 \\ 1 & 3.815832000 & -2.317781000 & 0.000140000\end{array}$

$\begin{array}{lccc}\mathrm{Q} \mathbf{1} & (n=5) & & \\ 6 & -11.034880000 & 1.160503000 & -0.000052000 \\ 6 & 6.181898000 & 0.454106000 & -0.001557000 \\ 6 & 6.122205000 & -0.997019000 & -0.001948000 \\ 6 & -13.585734000 & -0.148459000 & 0.002006000 \\ 6 & -12.405713000 & -0.948142000 & 0.003390000 \\ 6 & -11.088714000 & -0.293301000 & 0.001438000 \\ 6 & 7.433446000 & 1.082153000 & -0.000795000 \\ 1 & 7.476545000 & 2.167207000 & -0.000393000 \\ 6 & 4.968331000 & 1.184627000 & -0.001853000 \\ 6 & -12.233576000 & 1.902830000 & -0.000859000 \\ 1 & -12.175644000 & 2.986531000 & -0.002109000 \\ 6 & -13.500708000 & 1.289772000 & -0.000289000 \\ 6 & 4.859491000 & -1.624006000 & -0.002427000 \\ 6 & -14.874586000 & -0.766436000 & 0.003255000 \\ 6 & -16.040374000 & 0.049471000 & 0.001708000 \\ 1 & -17.012676000 & -0.430766000 & 0.002551000 \\ 6 & -12.552245000 & -2.339910000 & 0.006798000 \\ 6 & -14.961303000 & -2.176711000 & 0.006173000\end{array}$




\begin{tabular}{|c|c|c|c|}
\hline & -15.939137000 & -2.646012000 & 0.007070000 \\
\hline 6 & -15.940434000 & 1.429029000 & -0.000716000 \\
\hline 1 & -16.838887000 & 2.036124000 & -0.001841000 \\
\hline 6 & -14.689138000 & 2.047978000 & -0.001613000 \\
\hline 1 & -14.618636000 & 3.130332000 & -0.003295000 \\
\hline 6 & -13.813424000 & -2.944225000 & 0.008084000 \\
\hline 1 & -13.883932000 & -4.026232000 & 0.010680000 \\
\hline 6 & 11.088714000 & 0.293301000 & 0.001446000 \\
\hline 6 & 8.574782000 & -1.091289000 & -0.001004000 \\
\hline 6 & 8.629837000 & 0.355292000 & -0.000356000 \\
\hline 6 & 13.585734000 & 0.148460000 & 0.002016000 \\
\hline 6 & 12.405713000 & 0.948143000 & 0.003400000 \\
\hline 6 & 11.034880000 & -1.160503000 & -0.000043000 \\
\hline 6 & 7.322945000 & -1.725509000 & -0.001737000 \\
\hline 1 & 7.281113000 & -2.810351000 & -0.002117000 \\
\hline 6 & 9.790712000 & -1.803110000 & -0.000998000 \\
\hline 6 & 12.233576000 & -1.902830000 & -0.000849000 \\
\hline 1 & 12.175644000 & -2.986530000 & -0.002099000 \\
\hline 6 & 13.500708000 & -1.289772000 & -0.000278000 \\
\hline 6 & 9.898528000 & 0.991448000 & 0.000821000 \\
\hline 6 & 14.874586000 & 0.766437000 & 0.003266000 \\
\hline 6 & 16.040374000 & -0.049470000 & 0.001720000 \\
\hline 1 & 17.012676000 & 0.430767000 & 0.002564000 \\
\hline 6 & 12.552245000 & 2.339910000 & 0.006807000 \\
\hline 6 & 14.961303000 & 2.176711000 & 0.006184000 \\
\hline 1 & 15.939137000 & 2.646013000 & 0.007081000 \\
\hline 6 & 15.940434000 & -1.429028000 & -0.000703000 \\
\hline 1 & 16.838887000 & -2.036124000 & -0.001827000 \\
\hline 6 & 14.689137000 & -2.047977000 & -0.001600000 \\
\hline 1 & 14.618637000 & -3.130332000 & -0.003283000 \\
\hline 6 & 13.813424000 & 2.944226000 & 0.008094000 \\
\hline 1 & 13.883932000 & 4.026233000 & 0.010689000 \\
\hline 1 & 9.763696000 & -2.888360000 & -0.001897000 \\
\hline 1 & 5.015383000 & 2.269443000 & -0.001595000 \\
\hline 1 & 11.678999000 & 2.978788000 & 0.008743000 \\
\hline 1 & -11.678999000 & -2.978787000 & 0.008735000 \\
\hline 1 & 4.813730000 & -2.708748000 & -0.002611000 \\
\hline 1 & 9.900918000 & 2.074632000 & 0.001186000 \\
\hline 6 & -6.181898000 & -0.454106000 & -0.001562000 \\
\hline 6 & -6.122205000 & 0.997018000 & -0.001954000 \\
\hline 6 & -7.433446000 & -1.082153000 & -0.000800000 \\
\hline 1 & -7.476546000 & -2.167208000 & -0.000398000 \\
\hline 6 & -4.968331000 & -1.184628000 & -0.001856000 \\
\hline 6 & -4.859491000 & 1.624006000 & -0.002431000 \\
\hline 6 & -8.574782000 & 1.091289000 & -0.001012000 \\
\hline 6 & -8.629837000 & -0.355292000 & -0.000362000 \\
\hline
\end{tabular}




$\begin{array}{lccc}6 & -7.322945000 & 1.725508000 & -0.001743000 \\ 1 & -7.281113000 & 2.810350000 & -0.002124000 \\ 6 & -9.790712000 & 1.803110000 & -0.001006000 \\ 6 & -9.898528000 & -0.991448000 & 0.000813000 \\ 1 & -9.763696000 & 2.888360000 & -0.001906000 \\ 1 & -5.015383000 & -2.269444000 & -0.001598000 \\ 1 & -4.813729000 & 2.708747000 & -0.002616000 \\ 1 & -9.900918000 & -2.074632000 & 0.001179000 \\ 6 & 1.200705000 & -0.788123000 & -0.002810000 \\ 6 & 1.267540000 & 0.675692000 & -0.002743000 \\ 6 & 3.729226000 & 0.561818000 & -0.002333000 \\ 6 & 3.665137000 & -0.894715000 & -0.002557000 \\ 6 & 2.507381000 & 1.294739000 & -0.002517000 \\ 6 & 2.400796000 & -1.517706000 & -0.002784000 \\ 1 & 2.556790000 & 2.379404000 & -0.002387000 \\ 1 & 2.353238000 & -2.602379000 & -0.002831000 \\ 6 & 0.052705000 & 1.407418000 & -0.002820000 \\ 6 & -0.052705000 & -1.407419000 & -0.002820000 \\ 1 & 0.102033000 & 2.492023000 & -0.002783000 \\ 1 & -0.102033000 & -2.492023000 & -0.002782000 \\ 6 & -3.729226000 & -0.561819000 & -0.002336000 \\ 6 & -3.665137000 & 0.894714000 & -0.002560000 \\ 6 & -1.200704000 & 0.788122000 & -0.002812000 \\ 6 & -1.267540000 & -0.675693000 & -0.002743000 \\ 6 & -2.400796000 & 1.517705000 & -0.002787000 \\ 6 & -2.507382000 & -1.294740000 & -0.002519000 \\ 6 & -2.353238000 & 2.602378000 & -0.002834000 \\ 6 & -14.875774000 & -0.773598000 & 0.000084000 \\ 6 & -17.556790000 & -2.379405000 & -0.002388000 \\ 6 & -12.224940000 & 1.889246000 & -0.000113000 \\ 6 & -13.495799000 & 2.972956000 & -0.000195000 \\ 6 & -11.032719000 & 1.148900000 & -0.000030000 \\ 6 & 6.179851000 & 0.468813000 & -0.000034000 \\ 6 & -13.585884000 & -0.158631000 & 0.000032000 \\ 6 & -12.407446000 & -0.960685000 & 0.000130000 \\ 6 & -11.087405000 & -0.306790000 & 0.000044000 \\ 6 & 7.488452000 & 1.092146000 & -0.000009000 \\ 6 & -0.432458000 & 0.000005000\end{array}$




$\begin{array}{cccc}6 & -12.556306000 & -2.350305000 & 0.000317000 \\ 6 & -14.965256000 & -2.183746000 & 0.000255000 \\ 1 & -15.943958000 & -2.651220000 & 0.000296000 \\ 6 & -15.935695000 & 1.423024000 & -0.000168000 \\ 1 & -16.832000000 & 2.033271000 & -0.000245000 \\ 6 & -14.680811000 & 2.038555000 & -0.000205000 \\ 1 & -14.607544000 & 3.120776000 & -0.000304000 \\ 6 & -13.819285000 & -2.952981000 & 0.000375000 \\ 1 & -13.891315000 & -4.034871000 & 0.000521000 \\ 6 & 11.087180000 & 0.289016000 & 0.000049000 \\ 6 & 8.565877000 & -1.087726000 & -0.000038000 \\ 6 & 8.627462000 & 0.360814000 & -0.000005000 \\ 6 & 13.585020000 & 0.131686000 & 0.000049000 \\ 6 & 12.409089000 & 0.937541000 & 0.000121000 \\ 6 & 11.026822000 & -1.167170000 & -0.000020000 \\ 6 & 7.314610000 & -1.716397000 & -0.000061000 \\ 1 & 7.268914000 & -2.801034000 & -0.000082000 \\ 6 & 9.780779000 & -1.804438000 & -0.000052000 \\ 6 & 12.221561000 & -1.913552000 & -0.000069000 \\ 1 & 12.159117000 & -2.997000000 & -0.000127000 \\ 6 & 13.492702000 & -1.306120000 & -0.000053000 \\ 6 & 9.903112000 & 0.991421000 & 0.000037000 \\ 6 & 14.876986000 & 0.742879000 & 0.000093000 \\ 6 & 16.038690000 & -0.079026000 & 0.000014000 \\ 1 & 17.013450000 & 0.396194000 & 0.000043000 \\ 6 & 12.562937000 & 2.327509000 & 0.000266000 \\ 6 & 14.971141000 & 2.152701000 & 0.000220000 \\ 6 & -6.177954000 & -0.455877000 & -0.000042000 \\ 6 & -6.125337000 & 0.997917000 & -0.000039000 \\ 6 & -7.436282000 & -1.087729000 & -0.000029000 \\ 1 & 15.951410000 & 2.616877000 & 0.000251000 \\ 1 & 15.931481000 & -1.457395000 & -0.000093000 \\ 6 & 16.826569000 & -2.069434000 & -0.000151000 \\ 1 & 14.676181000 & -2.069802000 & -0.000124000 \\ 1 & 14.600156000 & -3.151799000 & -0.000198000 \\ 1 & 13.827547000 & 2.925786000 & 0.000311000 \\ 6 & 9.7496730000 & -2.889526000 & -0.000095000 \\ 6 & 11.693069000 & 2.971049000 & 0.000353000 \\ 6 & -2.172866000 & -0.000026000 \\ 6 & -1.179601000 & -0.000054000 \\ 6 & 1.629384000 & -0.000041000\end{array}$




\begin{tabular}{|c|c|c|c|}
\hline 6 & -8.575486000 & 1.082826000 & -0.000027000 \\
\hline 6 & -8.627833000 & -0.364598000 & -0.000012000 \\
\hline 6 & -7.314448000 & 1.718501000 & -0.000034000 \\
\hline 1 & -7.275515000 & 2.803563000 & -0.000035000 \\
\hline 6 & -9.782676000 & 1.790687000 & -0.000044000 \\
\hline 6 & -9.900664000 & -1.002908000 & 0.000022000 \\
\hline 1 & -9.755972000 & 2.876032000 & -0.000069000 \\
\hline 1 & -5.016867000 & -2.264524000 & -0.000057000 \\
\hline 1 & -4.810439000 & 2.714339000 & -0.000035000 \\
\hline 1 & -9.901169000 & -2.086211000 & 0.000035000 \\
\hline 6 & 1.204303000 & -0.761048000 & -0.000066000 \\
\hline 6 & 1.272183000 & 0.700658000 & -0.000053000 \\
\hline 6 & 3.729888000 & 0.583333000 & -0.000048000 \\
\hline 6 & 3.661322000 & -0.871163000 & -0.000065000 \\
\hline 6 & 2.514619000 & 1.319340000 & -0.000045000 \\
\hline 6 & 2.390626000 & -1.490963000 & -0.000072000 \\
\hline 1 & 2.565965000 & 2.403869000 & -0.000034000 \\
\hline 1 & 2.340305000 & -2.575592000 & -0.000080000 \\
\hline 6 & 0.058431000 & 1.433230000 & -0.000047000 \\
\hline 6 & -0.065144000 & -1.380334000 & -0.000070000 \\
\hline 1 & 0.106383000 & 2.517803000 & -0.000038000 \\
\hline 1 & -0.114811000 & -2.465032000 & -0.000074000 \\
\hline 6 & -3.721689000 & -0.552657000 & -0.000057000 \\
\hline 6 & -3.665990000 & 0.908975000 & -0.000047000 \\
\hline 6 & -1.196337000 & 0.811711000 & -0.000051000 \\
\hline 6 & -1.258705000 & -0.654779000 & -0.000063000 \\
\hline 6 & -2.393917000 & 1.536822000 & -0.000045000 \\
\hline 6 & -2.517485000 & -1.276671000 & -0.000065000 \\
\hline 1 & -2.351008000 & 2.621564000 & -0.000036000 \\
\hline 1 & -2.564482000 & -2.361456000 & -0.000069000 \\
\hline \multicolumn{4}{|c|}{$\mathrm{Q} \mathbf{1}(n=5) \alpha \beta \alpha \alpha$} \\
\hline 6 & -11.024584000 & 1.166010000 & 0.000001000 \\
\hline 6 & 6.174807000 & 0.460487000 & 0.000027000 \\
\hline 6 & 6.117773000 & -0.996497000 & 0.000042000 \\
\hline 6 & -13.584434000 & -0.140353000 & -0.000041000 \\
\hline 6 & -12.408319000 & -0.944963000 & -0.000065000 \\
\hline 6 & -11.083856000 & -0.295300000 & -0.000030000 \\
\hline 6 & 7.439456000 & 1.088374000 & 0.000010000 \\
\hline 1 & 7.482666000 & 2.173376000 & -0.000002000 \\
\hline 6 & 4.978787000 & 1.187122000 & 0.000027000 \\
\hline 6 & -12.225334000 & 1.908901000 & 0.000018000 \\
\hline 1 & -12.165783000 & 2.992522000 & 0.000044000 \\
\hline 6 & -13.493954000 & 1.298820000 & 0.000004000 \\
\hline 6 & 4.853717000 & -1.624507000 & 0.000051000 \\
\hline 6 & -14.874942000 & -0.752819000 & -0.000066000 \\
\hline
\end{tabular}




\begin{tabular}{|c|c|c|c|}
\hline 6 & -16.038637000 & 0.067459000 & -0.000039000 \\
\hline 1 & -17.012328000 & -0.409944000 & -0.000057000 \\
\hline 6 & -12.559767000 & -2.333435000 & -0.000125000 \\
\hline 6 & -14.967966000 & -2.162872000 & -0.000120000 \\
\hline 1 & -15.948029000 & -2.627508000 & -0.000139000 \\
\hline 6 & -15.933566000 & 1.445383000 & 0.000008000 \\
\hline 1 & -16.829109000 & 2.056715000 & 0.000027000 \\
\hline 6 & -14.678565000 & 2.059905000 & 0.000028000 \\
\hline 1 & -14.605095000 & 3.142068000 & 0.000061000 \\
\hline 6 & -13.824184000 & -2.934376000 & -0.000151000 \\
\hline 1 & -13.897653000 & -4.016144000 & -0.000196000 \\
\hline 6 & 11.089639000 & 0.301362000 & -0.000017000 \\
\hline 6 & 8.574675000 & -1.087308000 & 0.000030000 \\
\hline 6 & 8.628205000 & 0.363416000 & 0.000009000 \\
\hline 6 & 13.587723000 & 0.150459000 & -0.000032000 \\
\hline 6 & 12.408759000 & 0.954251000 & -0.000052000 \\
\hline 6 & 11.035703000 & -1.154565000 & 0.000016000 \\
\hline 6 & 7.313767000 & -1.721773000 & 0.000045000 \\
\hline 1 & 7.274840000 & -2.806736000 & 0.000058000 \\
\hline 6 & 9.779003000 & -1.796544000 & 0.000036000 \\
\hline 6 & 12.220892000 & -1.894859000 & 0.000032000 \\
\hline 1 & 12.162543000 & -2.978648000 & 0.000060000 \\
\hline 6 & 13.498474000 & -1.284701000 & 0.000014000 \\
\hline 6 & 9.901721000 & 0.998671000 & -0.000014000 \\
\hline 6 & 14.878282000 & 0.764661000 & -0.000060000 \\
\hline 6 & 16.040818000 & -0.055304000 & -0.000037000 \\
\hline 1 & 17.014905000 & 0.421436000 & -0.000058000 \\
\hline 6 & 12.560075000 & 2.343840000 & -0.000106000 \\
\hline 6 & 14.969193000 & 2.175160000 & -0.000111000 \\
\hline 1 & 15.948419000 & 2.641537000 & -0.000132000 \\
\hline 6 & 15.935397000 & -1.432117000 & 0.000010000 \\
\hline 1 & 16.830529000 & -2.044028000 & 0.000027000 \\
\hline 6 & 14.678293000 & -2.046146000 & 0.000034000 \\
\hline 1 & 14.604266000 & -3.128315000 & 0.000068000 \\
\hline 6 & 13.824015000 & 2.945122000 & -0.000134000 \\
\hline 1 & 13.896946000 & 4.026959000 & -0.000176000 \\
\hline 1 & 9.753427000 & -2.881886000 & 0.000057000 \\
\hline 1 & 5.024707000 & 2.271894000 & 0.000019000 \\
\hline 1 & 11.688973000 & 2.985817000 & -0.000129000 \\
\hline 1 & -11.689113000 & -2.976064000 & -0.000156000 \\
\hline 1 & 4.811401000 & -2.709351000 & 0.000061000 \\
\hline 1 & 9.904676000 & 2.081939000 & -0.000029000 \\
\hline 6 & -6.176082000 & -0.464434000 & 0.000021000 \\
\hline 6 & -6.112956000 & 0.986005000 & 0.000032000 \\
\hline 6 & -7.441556000 & -1.091285000 & 0.000006000 \\
\hline 1 & -7.484830000 & -2.176268000 & -0.000004000 \\
\hline
\end{tabular}




$\begin{array}{lccc}6 & -4.977927000 & -1.198095000 & 0.000025000 \\ 6 & -4.855290000 & 1.611960000 & 0.000041000 \\ 6 & -8.562208000 & 1.088259000 & 0.000017000 \\ 6 & -8.622960000 & -0.361971000 & 0.000001000 \\ 6 & -7.319830000 & 1.718349000 & 0.000031000 \\ 1 & -7.271846000 & 2.802935000 & 0.000042000 \\ 6 & -9.786580000 & 1.803493000 & 0.000020000 \\ 6 & -9.904035000 & -0.994492000 & -0.000022000 \\ 1 & -9.753971000 & 2.888612000 & 0.000040000 \\ 1 & -5.026456000 & -2.282669000 & 0.000020000 \\ 1 & -4.807641000 & 2.696550000 & 0.000047000 \\ 1 & -9.907030000 & -2.077833000 & -0.000033000 \\ 6 & 1.192180000 & -0.793888000 & 0.000049000 \\ 6 & 1.254630000 & 0.668254000 & 0.000045000 \\ 6 & 3.716391000 & 0.562730000 & 0.000038000 \\ 6 & 3.656195000 & -0.897505000 & 0.000048000 \\ 6 & 2.521496000 & 1.290700000 & 0.000039000 \\ 6 & 2.396968000 & -1.522703000 & 0.000053000 \\ 1 & 2.567519000 & 2.375420000 & 0.000035000 \\ 1 & 2.351220000 & -2.607382000 & 0.000058000 \\ 6 & 0.065423000 & 1.397305000 & 0.000046000 \\ 6 & -0.058411000 & -1.417523000 & 0.000049000 \\ 1 & 0.109805000 & 2.482044000 & 0.000045000 \\ 1 & -0.108833000 & -2.501944000 & 0.000051000 \\ 6 & -3.726936000 & -0.573477000 & 0.000036000 \\ 6 & -3.662864000 & 0.880036000 & 0.000042000 \\ 6 & -1.206813000 & 0.774343000 & 0.000046000 \\ 6 & -12.157388000 & 2.983295000 & -0.000207000 \\ 6 & -1.270589000 & -0.685071000 & 0.000045000 \\ 6 & -11.032894000 & 1.157624000 & -0.000028000 \\ 6 & -2.393235000 & 1.504992000 & 0.000046000 \\ 6 & -2.516433000 & -1.308874000 & 0.000039000 \\ 1 & -2.348643000 & 2.589683000 & 0.000049000 \\ 1 & -2.568334000 & -2.393200000 & 0.000037000 \\ 6 & -13.587719000 & -0.143920000 & 0.000068000 \\ 6 & -1.089845000000 & -0.9498298000 & 0.000178000 \\ 6 & -1.291563000 & -0.000094000\end{array}$




\begin{tabular}{|c|c|c|c|}
\hline 6 & 4.852910000 & -1.617099000 & -0.000112000 \\
\hline 6 & -14.879185000 & -0.756122000 & 0.000139000 \\
\hline 6 & -16.040575000 & 0.065423000 & 0.000017000 \\
\hline 1 & -17.015422000 & -0.409667000 & 0.000060000 \\
\hline 6 & -12.562908000 & -2.338646000 & 0.000404000 \\
\hline 6 & -14.971778000 & -2.166488000 & 0.000341000 \\
\hline 1 & -15.951519000 & -2.631759000 & 0.000394000 \\
\hline 6 & -15.933029000 & 1.442383000 & -0.000153000 \\
\hline 1 & -16.827592000 & 2.055173000 & -0.000241000 \\
\hline 6 & -14.675591000 & 2.054531000 & -0.000204000 \\
\hline 1 & -14.599317000 & 3.136579000 & -0.000321000 \\
\hline 6 & -13.827572000 & -2.938111000 & 0.000480000 \\
\hline 1 & -13.902212000 & -4.019837000 & 0.000653000 \\
\hline 6 & 11.089845000 & 0.298764000 & 0.000070000 \\
\hline 6 & 8.571703000 & -1.086046000 & -0.000058000 \\
\hline 6 & 8.628511000 & 0.364684000 & -0.000008000 \\
\hline 6 & 13.587720000 & 0.143919000 & 0.000084000 \\
\hline 6 & 12.410148000 & 0.949224000 & 0.000172000 \\
\hline 6 & 11.032894000 & -1.157624000 & -0.000027000 \\
\hline 6 & 7.313092000 & -1.718414000 & -0.000094000 \\
\hline 1 & 7.270995000 & -2.803237000 & -0.000125000 \\
\hline 6 & 9.777726000 & -1.797460000 & -0.000075000 \\
\hline 6 & 12.218851000 & -1.899657000 & -0.000089000 \\
\hline 1 & 12.157386000 & -2.983295000 & -0.000170000 \\
\hline 6 & 13.495955000 & -1.291563000 & -0.000056000 \\
\hline 6 & 9.903677000 & 0.997973000 & 0.000054000 \\
\hline 6 & 14.879187000 & 0.756119000 & 0.000152000 \\
\hline 6 & 16.040577000 & -0.065427000 & 0.000056000 \\
\hline 1 & 17.015424000 & 0.409662000 & 0.000102000 \\
\hline 6 & 12.562915000 & 2.338647000 & 0.000361000 \\
\hline 6 & 14.971785000 & 2.166484000 & 0.000321000 \\
\hline 1 & 15.951528000 & 2.631751000 & 0.000371000 \\
\hline 6 & 15.933030000 & -1.442387000 & -0.000091000 \\
\hline 1 & 16.827591000 & -2.055180000 & -0.000162000 \\
\hline 6 & 14.675589000 & -2.054533000 & -0.000142000 \\
\hline 1 & 14.599318000 & -3.136581000 & -0.000245000 \\
\hline 6 & 13.827581000 & 2.938109000 & 0.000431000 \\
\hline 1 & 13.902222000 & 4.019835000 & 0.000575000 \\
\hline 1 & 9.748771000 & -2.882700000 & -0.000131000 \\
\hline 1 & 5.029014000 & 2.278069000 & -0.000036000 \\
\hline 1 & 11.692435000 & 2.981552000 & 0.000467000 \\
\hline 1 & -11.692424000 & -2.981546000 & 0.000533000 \\
\hline 1 & 4.808394000 & -2.701858000 & -0.000133000 \\
\hline 1 & 9.907985000 & 2.081275000 & 0.000086000 \\
\hline 6 & -6.175665000 & -0.465141000 & -0.000072000 \\
\hline 6 & -6.115547000 & 0.990761000 & -0.000074000 \\
\hline
\end{tabular}




\begin{tabular}{|c|c|c|c|}
\hline 6 & -7.441739000 & -1.091526000 & -0.000046000 \\
\hline 1 & -7.486673000 & -2.176432000 & -0.000037000 \\
\hline 6 & -4.980903000 & -1.193415000 & -0.000089000 \\
\hline 6 & -4.852911000 & 1.617099000 & -0.000083000 \\
\hline 6 & -8.571704000 & 1.086050000 & -0.000045000 \\
\hline 6 & -8.628512000 & -0.364680000 & -0.000022000 \\
\hline 6 & -7.313093000 & 1.718417000 & -0.000065000 \\
\hline 1 & -7.270999000 & 2.803240000 & -0.000074000 \\
\hline 6 & -9.777725000 & 1.797462000 & -0.000059000 \\
\hline 6 & -9.903678000 & -0.997973000 & 0.000032000 \\
\hline 1 & -9.748784000 & 2.882703000 & -0.000097000 \\
\hline 1 & -5.029026000 & -2.278066000 & -0.000089000 \\
\hline 1 & -4.808397000 & 2.701858000 & -0.000081000 \\
\hline 1 & -9.907979000 & -2.081274000 & 0.000048000 \\
\hline 6 & 1.197503000 & -0.782343000 & -0.000112000 \\
\hline 6 & 1.261395000 & 0.675787000 & -0.000096000 \\
\hline 6 & 3.719378000 & 0.569537000 & -0.000083000 \\
\hline 6 & 3.656772000 & -0.887872000 & -0.000106000 \\
\hline 6 & 2.522301000 & 1.298762000 & -0.000082000 \\
\hline 6 & 2.394152000 & -1.512155000 & -0.000119000 \\
\hline 1 & 2.571234000 & 2.383395000 & -0.000064000 \\
\hline 1 & 2.348289000 & -2.596917000 & -0.000133000 \\
\hline 6 & 0.064216000 & 1.405423000 & -0.000090000 \\
\hline 6 & -0.064216000 & -1.405426000 & -0.000118000 \\
\hline 1 & 0.112008000 & 2.490146000 & -0.000077000 \\
\hline 1 & -0.111999000 & -2.490149000 & -0.000126000 \\
\hline 6 & -3.719381000 & -0.569537000 & -0.000099000 \\
\hline 6 & -3.656773000 & 0.887872000 & -0.000091000 \\
\hline 6 & -1.197506000 & 0.782342000 & -0.000098000 \\
\hline 6 & -1.261401000 & -0.675788000 & -0.000111000 \\
\hline 6 & -2.394152000 & 1.512153000 & -0.000090000 \\
\hline 6 & -2.522305000 & -1.298761000 & -0.000111000 \\
\hline 1 & -2.348283000 & 2.596915000 & -0.000082000 \\
\hline 1 & -2.571235000 & -2.383394000 & -0.000114000 \\
\hline \multicolumn{4}{|c|}{$\mathrm{Q} \mathbf{1}(n=5) \alpha \beta \beta \alpha$} \\
\hline 6 & -11.027322000 & 1.155041000 & -0.000057000 \\
\hline 6 & 6.178981000 & 0.475046000 & -0.000185000 \\
\hline 6 & 6.118510000 & -0.978328000 & -0.000354000 \\
\hline 6 & -13.587562000 & -0.139885000 & 0.000326000 \\
\hline 6 & -12.413248000 & -0.948558000 & 0.000596000 \\
\hline 6 & -11.089293000 & -0.301903000 & 0.000241000 \\
\hline 6 & 7.444346000 & 1.101056000 & -0.000020000 \\
\hline 1 & 7.489834000 & 2.185931000 & 0.000121000 \\
\hline 6 & 4.981765000 & 1.203639000 & -0.000186000 \\
\hline 6 & -12.214502000 & 1.900738000 & -0.000206000 \\
\hline
\end{tabular}




\begin{tabular}{|c|c|c|c|}
\hline & -12.149918000 & 2.984149000 & -0.000444000 \\
\hline 6 & -13.491121000 & 1.296388000 & -0.000096000 \\
\hline 6 & 4.844233000 & -1.603684000 & -0.000459000 \\
\hline 6 & -14.880807000 & -0.747786000 & 0.000512000 \\
\hline 6 & -16.040123000 & 0.077390000 & 0.000222000 \\
\hline 1 & -17.016296000 & -0.394925000 & 0.000363000 \\
\hline 6 & -12.569984000 & -2.336974000 & 0.001134000 \\
\hline 6 & -14.978083000 & -2.157570000 & 0.001011000 \\
\hline 1 & -15.959346000 & -2.619624000 & 0.001149000 \\
\hline 6 & -15.928522000 & 1.454190000 & -0.000209000 \\
\hline 1 & -16.821342000 & 2.069519000 & -0.000437000 \\
\hline 6 & -14.669698000 & 2.062713000 & -0.000353000 \\
\hline 1 & -14.590393000 & 3.144526000 & -0.000667000 \\
\hline 6 & -13.836468000 & -2.932842000 & 0.001327000 \\
\hline 1 & -13.914307000 & -4.014332000 & 0.001731000 \\
\hline 6 & 11.089291000 & 0.301900000 & 0.000205000 \\
\hline 6 & 8.568625000 & -1.076576000 & -0.000250000 \\
\hline 6 & 8.628950000 & 0.372366000 & -0.000024000 \\
\hline 6 & 13.587562000 & 0.139874000 & 0.000323000 \\
\hline 6 & 12.413250000 & 0.948552000 & 0.000518000 \\
\hline 6 & 11.027316000 & -1.155051000 & -0.000132000 \\
\hline 6 & 7.307524000 & -1.705718000 & -0.000399000 \\
\hline 1 & 7.263319000 & -2.790557000 & -0.000550000 \\
\hline 6 & 9.773939000 & -1.790790000 & -0.000322000 \\
\hline 6 & 12.214499000 & -1.900747000 & -0.000296000 \\
\hline 1 & 12.149915000 & -2.984158000 & -0.000579000 \\
\hline 6 & 13.491120000 & -1.296398000 & -0.000115000 \\
\hline 6 & 9.907273000 & 1.003635000 & 0.000199000 \\
\hline 6 & 14.880810000 & 0.747770000 & 0.000590000 \\
\hline 6 & 16.040124000 & -0.077409000 & 0.000371000 \\
\hline 1 & 17.016298000 & 0.394905000 & 0.000569000 \\
\hline 6 & 12.569995000 & 2.336966000 & 0.001030000 \\
\hline 6 & 14.978093000 & 2.157554000 & 0.001077000 \\
\hline 1 & 15.959358000 & 2.619602000 & 0.001285000 \\
\hline 6 & 15.928521000 & -1.454206000 & -0.000082000 \\
\hline 1 & 16.821337000 & -2.069540000 & -0.000248000 \\
\hline 6 & 14.669691000 & -2.062727000 & -0.000318000 \\
\hline 1 & 14.590387000 & -3.144539000 & -0.000650000 \\
\hline 6 & 13.836482000 & 2.932830000 & 0.001301000 \\
\hline 1 & 13.914324000 & 4.014319000 & 0.001693000 \\
\hline 1 & 9.741886000 & -2.875953000 & -0.000544000 \\
\hline 1 & 5.028954000 & 2.288351000 & -0.000092000 \\
\hline 1 & 11.701456000 & 2.982548000 & 0.001244000 \\
\hline 1 & -11.701441000 & -2.982549000 & 0.001441000 \\
\hline 1 & 4.799460000 & -2.688549000 & -0.000567000 \\
\hline 1 & 9.913564000 & 2.086916000 & 0.000368000 \\
\hline
\end{tabular}




$\begin{array}{rrrr}6 & -6.178989000 & -0.475041000 & -0.000161000 \\ 6 & -6.118525000 & 0.978336000 & -0.000332000 \\ 6 & -7.444344000 & -1.101052000 & 0.000016000 \\ 1 & -7.489835000 & -2.185926000 & 0.000148000 \\ 6 & -4.981767000 & -1.203629000 & -0.000174000 \\ 6 & -4.844227000 & 1.603695000 & -0.000443000 \\ 6 & -8.568640000 & 1.076573000 & -0.000210000 \\ 6 & -8.628958000 & -0.372362000 & 0.000012000 \\ 6 & -7.307517000 & 1.705719000 & -0.000370000 \\ 1 & -7.263322000 & 2.790558000 & -0.000522000 \\ 6 & -9.773936000 & 1.790784000 & -0.000261000 \\ 6 & -9.907270000 & -1.003638000 & 0.000216000 \\ 1 & -9.741894000 & 2.875947000 & -0.000504000 \\ 1 & -5.028959000 & -2.288341000 & -0.000082000 \\ 1 & -4.799461000 & 2.688561000 & -0.000549000 \\ 1 & -9.913556000 & -2.086920000 & 0.000366000 \\ 6 & 1.202730000 & -0.782150000 & -0.000432000 \\ 6 & 1.263053000 & 0.682743000 & -0.000388000 \\ 6 & 3.724461000 & 0.579385000 & -0.000306000 \\ 6 & 3.664322000 & -0.879145000 & -0.000419000 \\ 6 & 2.523682000 & 1.305995000 & -0.000319000 \\ 6 & 2.384416000 & -1.504303000 & -0.000472000 \\ 1 & 2.570982000 & 2.390708000 & -0.000277000 \\ 1 & 2.339896000 & -2.589151000 & -0.000538000 \\ 6 & 0.070105000 & 1.406009000 & -0.000415000 \\ 6 & -0.070118000 & -1.405989000 & -0.000426000 \\ 1 & 0.116100000 & 2.490780000 & -0.000426000 \\ 1 & -0.116102000 & -2.490759000 & -0.000448000 \\ 6 & -3.724460000 & -0.579379000 & -0.000298000 \\ 6 & -3.664323000 & 0.879167000 & -0.000407000 \\ 6 & -1.202715000 & 0.782174000 & -0.000425000 \\ 6 & -1.263038000 & -0.682728000 & -0.000388000 \\ 6 & -2.384418000 & 1.504323000 & -0.000459000 \\ 6 & -2.523693000 & -1.305979000 & -0.000319000 \\ 1 & -2.339894000 & 2.589171000 & -0.000520000 \\ 1 & -2.570989000 & -2.390691000 & -0.000287000\end{array}$

\begin{tabular}{|c|c|c|c|}
\hline \multicolumn{4}{|c|}{$\operatorname{css} 2(n=1)$} \\
\hline 6 & 6.106026000 & -0.739393000 & 0.000002000 \\
\hline 6 & 3.677301000 & 0.730936000 & 0.000003000 \\
\hline 6 & 3.677254000 & -0.730837000 & 0.000001000 \\
\hline 6 & 9.664255000 & 0.000015000 & 0.000005000 \\
\hline 6 & 8.268379000 & 0.000003000 & 0.000005000 \\
\hline 6 & 6.106072000 & 0.739434000 & 0.000004000 \\
\hline & 7.484037000 & 1.177183000 & 0.000006000 \\
\hline
\end{tabular}




$\begin{array}{cccc}6 & 2.461364000 & 1.404733000 & 0.000003000 \\ 1 & 2.460855000 & 2.490737000 & 0.000004000 \\ 6 & 4.920579000 & 1.428380000 & 0.000004000 \\ 6 & 9.541959000 & 2.445691000 & 0.000008000 \\ 1 & 10.052309000 & 3.403122000 & 0.000010000 \\ 6 & 10.340807000 & 1.252583000 & 0.000007000 \\ 6 & 4.920468000 & -1.428368000 & 0.000001000 \\ 6 & 10.340814000 & -1.252618000 & 0.000004000 \\ 6 & 11.749319000 & -1.221580000 & 0.000005000 \\ 1 & 12.310441000 & -2.149984000 & 0.000004000 \\ 6 & 8.157090000 & 2.417118000 & 0.000008000 \\ 1 & 7.605859000 & 3.351576000 & 0.000008000 \\ 6 & 7.483974000 & -1.177103000 & 0.000003000 \\ 6 & 9.541806000 & -2.445766000 & 0.000002000 \\ 1 & 10.052173000 & -3.403189000 & 0.000001000 \\ 6 & 12.425620000 & -0.000003000 & 0.000007000 \\ 1 & 13.510251000 & -0.000124000 & 0.000008000 \\ 6 & 11.749459000 & 1.221468000 & 0.000008000 \\ 1 & 12.310475000 & 2.149936000 & 0.000010000 \\ 6 & 8.157010000 & -2.417166000 & 0.000001000 \\ 1 & 7.605702000 & -3.351567000 & 0.000000000 \\ 6 & 1.224483000 & 0.728067000 & 0.000002000 \\ 6 & -9.664256000 & -0.000016000 & -0.000005000 \\ 6 & -8.268378000 & -0.000004000 & -0.000005000 \\ 6 & 1.224422000 & -0.728029000 & 0.000000000 \\ 6 & -7.484037000 & -1.177185000 & -0.000006000 \\ 6 & 2.461277000 & -1.404705000 & 0.000000000 \\ 1 & 2.460673000 & -2.490700000 & -0.000002000 \\ 6 & -9.541961000 & -2.445695000 & -0.000009000 \\ 1 & -10.052311000 & -3.403127000 & -0.000010000 \\ 6 & -10.340809000 & -1.252585000 & -0.000007000 \\ 6 & -10.340817000 & 1.252619000 & -0.000004000 \\ 6 & -11.749322000 & 1.221580000 & -0.000005000 \\ 1 & -12.310445000 & 2.149985000 & -0.000004000 \\ 6 & -8.157090000 & -2.417121000 & -0.000008000 \\ 1 & -7.605858000 & -3.351579000 & -0.000009000 \\ 6 & -7.483974000 & 1.177105000 & -0.000003000 \\ 6 & -9.541808000 & 2.445768000 & -0.000002000 \\ 1 & -10.052176000 & 3.403192000 & -0.000001000 \\ 6 & -12.425623000 & 0.000002000 & -0.000007000 \\ 6 & -13.510254000 & 0.000123000 & -0.000008000 \\ 6 & -1.749462000 & -1.221470000 & -0.000008000 \\ 6 & -310479000 & -2.149938000 & -0.000010000 \\ 6 & -157011000 & 2.417169000 & -0.000001000 \\ 6 & -2.351570000 & 0.000000000 \\ 6 & -513804000 & -0.000001000\end{array}$




\begin{tabular}{|c|c|c|c|}
\hline & 4.906421000 & 2.513833000 & \\
\hline & -3.677300000 & -0.730937000 & -0.000003000 \\
\hline & -3.677252000 & 0.730839000 & -0.000001000 \\
\hline & -4.920578000 & -1.428382000 & -0.000004000 \\
\hline 1 & -4.906420000 & -2.513836000 & -0.000006000 \\
\hline & -2.461362000 & -1.404735000 & -0.000003000 \\
\hline & -2.461274000 & 1.40470 & 0.000001000 \\
\hline & -6.106026000 & 0.739395000 & -0.000002000 \\
\hline 0 & -6.106071000 & -0.739435000 & -0.000004000 \\
\hline 0 & -4.920467000 & 1.428370000 & -0.000001000 \\
\hline & -4.906120000 & 2.51380 & 0.000001000 \\
\hline & -2.460852000 & -2.490740000 & -0.000004000 \\
\hline & -2.460670000 & 2.490705000 & 0.000002000 \\
\hline 6 & -0.000041000 & -1.406912000 & -0.000002000 \\
\hline & 0.000044000 & 1.406913000 & 0.000002000 \\
\hline & -0.000080 & -2.4 & -0.000003000 \\
\hline & 0.000 & 2.4 & 0.000003000 \\
\hline 6 & -1.22 & 0.72 & 0.000000000 \\
\hline & -1.224481000 & -0.728067000 & -0.000002000 \\
\hline \multicolumn{4}{|c|}{ oss $2(n=1)$} \\
\hline 6 & 6.105833000 & -0.736 & -0.000 \\
\hline & 3.675771000 & 0.72 & -0.000002000 \\
\hline & 3.67 & -0.7 & -0.0 \\
\hline 0 & 9.67 & 0.00 & 0.000 \\
\hline 6 & 8.280802000 & 0.00 & 3000 \\
\hline & 6.105815000 & 0.736 & \\
\hline 6 & 7.49 & 1.17 & 3000 \\
\hline 6 & 2.46 & 1.4 & -0.00 \\
\hline 1 & 2.468 & 2.4 & 1000 \\
\hline & & & \\
\hline & 9.556 & 2.4 & 2000 \\
\hline 1 & 10.064024000 & 5000 & 15000 \\
\hline 6 & 10.352424000 & 1.252 & 12000 \\
\hline & & -1.428 & -0.000003000 \\
\hline & 10.352445000 & 5000 & 0.000009000 \\
\hline & 11.764245000 & 97000 & 0.000014000 \\
\hline 1 & 12.324779000 & -2.1500 & 15000 \\
\hline & 8.164425000 & 2.412026000 & 0.000008000 \\
\hline & 7.613826000 & 3.346720000 & 0.000008000 \\
\hline & 7.496941000 & -1.177077000 & 0.000000000 \\
\hline 6 & 9.556137000 & -2.441810000 & 0.000006000 \\
\hline & 10.064089000 & -3.400524000 & 0.000007000 \\
\hline & 12.440630000 & 0.000054000 & 0.000018000 \\
\hline & 13.525242000 & 0.000018000 & 0.000022000 \\
\hline & 11.764279000 & 1.221360000 & 0.000017000 \\
\hline
\end{tabular}




\begin{tabular}{|c|c|c|c|}
\hline 1 & 12.324758000 & 2.150136000 & 0.000021000 \\
\hline & 8.164467000 & -2.412074000 & 0.000002000 \\
\hline 1 & 7.613839000 & -3.346729000 & 0.000000000 \\
\hline 6 & 1.224089000 & 0.727176000 & -0.000003000 \\
\hline 6 & -9.675806000 & 0.000007000 & -0.000003000 \\
\hline 6 & -8.280955000 & -0.000004000 & -0.000006000 \\
\hline 6 & 1.224053000 & -0.727297000 & -0.000005000 \\
\hline 6 & -7.497094000 & -1.177053000 & -0.000007000 \\
\hline 6 & 2.465994000 & -1.404079000 & -0.000005000 \\
\hline 1 & 2.468507000 & -2.489770000 & -0.000007000 \\
\hline 6 & -9.556284000 & -2.441693000 & -0.000005000 \\
\hline 1 & -10.064110000 & -3.400478000 & -0.000005000 \\
\hline 6 & -10.352464000 & -1.252855000 & -0.000003000 \\
\hline 6 & -10.352436000 & 1.252917000 & 0.000000000 \\
\hline 6 & -11.764293000 & 1.221367000 & 0.000003000 \\
\hline 1 & -12.324795000 & 2.150136000 & 0.000006000 \\
\hline 6 & -8.164390000 & -2.411905000 & -0.000007000 \\
\hline 1 & -7.613849000 & -3.346635000 & -0.000008000 \\
\hline 6 & -7.497069000 & 1.177033000 & -0.000004000 \\
\hline 6 & -9.556186000 & 2.441766000 & 0.000001000 \\
\hline 1 & -10.064025000 & 3.400544000 & 0.000004000 \\
\hline 6 & -12.440749000 & 0.000039000 & 0.000002000 \\
\hline 1 & -13.525347000 & 0.000106000 & 0.000005000 \\
\hline 6 & -11.764399000 & -1.221256000 & 0.000000000 \\
\hline 1 & -12.324889000 & -2.150028000 & 0.000000000 \\
\hline 6 & -8.164331000 & 2.411980000 & 0.000000000 \\
\hline 1 & -7.613724000 & 3.346649000 & 0.000001000 \\
\hline 1 & 4.919272000 & -2.513101000 & -0.000005000 \\
\hline 1 & 4.919325000 & 2.513110000 & 0.000001000 \\
\hline 6 & -3.676644000 & -0.728662000 & -0.000007000 \\
\hline 6 & -3.676670000 & 0.728589000 & -0.000005000 \\
\hline 6 & -4.928781000 & -1.427533000 & -0.000008000 \\
\hline 1 & -4.918664000 & -2.512677000 & -0.000009000 \\
\hline 6 & -2.465009000 & -1.404140000 & -0.000007000 \\
\hline 6 & -2.464946000 & 1.403985000 & -0.000004000 \\
\hline 6 & -6.105775000 & 0.735455000 & -0.000005000 \\
\hline 6 & -6.105766000 & -0.735454000 & -0.000007000 \\
\hline 6 & -4.928730000 & 1.427505000 & -0.000004000 \\
\hline 1 & -4.918558000 & 2.512635000 & -0.000003000 \\
\hline 1 & -2.469129000 & -2.489778000 & -0.000009000 \\
\hline 1 & -2.469123000 & 2.489624000 & -0.000002000 \\
\hline 6 & 0.000585000 & -1.405639000 & -0.000006000 \\
\hline 6 & 0.000508000 & 1.405414000 & -0.000003000 \\
\hline 1 & -0.001425000 & -2.491294000 & -0.000008000 \\
\hline 1 & -0.001739000 & 2.491084000 & -0.000002000 \\
\hline 6 & -1.224710000 & 0.726734000 & -0.000004000 \\
\hline
\end{tabular}




$$
\text { T2 }(n=1)
$$

$\begin{array}{lccc}6 & 6.106854000 & -0.736736000 & 0.000002000 \\ 6 & 3.676831000 & 0.729121000 & 0.000003000 \\ 6 & 3.676826000 & -0.729115000 & 0.000001000 \\ 6 & 9.676785000 & -0.000006000 & 0.000005000 \\ 6 & 8.281823000 & -0.000003000 & 0.000004000 \\ 6 & 6.106855000 & 0.736740000 & 0.000004000 \\ 6 & 7.498219000 & 1.176763000 & 0.000005000 \\ 6 & 2.465983000 & 1.404280000 & 0.000003000 \\ 1 & 2.465133000 & 2.490134000 & 0.000004000 \\ 6 & 4.930148000 & 1.427621000 & 0.000004000 \\ 6 & 9.557049000 & 2.442037000 & 0.000009000 \\ 1 & 10.065411000 & 3.400523000 & 0.000011000 \\ 6 & 10.353375000 & 1.252711000 & 0.000008000 \\ 6 & 4.930145000 & -1.427613000 & 0.000001000 \\ 6 & 10.353372000 & -1.252725000 & 0.000004000 \\ 6 & 11.765121000 & -1.221322000 & 0.000006000 \\ 1 & 12.325601000 & -2.150072000 & 0.000005000 \\ 6 & 8.165586000 & 2.412177000 & 0.000008000 \\ 1 & 7.614261000 & 3.346438000 & 0.000009000 \\ 6 & 7.498213000 & -1.176762000 & 0.000002000 \\ 6 & 9.557037000 & -2.442048000 & 0.000002000 \\ 1 & 10.065398000 & -3.400535000 & 0.000002000 \\ 6 & 12.441654000 & -0.000010000 & 0.000008000 \\ 1 & 13.526202000 & -0.000014000 & 0.000009000 \\ 6 & 11.765126000 & 1.221301000 & 0.000009000 \\ 1 & 12.325603000 & 2.150053000 & 0.000011000 \\ 6 & 8.165576000 & -2.412178000 & 0.000001000 \\ 1 & 7.614243000 & -3.346437000 & 0.000000000 \\ 6 & 1.224233000 & 0.726216000 & 0.000001000 \\ 6 & -9.676795000 & -0.000006000 & -0.000005000 \\ 6 & -8.281850000 & -0.000003000 & -0.000005000 \\ 6 & 1.224236000 & -0.726191000 & -0.000001000 \\ 6 & -7.498225000 & -1.176769000 & -0.000006000 \\ 6 & 2.465980000 & -1.404286000 & -0.000001000 \\ 1 & 2.465133000 & -2.490140000 & -0.000002000 \\ 6 & -9.557064000 & -2.442023000 & -0.000008000 \\ 1 & -10.065382000 & -3.400534000 & -0.000009000 \\ 6 & -10.353383000 & -1.252736000 & -0.000007000 \\ 6 & -10.353391000 & 1.252722000 & -0.000004000 \\ 6 & -11.765153000 & 1.221302000 & -0.000005000 \\ 1 & -12.325637000 & 2.150050000 & -0.000004000 \\ 6 & -8.165562000 & -2.412153000 & -0.000007000 \\ 1 & -7.614277000 & -3.346437000 & -0.000008000\end{array}$




\begin{tabular}{|c|c|c|c|}
\hline 6 & -7.498224000 & 1.176765000 & -0.000003000 \\
\hline 6 & -9.557068000 & 2.442014000 & -0.000002000 \\
\hline 1 & -10.065395000 & 3.400520000 & -0.000001000 \\
\hline 6 & -12.441678000 & -0.000014000 & -0.000006000 \\
\hline 1 & -13.526227000 & -0.000011000 & -0.000007000 \\
\hline 6 & -11.765152000 & -1.221319000 & -0.000008000 \\
\hline 1 & -12.325622000 & -2.150077000 & -0.000009000 \\
\hline 6 & -8.165571000 & 2.412145000 & -0.000001000 \\
\hline 1 & -7.614284000 & 3.346431000 & 0.000000000 \\
\hline 1 & 4.916949000 & -2.512948000 & -0.000001000 \\
\hline 1 & 4.916954000 & 2.512955000 & 0.000006000 \\
\hline 6 & -3.676854000 & -0.729044000 & -0.000003000 \\
\hline 6 & -3.676855000 & 0.729057000 & -0.000001000 \\
\hline 6 & -4.930095000 & -1.427584000 & -0.000005000 \\
\hline 1 & -4.917049000 & -2.512903000 & -0.000006000 \\
\hline 6 & -2.465842000 & -1.404238000 & -0.000003000 \\
\hline 6 & -2.465849000 & 1.404261000 & 0.000000000 \\
\hline 6 & -6.106844000 & 0.736641000 & -0.000003000 \\
\hline 6 & -6.106839000 & -0.736636000 & -0.000005000 \\
\hline 6 & -4.930098000 & 1.427591000 & -0.000001000 \\
\hline 1 & -4.917057000 & 2.512911000 & 0.000000000 \\
\hline 1 & -2.465167000 & -2.490086000 & -0.000005000 \\
\hline 1 & -2.465174000 & 2.490109000 & 0.000002000 \\
\hline 6 & 0.000091000 & -1.406193000 & -0.000002000 \\
\hline 6 & 0.000095000 & 1.406228000 & 0.000002000 \\
\hline 1 & 0.000077000 & -2.491923000 & -0.000003000 \\
\hline 1 & 0.000094000 & 2.491955000 & 0.000003000 \\
\hline 6 & -1.224395000 & 0.726191000 & 0.000000000 \\
\hline 6 & -1.224391000 & -0.726162000 & -0.000002000 \\
\hline \multicolumn{4}{|c|}{$\mathrm{Q} 2(n=1)$} \\
\hline 6 & 6.134918000 & -0.721769000 & 0.000000000 \\
\hline 6 & 3.710237000 & 0.718307000 & -0.000001000 \\
\hline 6 & 3.710370000 & -0.718304000 & -0.000002000 \\
\hline 6 & 9.708971000 & 0.000076000 & 0.000007000 \\
\hline 6 & 8.317470000 & 0.000056000 & 0.000003000 \\
\hline 6 & 6.134888000 & 0.721851000 & 0.000002000 \\
\hline 6 & 7.530326000 & 1.173721000 & 0.000003000 \\
\hline 6 & 2.462481000 & 1.399393000 & -0.000001000 \\
\hline 1 & 2.463957000 & 2.485244000 & 0.000001000 \\
\hline 6 & 4.940918000 & 1.416824000 & 0.000001000 \\
\hline 6 & 9.590069000 & 2.438522000 & 0.000010000 \\
\hline 1 & 10.095572000 & 3.398585000 & 0.000013000 \\
\hline 6 & 10.385738000 & 1.253302000 & 0.000010000 \\
\hline 6 & 4.940954000 & -1.416787000 & -0.000002000 \\
\hline 6 & 10.385762000 & -1.253156000 & 0.000008000 \\
\hline
\end{tabular}




\begin{tabular}{|c|c|c|c|}
\hline & 11.799577000 & -1.220844000 & \\
\hline & 12.359907000 & -2.149779000 & 0.000013000 \\
\hline & .193315000 & 2.406772000 & 0.000007000 \\
\hline & 7.642424000 & 3.341334000 & 0.000007000 \\
\hline & 7.530400000 & -1.173610000 & 01000 \\
\hline & 9.590120000 & -2.438399000 & \\
\hline & 10.0956 & -3.3 & 700 \\
\hline & 12.4769 & 0.000 & 1500 \\
\hline & 13.561390000 & 0.000082000 & 1800 \\
\hline & 11.7 & 1.22 & 0.0 \\
\hline & 12.3 & & 700 \\
\hline & & -2.40 & \\
\hline & 7.6424 & -3.341219000 & 02000 \\
\hline & 000 & 0.727 & \\
\hline & -9.70 & 0.00009 & -0.000 \\
\hline & -8.3 & 0.00 & -0.0 \\
\hline & 1.2 & -0.7 & -0.0 \\
\hline & -7.5 & -1.17 & 8000 \\
\hline & 2.462 & -1.39 & -0.00 \\
\hline & 2.46 & -2.4 & -0.0 \\
\hline & -9.58 & -2.438 & -0.00 \\
\hline & 000 & -3.3 & \\
\hline & -10.3 & -1.252 & -0.0 \\
\hline & -10.3 & 1.2 & 0.0 \\
\hline & -11.7 & 000 & 0.00 \\
\hline & -12.3 & 2.1 & 0.0 \\
\hline & -8.19 & -2.406 & -0.00 \\
\hline & -7.6 & -3.3 & -0.0 \\
\hline & -7.5 & 1.17 & -0.0 \\
\hline & -9.58 & 2.43 & 000 \\
\hline & & & \\
\hline & -12.4 & 0.00 & 000 \\
\hline & -13.5 & 0.00 & 0.0 \\
\hline & -11.7 & -1.22 & -0.0 \\
\hline & -12.35 & -2.12 & \\
\hline & -8.193 & 2.40 & \\
\hline & -7.64 & 3.34 & 0.000 \\
\hline & 4.92795 & -2.502 & -0.00 \\
\hline & 870000 & 2.502 & 0.00000200 \\
\hline & -3.710 & -0.718 & -0.00 \\
\hline & -3.710023000 & 0.718036000 & -0.00000400 \\
\hline & -4.941537000 & -1.417119000 & -0.00000700 \\
\hline & -4.928242000 & -2.502477000 & -0.00000800 \\
\hline & -2.462948000 & -1.399744000 & -0.00000600 \\
\hline & -2.462774000 & 1.399294000 & -0.00000300 \\
\hline & -6.134566000 & 0.722356000 & -0.00000400 \\
\hline
\end{tabular}




$\begin{array}{rrrr}6 & -6.134631000 & -0.722497000 & -0.000006000 \\ 6 & -4.941383000 & 1.416868000 & -0.000004000 \\ 1 & -4.927973000 & 2.502218000 & -0.000002000 \\ 1 & -2.465127000 & -2.485588000 & -0.000007000 \\ 1 & -2.464952000 & 2.485141000 & -0.000001000 \\ 6 & 0.000296000 & -1.406588000 & -0.000005000 \\ 6 & 0.000391000 & 1.406124000 & -0.000002000 \\ 1 & -0.001632000 & -2.492116000 & -0.000006000 \\ 1 & -0.001810000 & 2.491669000 & -0.000001000 \\ 6 & -1.242884000 & 0.726450000 & -0.000003000 \\ 6 & -1.242869000 & -0.726956000 & -0.000005000\end{array}$

$\begin{array}{lccc}\text { CsS } 2(n=2) & & \\ 6 & 7.329158000 & -0.736939000 & -0.000002000 \\ 6 & 4.903881000 & 0.731764000 & -0.000003000 \\ 6 & 4.902822000 & -0.728082000 & -0.000005000 \\ 6 & 10.888699000 & -0.000594000 & 0.000010000 \\ 6 & 9.493616000 & -0.001587000 & 0.000004000 \\ 6 & 7.330601000 & 0.736809000 & 0.000000000 \\ 6 & 8.709952000 & 1.177866000 & 0.000004000 \\ 6 & 3.686298000 & 1.407880000 & -0.000004000 \\ 1 & 3.690584000 & 2.493539000 & -0.000003000 \\ 6 & 6.146426000 & 1.429711000 & -0.000001000 \\ 6 & 10.767049000 & 2.445489000 & 0.000015000 \\ 1 & 11.275131000 & 3.404071000 & 0.000020000 \\ 6 & 11.564777000 & 1.253670000 & 0.000016000 \\ 6 & 6.147786000 & -1.428800000 & -0.000004000 \\ 6 & 11.565358000 & -1.254266000 & 0.000012000 \\ 6 & 12.976342000 & -1.220812000 & 0.000019000 \\ 1 & 13.537759000 & -2.148962000 & 0.000020000 \\ 6 & 9.379766000 & 2.416178000 & 0.000009000 \\ 1 & 8.830160000 & 3.351539000 & 0.000010000 \\ 6 & 8.713084000 & -1.179722000 & 0.0000000000 \\ 6 & 10.770192000 & -2.445654000 & 0.000008000 \\ 1 & 11.278345000 & -3.404129000 & 0.000009000 \\ 6 & 13.650303000 & 0.000581000 & 0.000024000 \\ 1 & 14.734950000 & 0.001584000 & 0.000029000 \\ 6 & 12.973702000 & 1.222782000 & 0.000023000 \\ 1 & 13.534117000 & 2.151571000 & 0.000027000 \\ 6 & 9.381516000 & -2.416993000 & 0.000002000 \\ 1 & 8.832295000 & -3.352624000 & 0.000000000 \\ 6 & -0.000026000 & 0.731288000 & -0.000006000 \\ 6 & 2.451246000 & -0.727637000 & -0.000007000 \\ 6 & 2.451583000 & 0.731006000 & -0.000005000 \\ 6 & -10.888699000 & 0.000594000 & -0.000002000\end{array}$




\begin{tabular}{|c|c|c|c|}
\hline 6 & -9.493615000 & 0.001587000 & -0.000005000 \\
\hline 6 & 0.000025000 & -0.731288000 & -0.000008000 \\
\hline 6 & -8.709952000 & -1.177866000 & -0.000007000 \\
\hline 6 & 3.688716000 & -1.404801000 & -0.000007000 \\
\hline 1 & 3.693163000 & -2.490464000 & -0.000008000 \\
\hline 6 & 1.230201000 & -1.407927000 & -0.000008000 \\
\hline 6 & -10.767048000 & -2.445491000 & -0.000003000 \\
\hline 1 & -11.275130000 & -3.404074000 & -0.000002000 \\
\hline 6 & -11.564777000 & -1.253672000 & -0.000001000 \\
\hline 6 & 1.226888000 & 1.409479000 & -0.000005000 \\
\hline 6 & -11.565358000 & 1.254268000 & 0.000001000 \\
\hline 6 & -12.976344000 & 1.220813000 & 0.000005000 \\
\hline 1 & -13.537761000 & 2.148963000 & 0.000008000 \\
\hline 6 & -9.379765000 & -2.416178000 & -0.000005000 \\
\hline 1 & -8.830158000 & -3.351539000 & -0.000006000 \\
\hline 6 & -8.713083000 & 1.179723000 & -0.000004000 \\
\hline 6 & -10.770193000 & 2.445655000 & 0.000002000 \\
\hline 1 & -11.278345000 & 3.404131000 & 0.000004000 \\
\hline 6 & -13.650304000 & -0.000581000 & 0.000006000 \\
\hline 1 & -14.734951000 & -0.001585000 & 0.000009000 \\
\hline 6 & -12.973703000 & -1.222783000 & 0.000003000 \\
\hline 1 & -13.534119000 & -2.151573000 & 0.000004000 \\
\hline 6 & -9.381515000 & 2.416994000 & -0.000001000 \\
\hline 1 & -8.832295000 & 3.352625000 & 0.000000000 \\
\hline 1 & 1.234918000 & -2.493552000 & -0.000010000 \\
\hline 1 & 6.134118000 & -2.513922000 & -0.000006000 \\
\hline 1 & 6.135266000 & 2.514819000 & 0.000000000 \\
\hline 1 & 1.228970000 & 2.495108000 & -0.000004000 \\
\hline 6 & -4.903881000 & -0.731762000 & -0.000008000 \\
\hline 6 & -4.902822000 & 0.728081000 & -0.000006000 \\
\hline 6 & -6.146426000 & -1.429710000 & -0.000008000 \\
\hline 1 & -6.135266000 & -2.514818000 & -0.000010000 \\
\hline 6 & -3.686298000 & -1.407879000 & -0.000009000 \\
\hline 6 & -3.688715000 & 1.404799000 & -0.000005000 \\
\hline 6 & -7.329157000 & 0.736938000 & -0.000005000 \\
\hline 6 & -7.330600000 & -0.736807000 & -0.000007000 \\
\hline 6 & -6.147785000 & 1.428800000 & -0.000004000 \\
\hline 1 & -6.134116000 & 2.513922000 & -0.000003000 \\
\hline 1 & -3.690585000 & -2.493537000 & -0.000010000 \\
\hline 1 & -3.693163000 & 2.490463000 & -0.000003000 \\
\hline 6 & -1.226888000 & -1.409479000 & -0.000009000 \\
\hline 6 & -1.230201000 & 1.407926000 & -0.000005000 \\
\hline 1 & -1.228970000 & -2.495108000 & -0.000010000 \\
\hline 1 & -1.234919000 & 2.493551000 & -0.000004000 \\
\hline 6 & -2.451247000 & 0.727636000 & -0.000006000 \\
\hline 6 & -2.451584000 & -0.731006000 & -0.000008000 \\
\hline
\end{tabular}




$\begin{array}{lccc}\text { oss } 2(n=2) & & \\ 6 & 7.335064000 & 0.738201000 & -0.000004000 \\ 6 & 4.903434000 & -0.729066000 & -0.000005000 \\ 6 & 4.904176000 & 0.732240000 & -0.000003000 \\ 6 & 10.903650000 & -0.001740000 & -0.000005000 \\ 6 & 9.508465000 & -0.001274000 & -0.000005000 \\ 6 & 7.334080000 & -0.737860000 & -0.000006000 \\ 6 & 8.725231000 & -1.177707000 & -0.000005000 \\ 6 & 3.695483000 & -1.403519000 & -0.000005000 \\ 1 & 3.693536000 & -2.489357000 & -0.000007000 \\ 6 & 6.158155000 & -1.427888000 & -0.000006000 \\ 6 & 10.783339000 & -2.443969000 & -0.000005000 \\ 1 & 11.291509000 & -3.402541000 & -0.000005000 \\ 6 & 11.579613000 & -1.254691000 & -0.000005000 \\ 6 & 6.158476000 & 1.429258000 & -0.000003000 \\ 6 & 11.580890000 & 1.250708000 & -0.000005000 \\ 6 & 12.991724000 & 1.219191000 & -0.000005000 \\ 1 & 13.552950000 & 2.147467000 & -0.000005000 \\ 6 & 9.392096000 & -2.413647000 & -0.000005000 \\ 1 & 8.840309000 & -3.347624000 & -0.000005000 \\ 6 & 8.724774000 & 1.176220000 & -0.000005000 \\ 6 & 10.784173000 & 2.441048000 & -0.000005000 \\ 1 & 11.293507000 & 3.399025000 & -0.000005000 \\ 6 & 13.668199000 & -0.002975000 & -0.000005000 \\ 1 & 14.752763000 & -0.002711000 & -0.000005000 \\ 6 & 12.991762000 & -1.223563000 & -0.000005000 \\ 1 & 13.551625000 & -2.152694000 & -0.000005000 \\ 6 & 9.393741000 & 2.411891000 & -0.000005000 \\ 1 & 8.842484000 & 3.346234000 & -0.000004000 \\ 6 & -0.000003000 & -0.725511000 & -0.000003000 \\ 6 & 2.450323000 & 0.730102000 & -0.000002000 \\ 6 & 2.449943000 & -0.725383000 & -0.000004000 \\ 6 & -10.903649000 & -0.001749000 & 0.000007000 \\ 6 & -9.508463000 & -0.001273000 & 0.000006000 \\ 6 & -0.000002000 & 0.730685000 & -0.000001000 \\ 6 & -8.724784000 & 1.176216000 & 0.000008000 \\ 6 & 3.695710000 & 1.407311000 & -0.000002000 \\ 1 & 3.694712000 & 2.493173000 & -0.000001000 \\ 6 & 1.231559000 & 1.410025000 & 0.000000000 \\ 6 & -10.784174000 & 2.441048000 & 0.000015000 \\ 1 & -11.293514000 & 3.399023000 & 0.000018000 \\ 6 & -11.580890000 & 1.250705000 & 0.000011000 \\ 6 & 1.231470000 & -1.405075000 & -0.000004000 \\ 6 & -11.579610000 & -1.254695000 & 0.000004000 \\ 6 & -12.991769000 & -1.223572000 & 0.000005000 \\ 1 & -13.551612000 & -2.152714000 & 0.000003000\end{array}$




\begin{tabular}{|c|c|c|c|}
\hline 6 & -9.393744000 & 2.411903000 & 0.000013000 \\
\hline 1 & -8.842485000 & 3.346240000 & 0.000015000 \\
\hline 6 & -8.725222000 & -1.177707000 & 0.000002000 \\
\hline 6 & -10.783335000 & -2.443970000 & 0.000000000 \\
\hline 1 & -11.291496000 & -3.402546000 & -0.000002000 \\
\hline 6 & -13.668198000 & -0.002988000 & 0.000009000 \\
\hline 1 & -14.752763000 & -0.002698000 & 0.000010000 \\
\hline 6 & -12.991715000 & 1.219183000 & 0.000012000 \\
\hline 1 & -13.552959000 & 2.147449000 & 0.000016000 \\
\hline 6 & -9.392089000 & -2.413635000 & -0.000001000 \\
\hline 1 & -8.840302000 & -3.347617000 & -0.000004000 \\
\hline 1 & 1.231281000 & 2.495705000 & 0.000000000 \\
\hline 1 & 6.146023000 & 2.514636000 & -0.000002000 \\
\hline 1 & 6.143821000 & -2.513207000 & -0.000007000 \\
\hline 1 & 1.231001000 & -2.490750000 & -0.000006000 \\
\hline 6 & -4.904188000 & 0.732246000 & 0.000003000 \\
\hline 6 & -4.903425000 & -0.729060000 & 0.000001000 \\
\hline 6 & -6.158476000 & 1.429269000 & 0.000005000 \\
\hline 1 & -6.145996000 & 2.514643000 & 0.000007000 \\
\hline 6 & -3.695704000 & 1.407305000 & 0.000003000 \\
\hline 6 & -3.695489000 & -1.403525000 & -0.000001000 \\
\hline 6 & -7.334077000 & -0.737851000 & 0.000003000 \\
\hline 6 & -7.335069000 & 0.738212000 & 0.000005000 \\
\hline 6 & -6.158154000 & -1.427877000 & 0.000001000 \\
\hline 1 & -6.143842000 & -2.513200000 & -0.000001000 \\
\hline 1 & -3.694733000 & 2.493168000 & 0.000004000 \\
\hline 1 & -3.693514000 & -2.489362000 & -0.000002000 \\
\hline 6 & -1.231550000 & 1.410018000 & 0.000001000 \\
\hline 6 & -1.231482000 & -1.405081000 & -0.000003000 \\
\hline 1 & -1.231325000 & 2.495701000 & 0.000002000 \\
\hline 1 & -1.230972000 & -2.490753000 & -0.000004000 \\
\hline 6 & -2.449941000 & -0.725366000 & -0.000001000 \\
\hline 6 & -2.450327000 & 0.730115000 & 0.000001000 \\
\hline \multicolumn{4}{|c|}{$\mathrm{T} 2(n=2)$} \\
\hline 6 & 7.335064000 & 0.738201000 & -0.000004000 \\
\hline 6 & 4.903434000 & -0.729066000 & -0.000005000 \\
\hline 6 & 4.904176000 & 0.732240000 & -0.000003000 \\
\hline 6 & 10.903650000 & -0.001740000 & -0.000005000 \\
\hline 6 & 9.508465000 & -0.001274000 & -0.000005000 \\
\hline 6 & 7.334080000 & -0.737860000 & -0.000006000 \\
\hline 6 & 8.725231000 & -1.177707000 & -0.000005000 \\
\hline 6 & 3.695483000 & -1.403519000 & -0.000005000 \\
\hline 1 & 3.693536000 & -2.489357000 & -0.000007000 \\
\hline 6 & 6.158155000 & -1.427888000 & -0.000006000 \\
\hline 6 & 10.783339000 & -2.443969000 & -0.000005000 \\
\hline
\end{tabular}




\begin{tabular}{|c|c|c|c|}
\hline & 1.291509000 & -3.402541000 & \\
\hline & 11.579613000 & -1.254691000 & \\
\hline & 6.158476000 & 1.429258000 & -0.000003000 \\
\hline & 11.580890000 & 1.250708000 & -0.00000500 \\
\hline & 12.991724000 & 1.219191000 & \\
\hline & 0000 & 67000 & \\
\hline & 9.392096000 & -2.41 & \\
\hline & 8.840309000 & -3.347624000 & -0.00 \\
\hline & 8.72 & 1.1762 & \\
\hline & 10.7 & & -0 \\
\hline & 11.2 & & \\
\hline & 13.6 & -0.00 & \\
\hline & 14.7 & -0.0 & \\
\hline & 12.99 & 3000 & -0.0 \\
\hline & 13.5 & -2.1 & \\
\hline & 9.35 & 2.41 & -0.0 \\
\hline & 8.8 & 3.3 & \\
\hline & 0003000 & -0.72 & -0.00 \\
\hline & 2.45 & 0.73 & -0.0 \\
\hline & 2.44 & -0.72 & -0.00 \\
\hline & -10.9 & -0.0 & \\
\hline & -9.50 & -0.00 & \\
\hline & -0.0 & 0.73 & -0.0 \\
\hline & -8.7 & 1.17 & 0.0 \\
\hline & 3.6 & 1.40 & -0.0 \\
\hline & & & \\
\hline & 1.23 & 1.41 & 0.0 \\
\hline & 000 & 2.44 & 0.0 \\
\hline & -11.2 & 3.39 & 0.0 \\
\hline & -11.5 & & \\
\hline & 1.23 & -1.40 & -0.0 \\
\hline & -11.5 & -1.25 & 0.00 \\
\hline & -12.9 & -1.22 & 0.0 \\
\hline & -13.5 & -2.1527 & \\
\hline & -9.39 & 2.4119 & \\
\hline & -8.8 & 3.34 & \\
\hline & -8.72 & -1.177 & 0.00 \\
\hline & -10.7 & -2.443 & 0.00000000 \\
\hline & -11.2 & -3.402 & -0.0000020 \\
\hline & -13.6 & -0.002 & 0.0000090 \\
\hline & -14.752763000 & -0.002698000 & 0.0000100 \\
\hline & -12.991715000 & 1.2191 & 0.00001200 \\
\hline & -13.552959000 & 2.147449000 & 0.00001600 \\
\hline & -9.392089000 & -2.413635000 & -0.00000100 \\
\hline & -8.840302000 & -3.347617000 & -0.00000400 \\
\hline & 1.231281000 & 2.495705000 & 0.000000000 \\
\hline
\end{tabular}




$\begin{array}{rrrr}1 & 6.146023000 & 2.514636000 & -0.000002000 \\ 1 & 6.143821000 & -2.513207000 & -0.000007000 \\ 1 & 1.231001000 & -2.490750000 & -0.000006000 \\ 6 & -4.904188000 & 0.732246000 & 0.000003000 \\ 6 & -4.903425000 & -0.729060000 & 0.000001000 \\ 6 & -6.158476000 & 1.429269000 & 0.000005000 \\ 1 & -6.145996000 & 2.514643000 & 0.000007000 \\ 6 & -3.695704000 & 1.407305000 & 0.000003000 \\ 6 & -3.695489000 & -1.403525000 & -0.000001000 \\ 6 & -7.334077000 & -0.737851000 & 0.000003000 \\ 6 & -7.335069000 & 0.738212000 & 0.000005000 \\ 6 & -6.158154000 & -1.427877000 & 0.000001000 \\ 1 & -6.143842000 & -2.513200000 & -0.000001000 \\ 1 & -3.694733000 & 2.493168000 & 0.000004000 \\ 1 & -3.693514000 & -2.489362000 & -0.000002000 \\ 6 & -1.231550000 & 1.410018000 & 0.000001000 \\ 6 & -1.231482000 & -1.405081000 & -0.000003000 \\ 1 & -1.231325000 & 2.495701000 & 0.000002000 \\ 1 & -1.230972000 & -2.490753000 & -0.000004000 \\ 6 & -2.449941000 & -0.725366000 & -0.000001000 \\ 6 & -2.450327000 & 0.730115000 & 0.000001000\end{array}$

$\begin{array}{lccc}\mathrm{Q} 2 & (n=2) \\ 6 & -7.360961000 & 0.725657000 & -0.000012000 \\ 6 & -4.935742000 & -0.721358000 & -0.000017000 \\ 6 & -4.934947000 & 0.718415000 & -0.000018000 \\ 6 & -10.935645000 & 0.000485000 & 0.000018000 \\ 6 & -9.542874000 & 0.000640000 & 0.000002000 \\ 6 & -7.361620000 & -0.726228000 & -0.000008000 \\ 6 & -8.757128000 & -1.174487000 & 0.000001000 \\ 6 & -3.696486000 & -1.400376000 & -0.000019000 \\ 1 & -3.697568000 & -2.486080000 & -0.000021000 \\ 6 & -6.173658000 & -1.420218000 & -0.000011000 \\ 6 & -10.816668000 & -2.439047000 & 0.000030000 \\ 1 & -11.323282000 & -3.398182000 & 0.000041000 \\ 6 & -11.612451000 & -1.252111000 & 0.000032000 \\ 6 & -6.172679000 & 1.418420000 & -0.000018000 \\ 6 & -11.612390000 & 1.253438000 & 0.000024000 \\ 6 & -13.025501000 & 1.221643000 & 0.000041000 \\ 1 & -13.586655000 & 2.149791000 & 0.000046000 \\ 6 & -9.421159000 & -2.408100000 & 0.000015000 \\ 1 & -8.870141000 & -3.342210000 & 0.000016000 \\ 6 & -8.756825000 & 1.173992000 & -0.000005000 \\ 6 & -10.815734000 & 2.439865000 & 0.000015000 \\ 1 & -11.321841000 & 3.399317000 & 0.000020000 \\ 6 & -13.703046000 & 0.000548000 & 0.000053000\end{array}$




$\begin{array}{cccc}1 & -14.787231000 & 0.001550000 & 0.000066000 \\ 6 & -13.025739000 & -1.220290000 & 0.000049000 \\ 1 & -13.585235000 & -2.149458000 & 0.000060000 \\ 6 & -9.420477000 & 2.408093000 & 0.000001000 \\ 1 & -8.869341000 & 3.342269000 & -0.000003000 \\ 6 & 0.000732000 & -0.731607000 & -0.000019000 \\ 6 & -2.476775000 & 0.723404000 & -0.000019000 \\ 6 & -2.476151000 & -0.726326000 & -0.000020000 \\ 6 & 10.935645000 & -0.000484000 & 0.000005000 \\ 6 & 9.542874000 & -0.000640000 & -0.000005000 \\ 6 & -0.000734000 & 0.731607000 & -0.000020000 \\ 6 & 8.757128000 & 1.174487000 & -0.000007000 \\ 6 & -3.695458000 & 1.397590000 & -0.000018000 \\ 1 & -3.696600000 & 2.483216000 & -0.000019000 \\ 6 & -1.227786000 & 1.405122000 & -0.000020000 \\ 6 & 10.816668000 & 2.439048000 & 0.000010000 \\ 1 & 11.323282000 & 3.398182000 & 0.000017000 \\ 6 & 11.612452000 & 1.252111000 & 0.000012000 \\ 6 & -1.228916000 & -1.406327000 & -0.000021000 \\ 6 & 11.612390000 & -1.253437000 & 0.000012000 \\ 6 & 13.025502000 & -1.221643000 & 0.000023000 \\ 1 & 13.586656000 & -2.149790000 & 0.000029000 \\ 6 & 9.421160000 & 2.408100000 & 0.000001000 \\ 1 & 8.870140000 & 3.342210000 & 0.000002000 \\ 6 & 8.756826000 & -1.173993000 & -0.000006000 \\ 6 & 10.815735000 & -2.439865000 & 0.000009000 \\ 6 & 11.321842000 & -3.399316000 & 0.000015000 \\ 6 & 13.703047000 & -0.000548000 & 0.000029000 \\ 6 & 6.17 .361620000 & 0.726228000 & -0.000009000 \\ 1 & 14.787231000 & -0.001549000 & 0.000037000 \\ 6 & 13.025738000 & 1.220291000 & 0.000024000 \\ 1 & 13.585235000 & 2.149458000 & 0.000029000 \\ 6 & 9.420478000 & -2.408094000 & 0.000001000 \\ 1 & 8.869341000 & -3.342269000 & 0.000002000 \\ 1 & -1.231313000 & 2.490460000 & -0.000026000 \\ 1 & -6.158148000 & 2.503629000 & -0.000020000 \\ 6 & -6.161653000 & -2.505331000 & -0.000011000 \\ 6 & -1.228521000 & -2.491722000 & -0.000019000 \\ 6 & 4.935742000 & 0.721360000 & -0.000015000 \\ 6 & 6.173658000 & 1.420218000 & -0.000012000 \\ 6 & -1.418420000 & -0.000012000\end{array}$




$\begin{array}{rrrr}1 & 6.158147000 & -2.503630000 & -0.000012000 \\ 1 & 3.697568000 & 2.486082000 & -0.000021000 \\ 1 & 3.696600000 & -2.483219000 & -0.000011000 \\ 6 & 1.228915000 & 1.406328000 & -0.000021000 \\ 6 & 1.227785000 & -1.405123000 & -0.000016000 \\ 1 & 1.228520000 & 2.491723000 & -0.000021000 \\ 1 & 1.231312000 & -2.490462000 & -0.000018000 \\ 6 & 2.476774000 & -0.723407000 & -0.000015000 \\ 6 & 2.476150000 & 0.726328000 & -0.000018000\end{array}$

$\begin{array}{lccc}\mathrm{Q} 2 & (n=3) & & \\ 6 & 8.584476000 & 0.728998000 & 0.000002000 \\ 6 & 6.158398000 & -0.721563000 & -0.000021000 \\ 6 & 6.158398000 & 0.721563000 & 0.000002000 \\ 6 & 12.157763000 & 0.000000000 & -0.000005000 \\ 6 & 10.764287000 & 0.000000000 & -0.000008000 \\ 6 & 8.584476000 & -0.728998000 & -0.000021000 \\ 6 & 9.979239000 & -1.175075000 & -0.000028000 \\ 6 & 4.926956000 & -1.399814000 & -0.000031000 \\ 1 & 4.927789000 & -2.485665000 & -0.000049000 \\ 6 & 7.400681000 & -1.421499000 & -0.000032000 \\ 6 & 12.038396000 & -2.439903000 & -0.000045000 \\ 1 & 12.545273000 & -3.399196000 & -0.000060000 \\ 6 & 12.834337000 & -1.252814000 & -0.000025000 \\ 6 & 7.400681000 & 1.421500000 & 0.000013000 \\ 6 & 12.834337000 & 1.252814000 & 0.000017000 \\ 6 & 14.247251000 & 1.221052000 & 0.000020000 \\ 1 & 14.807577000 & 2.149927000 & 0.000037000 \\ 6 & 10.644039000 & -2.409006000 & -0.000047000 \\ 1 & 10.092871000 & -3.343340000 & -0.000063000 \\ 6 & 9.979239000 & 1.175075000 & 0.000011000 \\ 6 & 12.038396000 & 2.439903000 & 0.000037000 \\ 1 & 12.545273000 & 3.399196000 & 0.000054000 \\ 6 & 14.924238000 & 0.000000000 & 0.000001000 \\ 1 & 16.008708000 & 0.000000000 & 0.000004000 \\ 6 & 14.247251000 & -1.221051000 & -0.000021000 \\ 1 & 14.807577000 & -2.149927000 & -0.000035000 \\ 6 & 10.644039000 & 2.409006000 & 0.000034000 \\ 1 & 10.092870000 & 3.343340000 & 0.000049000 \\ 6 & 3.704884000 & 0.723521000 & 0.000003000 \\ 6 & 3.704885000 & -0.723520000 & -0.000020000 \\ 6 & -12.157776000 & 0.000000000 & 0.000014000 \\ 6 & -10.764310000 & 0.000000000 & 0.000007000 \\ 6 & -9.979254000 & 1.175076000 & 0.000024000 \\ 6 & 4.926956000 & 1.399815000 & 0.000013000\end{array}$




\begin{tabular}{|c|c|c|c|}
\hline & 4.927789000 & 2.485665000 & 0.000030000 \\
\hline 6 & 2.457594000 & 1.405213000 & 0.000014000 \\
\hline 6 & -12.038416000 & 2.439895000 & 0.000055000 \\
\hline 1 & -12.545279000 & 3.399195000 & 0.000074000 \\
\hline 6 & -12.834351000 & 1.252819000 & 0.000038000 \\
\hline 6 & 2.457593000 & -1.405214000 & -0.000031000 \\
\hline 6 & -12.834351000 & -1.252818000 & -0.000001000 \\
\hline 6 & -14.247272000 & -1.221051000 & 0.000008000 \\
\hline 1 & -14.807598000 & -2.149927000 & -0.000003000 \\
\hline 6 & -10.644042000 & 2.408996000 & 0.000049000 \\
\hline 1 & -10.092887000 & 3.343337000 & 0.000063000 \\
\hline 6 & -9.979254000 & -1.175075000 & -0.000013000 \\
\hline 6 & -12.038417000 & -2.439894000 & -0.000022000 \\
\hline 1 & -12.545280000 & -3.399194000 & -0.000033000 \\
\hline 6 & -14.924258000 & 0.000000000 & 0.000030000 \\
\hline 1 & -16.008729000 & 0.000001000 & 0.000036000 \\
\hline 6 & -14.247272000 & 1.221051000 & 0.000046000 \\
\hline 1 & -14.807598000 & 2.149927000 & 0.000064000 \\
\hline 6 & -10.644042000 & -2.408995000 & -0.000027000 \\
\hline 1 & -10.092887000 & -3.343336000 & -0.000042000 \\
\hline 1 & 2.460581000 & 2.490903000 & 0.000031000 \\
\hline 1 & 7.387182000 & 2.506789000 & 0.000031000 \\
\hline 1 & 7.387182000 & -2.506789000 & -0.000050000 \\
\hline 1 & 2.460583000 & -2.490903000 & -0.000048000 \\
\hline 6 & -6.158439000 & 0.721542000 & 0.000011000 \\
\hline 6 & -6.158439000 & -0.721543000 & -0.000012000 \\
\hline 6 & -7.400650000 & 1.421483000 & 0.000024000 \\
\hline 1 & -7.387191000 & 2.506771000 & 0.000041000 \\
\hline 6 & -4.926907000 & 1.399814000 & 0.000020000 \\
\hline 6 & -4.926908000 & -1.399815000 & -0.000024000 \\
\hline 6 & -8.584485000 & -0.728953000 & -0.000009000 \\
\hline 6 & -8.584485000 & 0.728953000 & 0.000015000 \\
\hline 6 & -7.400651000 & -1.421483000 & -0.000022000 \\
\hline 1 & -7.387192000 & -2.506771000 & -0.000039000 \\
\hline 1 & -4.927798000 & 2.485665000 & 0.000037000 \\
\hline 1 & -4.927799000 & -2.485666000 & -0.000042000 \\
\hline 6 & -2.457585000 & 1.405216000 & 0.000018000 \\
\hline 6 & -2.457585000 & -1.405217000 & -0.000027000 \\
\hline 1 & -2.460553000 & 2.490908000 & 0.000035000 \\
\hline 1 & -2.460553000 & -2.490909000 & -0.000044000 \\
\hline 6 & -3.704892000 & -0.723550000 & -0.000015000 \\
\hline 6 & -3.704892000 & 0.723549000 & 0.000008000 \\
\hline 6 & -1.238731000 & -0.731393000 & -0.000017000 \\
\hline 6 & -1.238732000 & 0.731392000 & 0.000006000 \\
\hline 6 & -0.000017000 & -1.407545000 & -0.000029000 \\
\hline 6 & -0.000016000 & 1.407544000 & 0.000016000 \\
\hline
\end{tabular}




$\begin{array}{rrrr}1 & 0.000039000 & -2.493194000 & -0.000046000 \\ 1 & 0.000040000 & 2.493193000 & 0.000033000 \\ 6 & 1.238821000 & 0.731358000 & 0.000004000 \\ 6 & 1.238821000 & -0.731359000 & -0.000019000\end{array}$

$\mathrm{Q} 2(n=3) \beta \alpha \alpha \alpha$

$\begin{array}{cccc}6 & 8.582628000 & 0.730300000 & 0.000006000 \\ 6 & 6.157580000 & -0.723067000 & -0.000025000 \\ 6 & 6.156495000 & 0.722489000 & 0.000006000 \\ 6 & 12.149301000 & 0.000772000 & -0.000009000 \\ 6 & 10.756322000 & 0.000880000 & -0.000010000 \\ 6 & 8.583727000 & -0.729865000 & -0.000023000 \\ 6 & 9.968438000 & -1.174078000 & 0.000009000 \\ 6 & 4.924806000 & -1.400102000 & -0.000034000 \\ 1 & 4.923833000 & -2.486317000 & -0.000055000 \\ 6 & 7.393899000 & -1.420796000 & -0.000040000 \\ 6 & 12.027302000 & -2.441019000 & 0.000044000 \\ 1 & 12.535893000 & -3.399495000 & 0.000062000 \\ 6 & 12.826205000 & -1.251390000 & 0.000018000 \\ 6 & 7.394880000 & 1.421111000 & 0.000021000 \\ 6 & 12.825673000 & 1.252950000 & -0.000029000 \\ 6 & 14.236993000 & 1.221341000 & -0.000025000 \\ 1 & 14.796903000 & 2.150527000 & -0.000039000 \\ 6 & 10.637831000 & -2.412012000 & 0.000041000 \\ 1 & 10.086766000 & -3.346252000 & 0.000049000 \\ 6 & 9.970116000 & 1.175235000 & -0.000025000 \\ 6 & 12.028525000 & 2.442022000 & -0.000048000 \\ 1 & 12.536497000 & 3.400810000 & -0.000063000 \\ 6 & 14.914207000 & 0.001092000 & -0.000003000 \\ 1 & 15.998550000 & 0.000210000 & -0.000001000 \\ 6 & 14.235779000 & -1.220614000 & 0.000016000 \\ 1 & 14.796646000 & -2.149174000 & 0.000036000 \\ 6 & 10.637956000 & 2.412853000 & -0.000045000 \\ 1 & 10.087187000 & 3.347207000 & -0.000049000 \\ 6 & 3.700619000 & 0.724802000 & 0.000003000 \\ 6 & 3.701633000 & -0.726270000 & -0.000023000 \\ 6 & -12.151111000 & 0.000717000 & 0.000013000 \\ 6 & -10.757374000 & 0.000079000 & 0.000005000 \\ 6 & -9.972118000 & 1.175319000 & 0.000050000 \\ 6 & 4.926023000 & 1.399172000 & 0.000015000 \\ 1 & 4.923607000 & 2.485324000 & 0.000035000 \\ 6 & 2.461686000 & 1.405328000 & 0.000015000 \\ 6 & -12.030990000 & 2.441380000 & 0.000110000 \\ 1 & -12.538023000 & 3.400668000 & 0.000151000 \\ 6 & -12.827227000 & 1.253823000 & 0.000065000 \\ 6 & 2.460304000 & -1.407125000 & -0.000036000\end{array}$




\begin{tabular}{cccc}
6 & -12.828536000 & -1.251778000 & -0.000029000 \\
6 & -14.240576000 & -1.219659000 & -0.000021000 \\
1 & -14.801771000 & -2.147984000 & -0.000052000 \\
6 & -10.637466000 & 2.410143000 & 0.000106000 \\
1 & -10.086051000 & 3.344320000 & 0.000134000 \\
6 & -9.972432000 & -1.175343000 & -0.000032000 \\
6 & -12.032224000 & -2.439933000 & -0.000073000 \\
1 & -12.540243000 & -3.398678000 & -0.000108000 \\
6 & -14.917294000 & 0.002142000 & 0.000026000 \\
1 & -16.001788000 & 0.001972000 & 0.000031000 \\
6 & -14.240015000 & 1.222618000 & 0.000068000 \\
1 & -14.799418000 & 2.152098000 & 0.000108000 \\
6 & -10.639166000 & -2.409592000 & -0.000072000 \\
1 & -10.087988000 & -3.344010000 & -0.000105000 \\
1 & 2.461395000 & 2.491102000 & 0.000033000 \\
1 & 7.378895000 & 2.506653000 & 0.000024000 \\
1 & 7.379524000 & -2.506449000 & -0.000043000 \\
1 & 2.460713000 & -2.493001000 & -0.000055000 \\
6 & -6.151584000 & 0.722882000 & 0.000012000 \\
6 & -6.152046000 & -0.723376000 & -0.000015000 \\
6 & -7.396039000 & 1.421932000 & 0.000024000 \\
1 & -7.381579000 & 2.507396000 & 0.000053000 \\
6 & -4.924276000 & 1.399343000 & 0.000026000 \\
6 & -4.925053000 & -1.400444000 & -0.000027000 \\
6 & -8.579325000 & -0.731082000 & -0.000009000 \\
6 & -8.578739000 & 0.730533000 & 0.000016000 \\
6 & -7.396438000 & -1.422459000 & -0.000027000 \\
1 & -7.382513000 & -2.508008000 & -0.000053000 \\
1 & -4.921821000 & 2.485430000 & 0.000044000 \\
1 & -4.923328000 & -2.486562000 & -0.000049000 \\
6 & -2.454710000 & 1.403468000 & 0.000028000 \\
6 & -2.455814000 & -1.405652000 & -0.000028000 \\
1 & -2.453121000 & 2.489345000 & 0.000043000 \\
1 & -2.454962000 & -2.491549000 & -0.000051000 \\
6 & -3.699543000 & -0.724145000 & -0.000015000 \\
6 & -3.699182000 & 0.722850000 & 0.000013000 \\
6 & -1.238923000 & -0.731023000 & -0.000019000 \\
6 & -1.238810000 & 0.728481000 & 0.000009000 \\
6 & 0.004134000 & -1.409159000 & -0.000032000 \\
\hline & 0.005463000 & 1.406234000 & 0.000016000 \\
1 & 0.004659000 & -2.494884000 & -0.000055000 \\
6 & 1.233636000 & 0.730322000 & 0.000004000 \\
6 & -0.733081000 & -0.000023000
\end{tabular}




\begin{tabular}{|c|c|c|c|}
\hline \multicolumn{4}{|c|}{$\mathrm{Q} 2(n=3) \alpha \beta \alpha \alpha$} \\
\hline & 8.563237000 & 0.737092000 & 0.000076000 \\
\hline 6 & & -0.748044000 & -0.000106000 \\
\hline 6 & 6.135020000 & 0.708407000 & -0.000082000 \\
\hline 6 & 12.137363000 & 0.028205000 & \\
\hline 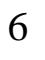 & $10.74278 ?$ & 0.0216 & 45000 \\
\hline 6 & 8.574058000 & -0.7332 & 0.000044000 \\
\hline 6 & 9.960706000 & -1.162037000 & 0.000181000 \\
\hline 6 & 4.928747000 & -1.430737000 & 4000 \\
\hline 1 & 4.9368540 & 56000 & -0.000 \\
\hline 6 & 7.395391000 & -1.4 & \\
\hline 5 & 12.027070000 & 000 & 33000 \\
\hline 1 & 12.540885000 & -3.3 & 9000 \\
\hline 6 & 12.820272000 & 6000 & 6000 \\
\hline O & 7.383131000 & & 7000 \\
\hline 6 & 12.807687000 & & \\
\hline & 14.2223240 & & \\
\hline 1 & 14.778584000 & 000 & 9000 \\
\hline U & 7844000 & 00 & 7000 \\
\hline 1 & 30000 & 000 & 000 \\
\hline & 9.95 & & \\
\hline & 12.0 & & \\
\hline & 12.510118000 & & 0000 \\
\hline & 14.9020390 & 00 & 000 \\
\hline 1 & 15.9 & & 000 \\
\hline 5 & & & \\
\hline & 14.7 & & 7000 \\
\hline & 10.6 & & 5000 \\
\hline 1 & 10.0 & 00 & 1000 \\
\hline 6 & & & \\
\hline & 2000 & -0.7 & 5000 \\
\hline 6 & 12.137156000 & 000 & 6000 \\
\hline 6 & -10.7 & 000 & 4000 \\
\hline 6 & -9.956810000 & & 0000 \\
\hline & & & 9000 \\
\hline & 4.916288000 & & -0.00 \\
\hline 6 & 2.459847000 & 1.37 & -0.000 \\
\hline 6 & -12.008768000 & 2.467270000 & 0.000520000 \\
\hline 1 & -12.511659000 & 3.428610000 & 0.000581000 \\
\hline 6 & -12.808076000 & 1.283284000 & 0.000436000 \\
\hline 6 & 2.462544000 & -1.444299000 & -0.000275000 \\
\hline 6 & -12.819599000 & -1.222292000 & 0.000208000 \\
\hline 6 & -14.227525000 & -1.186437000 & 0.00033500 \\
\hline & -14.791871000 & -2.112863000 & 0.000314000 \\
\hline & -10.615804000 & 2.429831000 & 0.000421000 \\
\hline & -10.059930000 & 3.361459000 & 0.000438000 \\
\hline
\end{tabular}




\begin{tabular}{|c|c|c|c|}
\hline 6 & -9.959586000 & -1.161744000 & -0.000006000 \\
\hline 6 & -12.025492000 & -2.417102000 & 0.000076000 \\
\hline 1 & -12.538997000 & -3.372952000 & 0.000111000 \\
\hline 6 & -14.901806000 & 0.040093000 & 0.000502000 \\
\hline 1 & -15.986365000 & 0.042729000 & 0.000616000 \\
\hline 6 & -14.222907000 & 1.256256000 & 0.000551000 \\
\hline 1 & -14.779676000 & 2.187243000 & 0.000652000 \\
\hline 6 & -10.636597000 & -2.395294000 & -0.000024000 \\
\hline 1 & -10.090646000 & -3.332679000 & -0.000100000 \\
\hline 1 & 2.459379000 & 2.456508000 & -0.000228000 \\
\hline 1 & 7.361643000 & 2.503842000 & 0.000006000 \\
\hline 1 & 7.392868000 & -2.520026000 & -0.000051000 \\
\hline 1 & 2.467652000 & -2.529586000 & -0.000323000 \\
\hline 6 & -6.135028000 & 0.710117000 & -0.000176000 \\
\hline 6 & -6.143817000 & -0.746948000 & -0.000223000 \\
\hline 6 & -7.383847000 & 1.419999000 & -0.000103000 \\
\hline 1 & -7.362404000 & 2.505238000 & -0.000023000 \\
\hline 6 & -4.921705000 & 1.379611000 & -0.000218000 \\
\hline 6 & -4.928585000 & -1.428709000 & -0.000356000 \\
\hline 6 & -8.573928000 & -0.732725000 & -0.000087000 \\
\hline 6 & -8.563362000 & 0.738117000 & -0.000010000 \\
\hline 6 & -7.394698000 & -1.433771000 & -0.000175000 \\
\hline 1 & -7.392238000 & -2.519099000 & -0.000251000 \\
\hline 1 & -4.917054000 & 2.465556000 & -0.000174000 \\
\hline 1 & -4.935916000 & -2.514496000 & -0.000397000 \\
\hline 6 & -2.459833000 & 1.373852000 & -0.000454000 \\
\hline 6 & -2.462463000 & -1.441630000 & -0.000514000 \\
\hline 1 & -2.459802000 & 2.459663000 & -0.000397000 \\
\hline 1 & -2.466838000 & -2.527177000 & -0.000537000 \\
\hline 6 & -3.687563000 & -0.759186000 & -0.000407000 \\
\hline 6 & -3.682280000 & 0.696351000 & -0.000377000 \\
\hline 6 & -1.229984000 & -0.766995000 & -0.000453000 \\
\hline 6 & -1.229191000 & 0.693996000 & -0.000389000 \\
\hline 6 & -0.001205000 & -1.445336000 & -0.000436000 \\
\hline 6 & -0.001592000 & 1.372475000 & -0.000311000 \\
\hline 1 & -0.000165000 & -2.530912000 & -0.000427000 \\
\hline 1 & 0.000701000 & 2.458177000 & -0.000343000 \\
\hline 6 & 1.227672000 & 0.694368000 & -0.000330000 \\
\hline 6 & 1.230396000 & -0.766926000 & -0.000308000 \\
\hline \multicolumn{4}{|c|}{$\mathrm{Q} 2(n=3) \beta \beta \alpha \alpha$} \\
\hline 6 & 8.565990000 & 0.732637000 & 0.000002000 \\
\hline 6 & 6.137863000 & -0.739834000 & -0.000014000 \\
\hline 6 & 6.136755000 & 0.718775000 & 0.000011000 \\
\hline 6 & 12.135613000 & 0.002396000 & -0.000029000 \\
\hline 6 & 10.740773000 & 0.003127000 & -0.000021000 \\
\hline
\end{tabular}




$\begin{array}{cccc}6 & 8.568412000 & -0.740365000 & -0.000026000 \\ 6 & 9.952748000 & -1.176074000 & 0.000011000 \\ 6 & 4.920889000 & -1.414471000 & -0.000017000 \\ 1 & 4.920464000 & -2.500259000 & -0.000035000 \\ 6 & 7.386388000 & -1.433880000 & -0.000032000 \\ 6 & 12.012281000 & -2.441781000 & 0.000045000 \\ 1 & 12.521405000 & -3.399930000 & 0.000069000 \\ 6 & 12.811945000 & -1.250755000 & 0.000006000 \\ 6 & 7.390308000 & 1.420910000 & 0.000019000 \\ 6 & 12.812577000 & 1.254743000 & -0.000062000 \\ 6 & 14.226773000 & 1.221265000 & -0.000066000 \\ 1 & 14.787771000 & 2.149718000 & -0.000090000 \\ 6 & 10.623485000 & -2.413003000 & 0.000049000 \\ 1 & 10.072529000 & -3.347475000 & 0.000064000 \\ 6 & 9.960599000 & 1.177421000 & -0.000044000 \\ 6 & 12.018671000 & 2.443191000 & -0.000087000 \\ 1 & 12.526728000 & 3.401801000 & -0.000113000 \\ 6 & 14.900148000 & 0.001571000 & -0.000038000 \\ 1 & 15.984708000 & -0.000928000 & -0.000044000 \\ 6 & 14.220368000 & -1.221462000 & -0.000005000 \\ 1 & 14.780509000 & -2.150438000 & 0.000021000 \\ 6 & 10.626128000 & 2.412623000 & -0.000077000 \\ 1 & 10.074477000 & 3.346729000 & -0.000084000 \\ 6 & 3.683130000 & 0.718588000 & 0.000021000 \\ 6 & 3.682023000 & -0.737261000 & -0.000005000 \\ 6 & -12.135489000 & -0.006727000 & -0.000007000 \\ 6 & -10.740589000 & -0.006721000 & -0.000004000 \\ 6 & -9.954187000 & 1.173416000 & 0.000058000 \\ 6 & 4.928268000 & 1.394900000 & 0.000024000 \\ 1 & 4.928923000 & 2.480767000 & 0.000042000 \\ 6 & 2.466163000 & 1.402503000 & 0.000036000 \\ 6 & -12.014445000 & 2.437514000 & 0.000112000 \\ 1 & -12.523795000 & 3.395556000 & 0.000157000 \\ 6 & -12.812150000 & 1.246110000 & 0.000050000 \\ 6 & 2.455635000 & -1.413273000 & -0.000012000 \\ 6 & -12.812017000 & -1.259421000 & -0.000068000 \\ 6 & -14.225569000 & -1.226959000 & -0.000073000 \\ 1 & -14.787058000 & -2.155036000 & -0.000117000 \\ 6 & -10.624937000 & 2.409731000 & 0.000118000 \\ 1 & -10.075102000 & 3.344773000 & 0.000155000 \\ 6 & -9.959326000 & -1.180102000 & -0.000045000 \\ 6 & -12.524186000 & -3.406093000 & -0.000168000 \\ 6 & -14.899990000 & -0.006879000 & -0.000021000 \\ 6 & -0.006209000 & -0.000028000 \\ 6 & -1.215713000 & 0.000038000\end{array}$




\begin{tabular}{|c|c|c|c|}
\hline & -14.781014000 & 2.145077000 & 0.000084000 \\
\hline 6 & -10.624303000 & -2.415555000 & -0.000103000 \\
\hline 1 & -10.071542000 & -3.349114000 & -0.000140000 \\
\hline 1 & 2.470771000 & 2.488101000 & 0.000054000 \\
\hline 1 & 7.374533000 & 2.506233000 & 0.000015000 \\
\hline 1 & 7.376364000 & -2.519214000 & -0.000029000 \\
\hline 1 & 2.452745000 & -2.498926000 & -0.000027000 \\
\hline 6 & -6.138143000 & 0.742569000 & 0.000032000 \\
\hline 6 & -6.136220000 & -0.715979000 & 0.000003000 \\
\hline 6 & -7.388424000 & 1.434789000 & 0.000038000 \\
\hline 1 & -7.379247000 & 2.520059000 & 0.000071000 \\
\hline 6 & -4.922222000 & 1.418502000 & 0.000053000 \\
\hline 6 & -4.927260000 & -1.390817000 & -0.000002000 \\
\hline 6 & -8.565375000 & -0.733705000 & -0.000003000 \\
\hline 6 & -8.568587000 & 0.739406000 & 0.000021000 \\
\hline 6 & -7.388843000 & -1.419995000 & -0.000018000 \\
\hline 1 & -7.371996000 & -2.505384000 & -0.000048000 \\
\hline 1 & -4.922366000 & 2.504248000 & 0.000072000 \\
\hline 1 & -4.927081000 & -2.476729000 & -0.000026000 \\
\hline 6 & -2.456171000 & 1.419118000 & 0.000059000 \\
\hline 6 & -2.465796000 & -1.396398000 & 0.000004000 \\
\hline 1 & -2.453542000 & 2.504706000 & 0.000079000 \\
\hline 1 & -2.469963000 & -2.482107000 & -0.000015000 \\
\hline 6 & -3.683158000 & -0.713009000 & 0.000014000 \\
\hline 6 & -3.682449000 & 0.742493000 & 0.000043000 \\
\hline 6 & -1.229845000 & -0.721598000 & 0.000013000 \\
\hline 6 & -1.227092000 & 0.737725000 & 0.000041000 \\
\hline 6 & -0.005661000 & -1.405353000 & 0.000002000 \\
\hline 6 & 0.005968000 & 1.411398000 & 0.000045000 \\
\hline 1 & -0.010483000 & -2.490953000 & -0.000023000 \\
\hline 1 & 0.010485000 & 2.497001000 & 0.000071000 \\
\hline 6 & 1.229510000 & 0.727810000 & 0.000029000 \\
\hline 6 & 1.226684000 & -0.731599000 & 0.000004000 \\
\hline \multicolumn{4}{|c|}{$\mathrm{Q} 2(n=3) \alpha \beta \beta \alpha$} \\
\hline 6 & 8.579088000 & 0.731004000 & 0.000003000 \\
\hline 6 & 6.153227000 & -0.723402000 & -0.000022000 \\
\hline 6 & 6.152488000 & 0.723315000 & 0.000006000 \\
\hline 6 & 12.146958000 & 0.000532000 & -0.000013000 \\
\hline 6 & 10.753620000 & 0.000292000 & -0.000012000 \\
\hline 6 & 8.579842000 & -0.731064000 & -0.000022000 \\
\hline 6 & 9.966887000 & -1.174872000 & 0.000021000 \\
\hline 6 & 4.923890000 & -1.400024000 & -0.000028000 \\
\hline 1 & 4.922670000 & -2.486232000 & -0.000046000 \\
\hline 6 & 7.392905000 & -1.421812000 & -0.000036000 \\
\hline 6 & 12.025944000 & -2.441085000 & 0.000070000 \\
\hline
\end{tabular}




$\begin{array}{cccc}1 & 12.534449000 & -3.399591000 & 0.000097000 \\ 6 & 12.824101000 & -1.251706000 & 0.000029000 \\ 6 & 7.393254000 & 1.421878000 & 0.000017000 \\ 6 & 12.823234000 & 1.253067000 & -0.000049000 \\ 6 & 14.234642000 & 1.221779000 & -0.000047000 \\ 1 & 14.794481000 & 2.151041000 & -0.000072000 \\ 6 & 10.635713000 & -2.411857000 & 0.000067000 \\ 1 & 10.084393000 & -3.346085000 & 0.000083000 \\ 6 & 9.967493000 & 1.175165000 & -0.000039000 \\ 6 & 12.025944000 & 2.441929000 & -0.000081000 \\ 1 & 12.533706000 & 3.400836000 & -0.000106000 \\ 6 & 14.912020000 & 0.001360000 & -0.000011000 \\ 1 & 15.996441000 & 0.000868000 & -0.000011000 \\ 6 & 14.234370000 & -1.220352000 & 0.000024000 \\ 1 & 14.795527000 & -2.148756000 & 0.000054000 \\ 6 & 10.635005000 & 2.412257000 & -0.000073000 \\ 1 & 10.083782000 & 3.346463000 & -0.000086000 \\ 6 & 3.698550000 & 0.724486000 & 0.000006000 \\ 6 & 3.699325000 & -0.725150000 & -0.000018000 \\ 6 & -12.146947000 & 0.000618000 & 0.000009000 \\ 6 & -10.753613000 & 0.000317000 & 0.000003000 \\ 6 & -9.967463000 & 1.175128000 & 0.000065000 \\ 6 & 4.924095000 & 1.399583000 & 0.000015000 \\ 1 & 4.921752000 & 2.485739000 & 0.000033000 \\ 6 & 2.457721000 & 1.404921000 & 0.000018000 \\ 6 & -12.025837000 & 2.442001000 & 0.000141000 \\ 1 & -12.533558000 & 3.400928000 & 0.000191000 \\ 6 & -12.823147000 & 1.253175000 & 0.000076000 \\ 6 & 2.457689000 & -1.406119000 & -0.000029000 \\ 6 & -12.824165000 & -1.251583000 & -0.000053000 \\ 6 & -14.234366000 & -1.220197000 & -0.000047000 \\ 1 & -14.795617000 & -2.148544000 & -0.000091000 \\ 6 & -10.634893000 & 2.412272000 & 0.000138000 \\ 1 & -10.083613000 & 3.346439000 & 0.000176000 \\ 6 & -9.966865000 & -1.174870000 & -0.000047000 \\ 6 & -12.025987000 & -2.441025000 & -0.000109000 \\ 1 & -12.534577000 & -3.399490000 & -0.000158000 \\ 6 & -14.912005000 & 0.001594000 & 0.000015000 \\ 1 & -15.996423000 & 0.001084000 & 0.000017000 \\ 6 & -14.234592000 & 1.221930000 & 0.000075000 \\ 1 & -14.794344000 & 2.151248000 & 0.000127000 \\ 6 & -10.635817000 & -2.411861000 & -0.000104000 \\ 1 & -10.084513000 & -3.346104000 & -0.000146000 \\ 1 & 2.456971000 & 2.490747000 & 0.000037000 \\ 6 & 7.378049000 & 2.507407000 & 0.000014000 \\ 6 & -2.507437000 & -0.000032000\end{array}$




$\begin{array}{rrrc}1 & 2.457751000 & -2.492049000 & -0.000045000 \\ 6 & -6.152460000 & 0.723119000 & 0.000015000 \\ 6 & -6.153300000 & -0.723599000 & -0.000012000 \\ 6 & -7.393194000 & 1.421709000 & 0.000026000 \\ 1 & -7.377897000 & 2.507237000 & 0.000062000 \\ 6 & -4.924061000 & 1.399354000 & 0.000033000 \\ 6 & -4.923886000 & -1.400281000 & -0.000023000 \\ 6 & -8.579919000 & -0.731153000 & -0.000011000 \\ 6 & -8.579042000 & 0.730897000 & 0.000018000 \\ 6 & -7.392911000 & -1.421917000 & -0.000028000 \\ 1 & -7.378687000 & -2.507551000 & -0.000060000 \\ 1 & -4.921630000 & 2.485510000 & 0.000053000 \\ 1 & -4.922669000 & -2.486494000 & -0.000046000 \\ 6 & -2.457930000 & 1.404503000 & 0.000033000 \\ 6 & -2.457784000 & -1.406464000 & -0.000022000 \\ 1 & -2.456955000 & 2.490385000 & 0.000053000 \\ 1 & -2.457767000 & -2.492371000 & -0.000044000 \\ 6 & -3.699408000 & -0.725413000 & -0.000010000 \\ 6 & -3.698625000 & 0.724185000 & 0.000019000 \\ 6 & -1.236096000 & -0.732282000 & -0.000013000 \\ 6 & -1.235658000 & 0.730000000 & 0.000015000 \\ 6 & 0.000035000 & -1.409228000 & -0.000025000 \\ 6 & -0.000017000 & 1.406671000 & 0.000023000 \\ 1 & 0.000079000 & -2.494965000 & -0.000046000 \\ 1 & -0.000180000 & 2.492441000 & 0.000045000 \\ 6 & 1.235798000 & 0.730014000 & 0.000009000 \\ 6 & 1.236106000 & -0.732188000 & -0.000016000\end{array}$

$\begin{array}{lrrr}\mathrm{Q} 2(n=4) & & \\ 6 & -9.812913000 & -0.730478000 & -0.001070000 \\ 6 & 4.930991000 & -0.723165000 & 0.000383000 \\ 6 & 4.931031000 & 0.723392000 & 0.000383000 \\ 6 & -9.812737000 & 0.729768000 & -0.001070000 \\ 6 & 6.159850000 & -1.401261000 & 0.000473000 \\ 1 & 6.158591000 & -2.487077000 & 0.000453000 \\ 6 & 3.689233000 & -1.405097000 & 0.000284000 \\ 6 & 3.689323000 & 1.405392000 & 0.000285000 \\ 6 & 9.811352000 & -0.732636000 & 0.000751000 \\ 6 & 7.382940000 & 0.724356000 & 0.000564000 \\ 6 & 7.382894000 & -0.724276000 & 0.000565000 \\ 6 & 9.811404000 & 0.732577000 & 0.000755000 \\ 6 & 6.159938000 & 1.401416000 & 0.000469000 \\ 1 & 6.158745000 & 2.487232000 & 0.000449000 \\ 6 & 8.630801000 & 1.424174000 & 0.000665000 \\ 6 & 8.630713000 & -1.424168000 & 0.000661000\end{array}$




\begin{tabular}{|c|c|c|c|}
\hline & 8.615729000 & 2.509423000 & \\
\hline & 3.691612000 & -2.490748000 & 0.000260000 \\
\hline & 691757000 & 2.491044000 & .000261000 \\
\hline & 8.615586000 & -2.509416000 & 0.000652000 \\
\hline & -7.384378000 & 0.722225000 & -0.000784000 \\
\hline & -7.384588000 & -0.722349000 & -0.000785000 \\
\hline & -6.156147000 & 1.401007000 & 0000 \\
\hline & -6.155921000 & -1.400511000 & 1000 \\
\hline & -8.628887000 & -1.422994000 & -0.000942000 \\
\hline & -8.628648000 & 1.422070000 & 7000 \\
\hline & 10000 & $-2.50 \varepsilon$ & 9000 \\
\hline & -6.1 & $2.4 \varepsilon$ & \\
\hline & 70000 & -2.486 & -0.000 \\
\hline & -8.615272000 & $2.50^{\prime}$ & 0000 \\
\hline & 0.003644000 & 0.73 & -0.000 \\
\hline & 0.00 & -0.7 & \\
\hline & 2.47 & -0.7 & 000 \\
\hline & 2.474 & & \\
\hline & 1.226879000 & -1.407 & 3000 \\
\hline & 1.226 & 1.40 & \\
\hline & 1.23 & -2.49 & 000 \\
\hline & 1.23 & 2.45 & \\
\hline & -1.227892000 & -1.407 & -0.00 \\
\hline & 00000 & 1.40 & -0.0 \\
\hline & -1.22 & -2.49 & -0. \\
\hline & -1.22 & 2.49 & \\
\hline & -4.932502000 & & 7000 \\
\hline & -4.9 & -0.72 & -0 . \\
\hline & -2.4 & -0.7 & -0. \\
\hline & -2.4 & 0.73 & -0 . \\
\hline & -3.686980000 & -1.40 & -0.0 \\
\hline & -3.68 & 1.40 & \\
\hline & -3.6 & $-2.4 C$ & -0.0 \\
\hline & -3.69 & 2.49 & -0.0 \\
\hline & 8000 & -0.0 & 9900 \\
\hline & 11.989640000 & -0.00( & 2000 \\
\hline & 11.205507000 & -1.176 & 300 \\
\hline & 13.264769000 & -2.44 & 3000 \\
\hline & 13.772528000 & -3.400298000 & 7300 \\
\hline & 14.061000000 & -1.253203000 & 0.00146700 \\
\hline & 14.061086000 & 1.252851000 & 0.00144800 \\
\hline & 15.473905000 & 1.221191000 & 0.00174700 \\
\hline & 16.034292000 & 2.149986000 & 86000 \\
\hline & 11.871267000 & -2.410926000 & 0.00110100 \\
\hline & 11.319760000 & -3.345035000 & 0.00103300 \\
\hline & 11.205582000 & 1.175913000 & 0.00091700 \\
\hline
\end{tabular}




\begin{tabular}{|c|c|c|c|}
\hline 6 & 13.264928000 & 2.441219000 & 0.001313000 \\
\hline 1 & 13.772756000 & 3.399966000 & 0.001419000 \\
\hline 6 & 16.150804000 & -0.000248000 & 0.001891000 \\
\hline 1 & 17.235302000 & -0.000278000 & 0.002106000 \\
\hline 6 & 15.473829000 & -1.221635000 & 0.001764000 \\
\hline 1 & 16.034147000 & -2.150472000 & 0.001893000 \\
\hline 6 & 11.871429000 & 2.410724000 & 0.001063000 \\
\hline 1 & 11.319980000 & 3.344867000 & 0.000985000 \\
\hline 6 & -13.385765000 & -0.000079000 & -0.001191000 \\
\hline 6 & -11.991597000 & -0.000135000 & -0.001236000 \\
\hline 6 & -11.207140000 & -1.175870000 & -0.001176000 \\
\hline 6 & -13.266457000 & -2.441153000 & -0.001112000 \\
\hline 1 & -13.773976000 & -3.400073000 & -0.001078000 \\
\hline 6 & -14.062475000 & -1.253182000 & -0.001140000 \\
\hline 6 & -14.062374000 & 1.253078000 & -0.001136000 \\
\hline 6 & -15.475421000 & 1.221367000 & -0.001074000 \\
\hline 1 & -16.035749000 & 2.150213000 & -0.001041000 \\
\hline 6 & -11.872357000 & -2.410541000 & -0.001115000 \\
\hline 1 & -11.321056000 & -3.344779000 & -0.001073000 \\
\hline 6 & -11.207088000 & 1.175467000 & -0.001170000 \\
\hline 6 & -13.266274000 & 2.440974000 & -0.001103000 \\
\hline 1 & -13.773693000 & 3.399947000 & -0.001067000 \\
\hline 6 & -16.152520000 & 0.000029000 & -0.001055000 \\
\hline 1 & -17.236979000 & 0.000057000 & -0.001023000 \\
\hline 6 & -15.475496000 & -1.221374000 & -0.001078000 \\
\hline 1 & -16.035902000 & -2.150172000 & -0.001048000 \\
\hline 6 & -11.872154000 & 2.410199000 & -0.001106000 \\
\hline 1 & -11.320771000 & 3.344393000 & -0.001062000 \\
\hline \multicolumn{4}{|c|}{$\mathrm{Q} 2(n=4) \beta \alpha \alpha \alpha$} \\
\hline 6 & 9.808056000 & 0.731080000 & -0.000963000 \\
\hline 6 & -4.926804000 & 0.723565000 & 0.000255000 \\
\hline 6 & -4.926803000 & -0.723564000 & 0.000252000 \\
\hline 6 & 9.808075000 & -0.731149000 & -0.000967000 \\
\hline 6 & -6.155681000 & 1.401107000 & 0.000395000 \\
\hline 1 & -6.155345000 & 2.486984000 & 0.000397000 \\
\hline 6 & -3.686130000 & 1.404883000 & 0.000124000 \\
\hline 6 & -3.686135000 & -1.404881000 & 0.000118000 \\
\hline 6 & -9.806282000 & 0.732280000 & 0.000842000 \\
\hline 6 & -7.378446000 & -0.724345000 & 0.000533000 \\
\hline 6 & -7.378446000 & 0.724347000 & 0.000536000 \\
\hline 6 & -9.806285000 & -0.732281000 & 0.000838000 \\
\hline 6 & -6.155684000 & -1.401106000 & 0.000390000 \\
\hline 1 & -6.155347000 & -2.486983000 & 0.000388000 \\
\hline 6 & -8.625654000 & -1.423810000 & 0.000685000 \\
\hline 6 & -8.625653000 & 1.423811000 & 0.000692000 \\
\hline
\end{tabular}




$\begin{array}{rrrr}1 & -8.611791000 & -2.509145000 & 0.000684000 \\ 1 & -3.688143000 & 2.490589000 & 0.000128000 \\ 1 & -3.688143000 & -2.490587000 & 0.000118000 \\ 1 & -8.611795000 & 2.509146000 & 0.000690000 \\ 6 & 7.382523000 & -0.723344000 & -0.000819000 \\ 6 & 7.382530000 & 0.723334000 & -0.000815000 \\ 6 & 6.155485000 & -1.400945000 & -0.000737000 \\ 6 & 6.155442000 & 1.400902000 & -0.000730000 \\ 6 & 8.624264000 & 1.423168000 & -0.000890000 \\ 6 & 8.624328000 & -1.423176000 & -0.000898000 \\ 1 & 8.610721000 & 2.508501000 & -0.000891000 \\ 1 & 6.157455000 & -2.486864000 & -0.000740000 \\ 1 & 6.157292000 & 2.486832000 & -0.000727000 \\ 1 & 8.610644000 & -2.508535000 & -0.000906000 \\ 6 & -0.001690000 & -0.733505000 & -0.000243000 \\ 6 & -0.001695000 & 0.733507000 & -0.000240000 \\ 6 & -2.470579000 & 0.728929000 & -0.000003000 \\ 6 & -2.470575000 & -0.728925000 & -0.000006000 \\ 6 & -1.224112000 & 1.407517000 & -0.000124000 \\ 6 & -1.224123000 & -1.407514000 & -0.000129000 \\ 1 & -1.226204000 & 2.493188000 & -0.000122000 \\ 1 & -1.226206000 & -2.493185000 & -0.000131000 \\ 6 & 1.230865000 & 1.408081000 & -0.000348000 \\ 6 & 1.230849000 & -1.408087000 & -0.000354000 \\ 6 & -1.232172000 & 2.493790000 & -0.000348000 \\ 6 & -11.314223000 & 3.344576000 & 0.001096000 \\ 6 & 1.232160000 & -2.493796000 & -0.000357000 \\ 6 & 4.930699000 & -0.724583000 & -0.000648000 \\ 6 & 4.930720000 & 0.724490000 & -0.000644000 \\ 6 & 2.470364000 & 0.731207000 & -0.000454000 \\ 6 & 2.470361000 & -0.731227000 & -0.000457000 \\ 6 & 3.688785000 & 1.405503000 & -0.000549000 \\ 6 & 3.688767000 & -1.405546000 & -0.000555000 \\ 6 & -1.692499000 & 2.491280000 & -0.000546000 \\ 6 & -11.692452000 & -2.491328000 & -0.000557000 \\ 6 & -13.2578585000 & -1.175723000 & 0.001051000\end{array}$




\begin{tabular}{|c|c|c|c|}
\hline 6 & -13.258588000 & -2.440825000 & 0.001451000 \\
\hline 1 & -13.766148000 & -3.399765000 & 0.001562000 \\
\hline 6 & -16.143876000 & 0.000010000 & 0.001988000 \\
\hline 1 & -17.228399000 & 0.000008000 & 0.002204000 \\
\hline 6 & -15.467110000 & 1.221172000 & 0.001851000 \\
\hline 1 & -16.027490000 & 2.150016000 & 0.001966000 \\
\hline 6 & -11.865550000 & -2.410314000 & 0.001198000 \\
\hline 1 & -11.314229000 & -3.344570000 & 0.001118000 \\
\hline 6 & 13.375745000 & 0.000015000 & -0.001048000 \\
\hline 6 & 11.982260000 & -0.000021000 & -0.001065000 \\
\hline 6 & 11.197720000 & 1.175965000 & -0.001022000 \\
\hline 6 & 13.255773000 & 2.441590000 & -0.000990000 \\
\hline 1 & 13.763254000 & 3.400530000 & -0.000964000 \\
\hline 6 & 14.052037000 & 1.252846000 & -0.001016000 \\
\hline 6 & 14.052083000 & -1.252788000 & -0.001028000 \\
\hline 6 & 15.463648000 & -1.221061000 & -0.000998000 \\
\hline 1 & 16.024043000 & -2.149874000 & -0.000982000 \\
\hline 6 & 11.863966000 & 2.411965000 & -0.000986000 \\
\hline 1 & 11.313362000 & 3.346567000 & -0.000953000 \\
\hline 6 & 11.197789000 & -1.176024000 & -0.001032000 \\
\hline 6 & 13.255876000 & -2.441561000 & -0.001014000 \\
\hline 1 & 13.763389000 & -3.400483000 & -0.000997000 \\
\hline 6 & 16.140518000 & 0.000071000 & -0.000982000 \\
\hline 1 & 17.224963000 & 0.000099000 & -0.000959000 \\
\hline 6 & 15.463590000 & 1.221185000 & -0.000986000 \\
\hline 1 & 16.023953000 & 2.150018000 & -0.000961000 \\
\hline 6 & 11.864058000 & -2.411995000 & -0.001009000 \\
\hline 1 & 11.313490000 & -3.346617000 & -0.000984000 \\
\hline \multicolumn{4}{|c|}{$\mathrm{Q} 2(n=4) \alpha \beta \alpha \alpha$} \\
\hline 6 & 9.795126000 & 0.740062000 & -0.000891000 \\
\hline 6 & -4.914757000 & 0.693068000 & 0.000451000 \\
\hline 6 & -4.921688000 & -0.760136000 & 0.000447000 \\
\hline 6 & 9.807487000 & -0.728753000 & -0.000896000 \\
\hline 6 & -6.150392000 & 1.377776000 & 0.000557000 \\
\hline 1 & -6.145254000 & 2.463642000 & 0.000560000 \\
\hline 6 & -3.688074000 & 1.370244000 & 0.000349000 \\
\hline 6 & -3.692475000 & -1.443869000 & 0.000341000 \\
\hline 6 & -9.795406000 & 0.740312000 & 0.000872000 \\
\hline 6 & -7.377798000 & -0.745608000 & 0.000656000 \\
\hline 6 & -7.367527000 & 0.708790000 & 0.000659000 \\
\hline 6 & -9.807481000 & -0.728623000 & 0.000871000 \\
\hline 6 & -6.159664000 & -1.429059000 & 0.000549000 \\
\hline 1 & -6.167653000 & -2.514760000 & 0.000547000 \\
\hline 6 & -8.627957000 & -1.431040000 & 0.000763000 \\
\hline 6 & -8.613870000 & 1.420612000 & 0.000768000 \\
\hline
\end{tabular}




\begin{tabular}{|c|c|c|c|}
\hline & -8.625873000 & -2.516356000 & \\
\hline & -3.688240000 & 2.455927000 & 0.000351000 \\
\hline & -3.698201000 & -2.529390000 & 0.000338000 \\
\hline & -8.590679000 & 2.505843000 & 0.000773000 \\
\hline & 7.377898000 & -0.746085000 & -0.000654000 \\
\hline & 7.367447000 & 0.708485000 & \\
\hline & 6.159736 & -1.429 & 000 \\
\hline & 6.150 & 1.3773 & \\
\hline & 8.613805000 & 1.420361000 & -0.000772000 \\
\hline & 8.62 & -1.43 & -0.0 \\
\hline & 8.59 & 2.50 & 000 \\
\hline & 6.16 & -2.5 & 10000 \\
\hline & 526000 & 2.46316 & 6000 \\
\hline & 8.626 & -2.516 & 000 \\
\hline & 0.000120000 & -0.77 & 0.00 \\
\hline & 0.00 & 0.6 & \\
\hline & -2.4 & 0.6 & 00 \\
\hline & -2.46 & -0.7 & 000 \\
\hline & -1.229125000 & 1.36 & 1000 \\
\hline & -1.23 & -1.4 & 000 \\
\hline & -1.22 & 2.452 & 000 \\
\hline & -1.23 & -2.5 & 9000 \\
\hline & 1.229 & 1.366 & -0.00 \\
\hline & 1.23 & -1.4 & -0. \\
\hline & 1.22 & 2.45 & -0. \\
\hline & 1.23 & -2.53 & -0 . \\
\hline & 4.921 & -0.76 & -0.0 \\
\hline & 4.91 & 0.65 & -0.0 \\
\hline & 2.45 & 0.6 & -0. \\
\hline & 2.46 & -0.77 & -0. \\
\hline & 3.688 & 1.369 & -0.00 \\
\hline & 3.69 & -1.4 & -0.0 \\
\hline & 3.68 & 2.45 & -0.0 \\
\hline & 3.69 & -2.52 & -0.00 \\
\hline & 8000 & & \\
\hline & 3000 & $0.02^{\prime}$ & 07100 \\
\hline & -11.18 & 1.196 & 1100 \\
\hline & -13.23 & 8000 & 0.0 \\
\hline & -13.741130000 & 3.435872000 & 0.00130000 \\
\hline & -14.040030000 & 1.291141000 & 0.00129900 \\
\hline & -14.053764000 & -1.214325000 & 0.00127100 \\
\hline & -15.461837000 & -1.177102000 & 0.00141800 \\
\hline & -16.026728000 & -2.103271000 & 4680 \\
\hline & -11.846170000 & 2.434947000 & 0.00109700 \\
\hline & -11.289054000 & 3.365846000 & 0.00105400 \\
\hline & -11.193892000 & -1.156330000 & 0.0009870 \\
\hline
\end{tabular}




\begin{tabular}{|c|c|c|c|}
\hline 6 & -13.261048000 & -2.409712000 & 0.001181000 \\
\hline 1 & -13.775236000 & -3.365204000 & 0.001227000 \\
\hline 6 & -16.135034000 & 0.049792000 & 0.001500000 \\
\hline 1 & -17.219581000 & 0.053719000 & 0.001611000 \\
\hline 6 & -15.454879000 & 1.265392000 & 0.001445000 \\
\hline 1 & -16.011013000 & 2.196746000 & 0.001514000 \\
\hline 6 & -11.871728000 & -2.389200000 & 0.001045000 \\
\hline 1 & -11.326905000 & -3.327176000 & 0.000987000 \\
\hline 6 & 13.370213000 & 0.035144000 & -0.001259000 \\
\hline 6 & 11.975813000 & 0.028008000 & -0.001123000 \\
\hline 6 & 11.188621000 & 1.196422000 & -0.001038000 \\
\hline 6 & 13.239133000 & 2.474088000 & -0.001236000 \\
\hline 1 & 13.740806000 & 3.436042000 & -0.001282000 \\
\hline 6 & 14.039898000 & 1.291370000 & -0.001321000 \\
\hline 6 & 14.053822000 & -1.214116000 & -0.001324000 \\
\hline 6 & 15.461779000 & -1.176882000 & -0.001460000 \\
\hline 1 & 16.026719000 & -2.103023000 & -0.001514000 \\
\hline 6 & 11.845965000 & 2.434947000 & -0.001096000 \\
\hline 1 & 11.288927000 & 3.365899000 & -0.001035000 \\
\hline 6 & 11.193703000 & -1.156349000 & -0.001040000 \\
\hline 6 & 13.261064000 & -2.409527000 & -0.001242000 \\
\hline 1 & 13.775245000 & -3.365027000 & -0.001292000 \\
\hline 6 & 16.134973000 & 0.050122000 & -0.001526000 \\
\hline 1 & 17.219526000 & 0.054038000 & -0.001631000 \\
\hline 6 & 15.454847000 & 1.265616000 & -0.001458000 \\
\hline 1 & 16.010925000 & 2.197000000 & -0.001511000 \\
\hline 6 & 11.871804000 & -2.389120000 & -0.001103000 \\
\hline 1 & 11.327184000 & -3.327213000 & -0.001046000 \\
\hline \multicolumn{4}{|c|}{$\mathrm{Q} 2(n=4) \beta \beta \alpha \alpha$} \\
\hline 6 & 9.803771000 & 0.738664000 & -0.000894000 \\
\hline 6 & -4.918179000 & 0.708614000 & 0.000451000 \\
\hline 6 & -4.917923000 & -0.744654000 & 0.000451000 \\
\hline 6 & 9.798749000 & -0.730315000 & -0.000892000 \\
\hline 6 & -6.157767000 & 1.386713000 & 0.000556000 \\
\hline 1 & -6.158481000 & 2.472578000 & 0.000557000 \\
\hline 6 & -3.695637000 & 1.392537000 & 0.000348000 \\
\hline 6 & -3.684827000 & -1.421605000 & 0.000346000 \\
\hline 6 & -9.799070000 & 0.730275000 & 0.000872000 \\
\hline 6 & -7.373968000 & -0.743262000 & 0.000659000 \\
\hline 6 & -7.371061000 & 0.711252000 & 0.000659000 \\
\hline 6 & -9.803725000 & -0.738803000 & 0.000873000 \\
\hline 6 & -6.152204000 & -1.420196000 & 0.000554000 \\
\hline 1 & -6.154384000 & -2.505932000 & 0.000553000 \\
\hline 6 & -8.620608000 & -1.435120000 & 0.000768000 \\
\hline 6 & -8.621235000 & 1.416676000 & 0.000766000 \\
\hline
\end{tabular}




\begin{tabular}{|c|c|c|c|}
\hline & -8.612947000 & -2.520411000 & \\
\hline & -3.701958000 & 2.478178000 & 0.000349000 \\
\hline & -3.684419000 & -2.507162000 & 0.000345000 \\
\hline & -8.603667000 & 2.502004000 & 0.000769000 \\
\hline & 7.370941000 & -0.710981000 & -0.000649000 \\
\hline & 7.374095000 & 0.743722000 & 1000 \\
\hline & 6.157852000 & -1.386141000 & 30000 \\
\hline & 6.152416000 & 1.420698000 & -0.00 \\
\hline & 8.62 & 1.435 & \\
\hline & 8.621 & -1.41 & 000 \\
\hline & 8.61 & & \\
\hline & 6.15 & -2.471972000 & -0.0 \\
\hline & 6.15 & 2.506 & -0.0 \\
\hline & 8.60 & -2.50 & -0.0 \\
\hline & 0.00 & -0.7 & \\
\hline & -0.00 & & 0.00 \\
\hline & -2.46 & 0.7 & 000 \\
\hline & -2.459782000 & -0.74 & 0.00 \\
\hline & -1.23 & 1.40 & 0.0 \\
\hline & -1.22 & -1.413 & 0.00 \\
\hline & -1.24 & 2.48 & \\
\hline & -1.21 & -2.49 & 0.00 \\
\hline & 1.222 & 1.41 & -0.0 \\
\hline & 1.23 & -1.40 & -0.00 \\
\hline & 1.21 & 2.49 & -0.0 \\
\hline & 1.24 & -2.4 & \\
\hline & 4.91 & -0.70 & -0.0 \\
\hline & 4.91 & 0.7 & -0.00 \\
\hline & 2.45 & 0.74 & -0.0 \\
\hline & 2.46 & -0.717 & -0.00 \\
\hline & 3.68 & 1.422 & -0.00 \\
\hline & 3.69 & -1.35 & -0.00 \\
\hline & 3.68 & 2.507 & -0.0 \\
\hline & 3.702210000 & -2.477456000 & -0.000 \\
\hline & 0366000 & & 1100 \\
\hline & 000 & 0.00 & 0.0 \\
\hline & $-11.1 \mathrm{C}$ & 1000 & 0.00 \\
\hline & -13.25 & 52000 & \\
\hline & -13.7 & 96000 & \\
\hline & -14.046 & 6000 & 0.00129300 \\
\hline & -14.047573000 & -1.245725000 & 0.00127200 \\
\hline & -15.455809000 & 546000 & 0.001 \\
\hline & -16.016055000 & -2.144530000 & 0.0014690 \\
\hline & -11.858326000 & 2.414529000 & 0.00109000 \\
\hline & -11.305851000 & 3.348192000 & 0.00104600 \\
\hline & -11.187981000 & -1.173452000 & 0.0009900 \\
\hline
\end{tabular}




$\begin{array}{lrrr}6 & -13.248873000 & -2.437128000 & 0.001185000 \\ 1 & -13.758272000 & -3.395182000 & 0.001233000 \\ 6 & -16.135166000 & 0.007981000 & 0.001496000 \\ 1 & -17.219720000 & 0.006448000 & 0.001606000 \\ 6 & -15.461161000 & 1.226967000 & 0.001438000 \\ 1 & -16.021957000 & 2.155519000 & 0.001504000 \\ 6 & -11.859646000 & -2.409675000 & 0.001050000 \\ 1 & -11.310151000 & -3.344926000 & 0.000995000 \\ 6 & 13.370297000 & -0.007587000 & -0.001261000 \\ 6 & 11.975875000 & -0.007336000 & -0.001123000 \\ 6 & 11.187854000 & 1.173168000 & -0.001042000 \\ 6 & 13.249012000 & 2.436540000 & -0.001250000 \\ 1 & 13.758457000 & 3.394574000 & -0.001302000 \\ 6 & 14.047698000 & 1.245064000 & -0.001330000 \\ 6 & 14.046204000 & -1.260489000 & -0.001320000 \\ 6 & 15.461058000 & -1.227724000 & -0.001459000 \\ 1 & 16.021744000 & -2.156341000 & -0.001510000 \\ 6 & 11.859852000 & 2.409266000 & -0.001109000 \\ 1 & 11.310606000 & 3.344664000 & -0.001053000 \\ 6 & 11.194591000 & -1.179659000 & -0.001035000 \\ 6 & 13.251370000 & -2.447145000 & -0.001231000 \\ 1 & 13.757788000 & -3.406605000 & -0.001275000 \\ 6 & 16.135098000 & -0.008887000 & -0.001530000 \\ 1 & 17.219659000 & -0.007402000 & -0.001636000 \\ 6 & 15.455809000 & 1.214794000 & -0.001467000 \\ 1 & 16.016158000 & 2.143718000 & -0.001524000 \\ 6 & 11.857990000 & -2.414876000 & -0.001090000 \\ 1 & 11.305548000 & -3.348562000 & -0.001028000\end{array}$

$\begin{array}{lrrr}\mathrm{Q} 2 & (n=4) \alpha \beta \beta \alpha \\ 6 & 9.805445000 & 0.731327000 & -0.000962000 \\ 6 & -4.926715000 & 0.723935000 & 0.000255000 \\ 6 & -4.926714000 & -0.723929000 & 0.000252000 \\ 6 & 9.805464000 & -0.731395000 & -0.000967000 \\ 6 & -6.154976000 & 1.401154000 & 0.000394000 \\ 1 & -6.154503000 & 2.487078000 & 0.000397000 \\ 6 & -3.687178000 & 1.405257000 & 0.000125000 \\ 6 & -3.687186000 & -1.405253000 & 0.000119000 \\ 6 & -9.805042000 & 0.732723000 & 0.000839000 \\ 6 & -7.378009000 & -0.724560000 & 0.000532000 \\ 6 & -7.378007000 & 0.724568000 & 0.000535000 \\ 6 & -9.805045000 & -0.732721000 & 0.000836000 \\ 6 & -6.154980000 & -1.401148000 & 0.000389000 \\ 1 & -6.154508000 & -2.487072000 & 0.000387000 \\ 6 & -8.623197000 & -1.424043000 & 0.000683000 \\ 6 & -8.623195000 & 1.424048000 & 0.000690000\end{array}$




\begin{tabular}{|c|c|c|c|}
\hline & -8.608583000 & -2.509404000 & \\
\hline & -3.689768000 & 2.490992000 & 0.000128000 \\
\hline & -3.689772000 & -2.490989000 & 0.000118000 \\
\hline & -8.608588000 & 2.509409000 & 0.000688000 \\
\hline & 7.379650000 & -0.723614000 & -0.000818000 \\
\hline & 7.379 & 0.723 & 4000 \\
\hline & 6.153540000 & -1.401152000 & 35000 \\
\hline & 6.153496000 & 1.401104000 & -0.000728000 \\
\hline 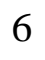 & 8.622 & 1.4 & 9000 \\
\hline & 8.622273000 & -1.4 & 7000 \\
\hline & 8.60 & & \\
\hline & 6.1 & -2.4 & 8000 \\
\hline & 6.15 & 2.4 & 5000 \\
\hline 1 & 900000 & -2.5 & 06000 \\
\hline 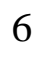 & 12000 & -0.73 & -0.0 \\
\hline & -0.0 & & \\
\hline & -2.4 & 0.7 & 2000 \\
\hline & -2.4 & -0.7 & -0.0 \\
\hline$U$ & -1.2 & 1.4 & -0.0 \\
\hline & -1.2 & -1.4 & -0.0 \\
\hline & -1.2 & & \\
\hline & -1.2 & -2.4 & \\
\hline & 1.22 & 1.4 & -0.0 \\
\hline & 1.2 & -1.4 & 000 \\
\hline & 1.2 & 2.4 & 000 \\
\hline & & & \\
\hline & 4.92 & -0.7 & 6000 \\
\hline & 4.9 & 0.7 & -0 . \\
\hline 0 & 2.4 & 0.7 & 2000 \\
\hline & & -0.7 & \\
\hline & & & \\
\hline & & -1.4 & -0. \\
\hline & 3.6 & 2.4 & 4000 \\
\hline . & & -2.4912 & 55000 \\
\hline & & & 3000 \\
\hline & & & 2000 \\
\hline 6 & -11.1 & 89000 & 0.00 \\
\hline 6 & -13.25 & & 30000 \\
\hline & & & 3600 \\
\hline & -14.045 & 1.252817000 & 0.00156800 \\
\hline & -14.049918000 & -1.252805000 & 0.00157800 \\
\hline 6 & -15.461541000 & -1.221184000 & 0.00186100 \\
\hline 1 & -16.021933000 & -2.150006000 & 0.00198400 \\
\hline & -11.861879000 & 2.411936000 & 0.001177000 \\
\hline & -11.310960000 & 3.346389000 & 0.00109300 \\
\hline & -11.195348000 & -1.176083000 & 0.00104900 \\
\hline
\end{tabular}




$\begin{array}{rrrr}6 & -13.253613000 & -2.441709000 & 0.001450000 \\ 1 & -13.761352000 & -3.400531000 & 0.001561000 \\ 6 & -16.138323000 & 0.000009000 & 0.001990000 \\ 1 & -17.222816000 & 0.000008000 & 0.002207000 \\ 6 & -15.461539000 & 1.221198000 & 0.001852000 \\ 1 & -16.021925000 & 2.150023000 & 0.001968000 \\ 6 & -11.861885000 & -2.411929000 & 0.001196000 \\ 1 & -11.310966000 & -3.346382000 & 0.001115000 \\ 6 & 13.373676000 & 0.000016000 & -0.001048000 \\ 6 & 11.980039000 & -0.000019000 & -0.001065000 \\ 6 & 11.195532000 & 1.176055000 & -0.001022000 \\ 6 & 13.253800000 & 2.441592000 & -0.000991000 \\ 1 & 13.761296000 & 3.400542000 & -0.000965000 \\ 6 & 14.050050000 & 1.252916000 & -0.001016000 \\ 6 & 14.050096000 & -1.252856000 & -0.001028000 \\ 6 & 15.461776000 & -1.221106000 & -0.000999000 \\ 1 & 16.022184000 & -2.149927000 & -0.000984000 \\ 6 & 11.861826000 & 2.411830000 & -0.000986000 \\ 1 & 11.311159000 & 3.346420000 & -0.000953000 \\ 6 & 11.195600000 & -1.176112000 & -0.001032000 \\ 6 & 13.253904000 & -2.441560000 & -0.001015000 \\ 1 & 13.761432000 & -3.400493000 & -0.000998000 \\ 6 & 16.138577000 & 0.000072000 & -0.000983000 \\ 1 & 17.223052000 & 0.000101000 & -0.000961000 \\ 6 & 15.461718000 & 1.221233000 & -0.000987000 \\ 1 & 16.022093000 & 2.150073000 & -0.000963000 \\ 6 & 11.861919000 & -2.411858000 & -0.001009000 \\ 1 & 11.311287000 & -3.346468000 & -0.000984000\end{array}$

$\begin{array}{lccc}\mathrm{Q} 2 & (n=5) & & \\ 6 & -11.036196000 & -0.733346000 & 0.000370000 \\ 6 & 6.156363000 & -0.723725000 & -0.000445000 \\ 6 & 6.156359000 & 0.724315000 & -0.000581000 \\ 6 & -11.035977000 & 0.732613000 & -0.000150000 \\ 6 & 7.387377000 & -1.401219000 & -0.000439000 \\ 1 & 7.387310000 & -2.487120000 & -0.000331000 \\ 6 & 4.918376000 & -1.404612000 & -0.000309000 \\ 6 & 4.918481000 & 1.405201000 & -0.000673000 \\ 6 & 11.035989000 & -0.732471000 & -0.000234000 \\ 6 & 8.608024000 & 0.725435000 & -0.000635000 \\ 6 & 8.608029000 & -0.724913000 & -0.000441000 \\ 6 & 11.036194000 & 0.733495000 & -0.000735000 \\ 6 & 7.387477000 & 1.401772000 & -0.000611000 \\ 1 & 7.387377000 & 2.487678000 & -0.000740000 \\ 6 & 9.856479000 & 1.425169000 & -0.000931000\end{array}$




\begin{tabular}{|c|c|c|c|}
\hline & 9.856422000 & -1.424427000 & \\
\hline & 9.842582000 & 2.510429000 & -0.000548000 \\
\hline & 4.919625000 & -2.490353000 & -0.000164000 \\
\hline & 4.919680000 & 2.490942000 & -0.000824000 \\
\hline & 9.843525000 & -2.509671000 & 4000 \\
\hline & -6.156360000 & 0.723733000 & -0.000354000 \\
\hline U & -6.156361000 & -0.724307000 & 0.000116000 \\
\hline U & -7.387366000 & 1.401237000 & 544000 \\
\hline 1 & -7.387287000 & 2.487138000 & -0.000 \\
\hline & -4.9 & 0000 & -0.00 \\
\hline 0 & -4.91 & 3000 & 6000 \\
\hline 6 & -8.6080 & 6000 & 0.00 \\
\hline U & -8.608 & 1000 & -0.00 \\
\hline v & -7.3 & 1000 & 2000 \\
\hline 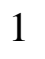 & -7.3 & 000 & 000 \\
\hline & -9.856 & -1.425 & 0.00 \\
\hline & -9.856 & 1.424 & -0.000 \\
\hline & -9.8 & -2.51 & 9000 \\
\hline & -4.9 & 000 & -0.00 \\
\hline & -4.9 & -2.4 & 0.00 \\
\hline 1 & -9.8 & 3000 & -0.00 \\
\hline 0 & 1.23 & 0.73 & -0.00 \\
\hline 0 & 1.23 & -0.7 & -0.0 \\
\hline 6 & 3.70 & -0.7 & -0.0 \\
\hline 6 & & & \\
\hline 6 & 2.45 & -1.4 & -0.0 \\
\hline 6 & 2.45 & 1.4 & -0.0 \\
\hline 1 & 2.45 & -2.4 & 0.0 \\
\hline 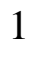 & & & \\
\hline & -0.00 & -1.4 & 0000 \\
\hline & 0.00 & 1.40 & -0.0 \\
\hline 1 & 0.00 & $-2.4 C$ & 0.00 \\
\hline . & -0.000 & & \\
\hline & -3.70 & 0.7281 & -0.000429000 \\
\hline 6 & -3.70 & -0.7286 & -0.00 \\
\hline 6 & -1.236 & -0.73 & -0.0001 \\
\hline 6 & -1.236 & 0.7328 & -0.000491000 \\
\hline 6 & -2.455 & -1.407436000 & 0.000119000 \\
\hline 6 & -2.455537000 & 1.407036000 & -0.000648000 \\
\hline 1 & -2.456844000 & -2.493137000 & 0.000419000 \\
\hline 1 & -2.457002000 & 2.492737000 & -0.000953000 \\
\hline 6 & 14.607766000 & -0.000190000 & 0.00026200 \\
\hline 6 & 13.213593000 & 0.000074000 & -0.000305000 \\
\hline 6 & 12.428880000 & -1.175724000 & -0.00104400 \\
\hline 6 & 14.487726000 & -2.441128000 & -0.00153700 \\
\hline & 14.995066000 & -3.400182000 & $-0.0018650 \mathrm{C}$ \\
\hline
\end{tabular}




\begin{tabular}{|c|c|c|c|}
\hline 6 & 15.284119000 & -1.253116000 & -0.000451000 \\
\hline 6 & 15.284722000 & 1.252454000 & 0.001758000 \\
\hline 6 & 16.696888000 & 1.220602000 & 0.002369000 \\
\hline 1 & 17.257604000 & 2.149228000 & 0.003455000 \\
\hline 6 & 13.094900000 & -2.410519000 & -0.001779000 \\
\hline 1 & 12.543619000 & -3.344823000 & -0.001911000 \\
\hline 6 & 12.429249000 & 1.176193000 & 0.000476000 \\
\hline 6 & 14.488622000 & 2.440813000 & 0.002674000 \\
\hline 1 & 14.996354000 & 3.399660000 & 0.003607000 \\
\hline 6 & 17.373433000 & -0.000817000 & 0.001618000 \\
\hline 1 & 18.457950000 & -0.000965000 & 0.002107000 \\
\hline 6 & 16.696471000 & -1.221778000 & 0.000270000 \\
\hline 1 & 17.256667000 & -2.150727000 & -0.000194000 \\
\hline 6 & 13.095861000 & 2.410717000 & 0.002114000 \\
\hline 1 & 12.544924000 & 3.345234000 & 0.002567000 \\
\hline 6 & -14.607764000 & 0.000162000 & 0.000323000 \\
\hline 6 & -13.213586000 & -0.000040000 & 0.000228000 \\
\hline 6 & -12.429172000 & -1.176132000 & 0.000700000 \\
\hline 6 & -14.488487000 & -2.440844000 & 0.001428000 \\
\hline 1 & -14.996186000 & -3.399710000 & 0.001886000 \\
\hline 6 & -15.284640000 & -1.252521000 & 0.000939000 \\
\hline 6 & -15.284202000 & 1.253046000 & -0.000195000 \\
\hline 6 & -16.696546000 & 1.221619000 & -0.000082000 \\
\hline 1 & -17.256810000 & 2.150526000 & -0.000458000 \\
\hline 6 & -13.095727000 & -2.410691000 & 0.001313000 \\
\hline 1 & -12.544743000 & -3.345179000 & 0.001683000 \\
\hline 6 & -12.428977000 & 1.175788000 & -0.000325000 \\
\hline 6 & -14.487866000 & 2.441099000 & -0.000775000 \\
\hline 1 & -14.995247000 & 3.400130000 & -0.001158000 \\
\hline 6 & -17.373436000 & 0.000606000 & 0.000517000 \\
\hline 1 & -18.457953000 & 0.000691000 & 0.000592000 \\
\hline 6 & -16.696814000 & -1.220760000 & 0.001024000 \\
\hline 1 & -17.257461000 & -2.149429000 & 0.001483000 \\
\hline 6 & -13.095034000 & 2.410547000 & -0.000835000 \\
\hline 1 & -12.543786000 & 3.344870000 & -0.001259000 \\
\hline \multicolumn{4}{|c|}{$\mathrm{Q} 2(n=5) \beta \alpha \alpha \alpha$} \\
\hline 6 & -11.034321000 & -0.733577000 & -0.000103000 \\
\hline 6 & 6.153515000 & -0.724197000 & -0.000308000 \\
\hline 6 & 6.153519000 & 0.724426000 & -0.000223000 \\
\hline 6 & -11.034256000 & 0.733335000 & 0.000170000 \\
\hline 6 & 7.385285000 & -1.401791000 & -0.000320000 \\
\hline 1 & 7.385409000 & -2.487681000 & -0.000389000 \\
\hline 6 & 4.916661000 & -1.404945000 & -0.000367000 \\
\hline 6 & 4.916743000 & 1.405197000 & -0.000150000 \\
\hline 6 & 11.033428000 & -0.733110000 & -0.000309000 \\
\hline
\end{tabular}




\begin{tabular}{|c|c|c|c|}
\hline & 8.605220000 & 0.725607000 & \\
\hline & 8.605201000 & -0.725475000 & -0.000318000 \\
\hline & 1.033514000 & 0.733422000 & -0.000100000 \\
\hline & .385362000 & 1.401976000 & -0.000217000 \\
\hline & 7.385494000 & 2.487866000 & -0.000146000 \\
\hline & 9.853776000 & 1.424947000 & \\
\hline & 9.853749000 & -1.42 & 7000 \\
\hline & 9.840420000 & & 8000 \\
\hline & 4.918148000 & -2.490692000 & -0.000450000 \\
\hline & 4.918210000 & & \\
\hline & 9.840756000 & -2.51 & \\
\hline & -6.1 & & \\
\hline & -6.155751000 & -0.724 & -0.000209000 \\
\hline & -7.385990000 & 5000 & 175000 \\
\hline & -7.385602000 & 2000 & 363000 \\
\hline & -4.9 & 1.40 & \\
\hline & -4.9 & -1.40 & -0.00 \\
\hline & -8.607 & -0.72 & 50000 \\
\hline & -8.607005000 & 0.72 & 3000 \\
\hline & -7.3 & -1.40 & -0.0 \\
\hline & -7.38 & -2.48 & -0.0 \\
\hline & -9.853210000 & -1.42 & -0.0 \\
\hline & -9.853196000 & 1.42 & 0.00 \\
\hline & -9.83 & -2.51 & -0.0 \\
\hline & -4.92 & 2.49 & 0.0 \\
\hline & -4.92 & -2.49 & -0.0 \\
\hline & -9.83 & 2.510 & 0.00 \\
\hline & 1.23 & 0.73 & -0.0 \\
\hline & 1.23 & -0.73 & -0.0 \\
\hline & 3.69 & $-0.72 \varepsilon$ & -0.0 \\
\hline & 3.699618000 & 0.728 & -0.00 \\
\hline & 2.453605000 & $-1.40^{\circ}$ & -0.0 \\
\hline & 720000 & 1.407 & -0.0 \\
\hline & 782000 & -2.49 & -0.0 \\
\hline & 2.454749 & & 03000 \\
\hline & -0.002567000 & -1.408 & -0.00 \\
\hline & -0.002422000 & 1.408 & -0.00 \\
\hline & -0.003366000 & -2.4943 & 97000 \\
\hline & -0.003461000 & 2.494467000 & 0.000078000 \\
\hline & -3.701295000 & 0.728986000 & -0.0000 \\
\hline & -3.701253000 & -0.729069000 & -0.00024800 \\
\hline & -1.235612000 & -0.733388000 & -0.000280000 \\
\hline & -1.235717000 & 0.733375000 & -0.000099000 \\
\hline & -2.457626000 & -1.407762000 & -0.00035600 \\
\hline & -2.457494000 & 1.407698000 & 0.000020000 \\
\hline & -2.459919000 & -2.493471000 & -0.00050400 \\
\hline
\end{tabular}




\begin{tabular}{|c|c|c|c|}
\hline 1 & -2.459920000 & 2.493408000 & 0.000167000 \\
\hline & 14.604805000 & -0.000097000 & 0.000208000 \\
\hline 6 & 13.210480000 & -0.000013000 & -0.000111000 \\
\hline 6 & 12.426054000 & -1.176042000 & 0.000445000 \\
\hline 6 & 14.485089000 & -2.441249000 & 0.001680000 \\
\hline 1 & 14.992657000 & -3.400193000 & 0.002232000 \\
\hline 6 & 15.281382000 & -1.252978000 & 0.001099000 \\
\hline 6 & 15.281589000 & 1.252696000 & -0.000273000 \\
\hline 6 & 16.693740000 & 1.221003000 & 0.000134000 \\
\hline 1 & 17.254285000 & 2.149750000 & -0.000188000 \\
\hline 6 & 13.092420000 & -2.410838000 & 0.001395000 \\
\hline 1 & 12.541228000 & -3.345197000 & 0.001766000 \\
\hline 6 & 12.426165000 & 1.176129000 & -0.000734000 \\
\hline 6 & 14.485366000 & 2.441091000 & -0.001027000 \\
\hline 1 & 14.993068000 & 3.399965000 & -0.001267000 \\
\hline 6 & 17.370387000 & -0.000310000 & 0.000939000 \\
\hline 1 & 18.454926000 & -0.000333000 & 0.001210000 \\
\hline 6 & 16.693625000 & -1.221446000 & 0.001415000 \\
\hline 1 & 17.253968000 & -2.150318000 & 0.002062000 \\
\hline 6 & 13.092735000 & 2.410846000 & -0.001245000 \\
\hline 1 & 12.541644000 & 3.345267000 & -0.001568000 \\
\hline 6 & -14.602469000 & -0.000006000 & 0.000155000 \\
\hline 6 & -13.208446000 & -0.000056000 & 0.000100000 \\
\hline 6 & -12.424252000 & -1.176413000 & -0.000216000 \\
\hline 6 & -14.482646000 & -2.441912000 & -0.000478000 \\
\hline 1 & -14.990462000 & -3.400690000 & -0.000694000 \\
\hline 6 & -15.278996000 & -1.252798000 & -0.000143000 \\
\hline 6 & -15.278859000 & 1.252837000 & 0.000512000 \\
\hline 6 & -16.690423000 & 1.221273000 & 0.000561000 \\
\hline 1 & -17.250792000 & 2.150103000 & 0.000825000 \\
\hline 6 & -13.091181000 & -2.412317000 & -0.000511000 \\
\hline 1 & -12.540439000 & -3.346895000 & -0.000748000 \\
\hline 6 & -12.424202000 & 1.176248000 & 0.000368000 \\
\hline 6 & -14.482489000 & 2.441883000 & 0.000791000 \\
\hline 1 & -14.990218000 & 3.400706000 & 0.001052000 \\
\hline 6 & -17.367215000 & 0.000123000 & 0.000269000 \\
\hline 1 & -18.451705000 & 0.000124000 & 0.000313000 \\
\hline 6 & -16.690489000 & -1.221151000 & -0.000077000 \\
\hline 1 & -17.251004000 & -2.149891000 & -0.000293000 \\
\hline 6 & -13.090993000 & 2.412202000 & 0.000720000 \\
\hline 1 & -12.540202000 & 3.346748000 & 0.000925000 \\
\hline \multicolumn{4}{|c|}{$\mathrm{Q} 2(n=5) \alpha \beta \alpha \alpha$} \\
\hline 6 & -11.036841000 & -0.728693000 & -0.000090000 \\
\hline 6 & 6.155044000 & -0.749111000 & -0.000297000 \\
\hline 6 & 6.150812000 & 0.701190000 & -0.000218000 \\
\hline
\end{tabular}




\begin{tabular}{|c|c|c|c|}
\hline & -11.029509000 & 0.738638000 & \\
\hline & 7.389446000 & -1.421213000 & -0.000310000 \\
\hline & 7.394022000 & -2.507008000 & 0378000 \\
\hline & 4.920721000 & -1.432960000 & -0.000350000 \\
\hline & 4.917265000 & 1.380108000 & -0.000155000 \\
\hline & 11.036772000 & -0.728432000 & -0.000280000 \\
\hline & 8.602160000 & 0.7130 & -0.0 \\
\hline & 8.608383000 & -0.738977000 & -0.00 \\
\hline & 11.029578000 & 0.738908000 & -0.000097000 \\
\hline & 7.382367000 & 1.38426 & -0.00 \\
\hline & 7.37 & 2.4702 & -0. \\
\hline & 9.84 & & -0.0 \\
\hline & 9.8587 & -1.428816000 & -0.00 \\
\hline & 9.82 & & 97000 \\
\hline & 4.9254 & -2.5186 & -0.00 \\
\hline & 4.918 & & -0.0 \\
\hline & 9.852 & -2.514 & -0.0 \\
\hline & -6.15 & & 000 \\
\hline & -6.1550 & -0.7491 & -0.00 \\
\hline & -7.3 & & \\
\hline & $-7.3^{\prime}$ & 2.470 & 0.0 \\
\hline & -4.9 & 1.380 & \\
\hline & -4.920788000 & -1.43306 & -0.0 \\
\hline & -8.6083 & -0.7390 & -0.0 \\
\hline & -8.6021 & 0.7130 & 0.0 \\
\hline & -7.389 & -1.4212 & -0. \\
\hline & -7.394073000 & -2.50706 & -0.00 \\
\hline & -9.8587 & -1.4289 & -0.0 \\
\hline & -9.8477 & 1.4217 & 0.0 \\
\hline & -9.8517 & -2.5142 & -0.0 \\
\hline & -4.918566000 & 2.46582 & 0.00 \\
\hline & -4.9255 & -2.5187 & -0.0 \\
\hline & -9.82 & 2.506 & 0.0 \\
\hline & 1.2334 & 0.7019 & -0.0 \\
\hline & 1.2342 & -0.7623 & -0.00 \\
\hline & 3.6987 & -0.7578 & -0.0 \\
\hline & 3.696 & 0.7002 & -0.00 \\
\hline & 2.4578 & -1.43769 & -0.0 \\
\hline & 2.456817000 & & -0.000120000 \\
\hline & 2.461568000 & -2.523390000 & -0.00045000 \\
\hline & 2.459488000 & 2.464468000 & -0.000026000 \\
\hline & -0.000053000 & -1.438957000 & -0.00 \\
\hline & 0.000061000 & 1.378474000 & -0.000 \\
\hline & 0.000067000 & -2.524667000 & -0.00046700 \\
\hline & -0.000059000 & & 0.000048000 \\
\hline & -3.696477000 & 0.700168000 & -0.00005600 \\
\hline
\end{tabular}




\begin{tabular}{rrrr}
6 & -3.698743000 & -0.758007000 & -0.000241000 \\
6 & -1.234155000 & -0.762451000 & -0.000272000 \\
6 & -1.233535000 & 0.701853000 & -0.000111000 \\
6 & -2.457984000 & -1.437788000 & -0.000339000 \\
6 & -2.456709000 & 1.378575000 & -0.000003000 \\
1 & -2.461518000 & -2.523481000 & -0.000471000 \\
1 & -2.459521000 & 2.464375000 & 0.000127000 \\
6 & 14.603052000 & 0.029362000 & 0.000195000 \\
6 & 13.208798000 & 0.022426000 & -0.000125000 \\
6 & 12.427924000 & -1.159791000 & 0.000374000 \\
6 & 14.493351000 & -2.414036000 & 0.001498000 \\
1 & 15.005893000 & -3.370325000 & 0.001997000 \\
6 & 15.285027000 & -1.220479000 & 0.000996000 \\
6 & 15.274238000 & 1.285235000 & -0.000191000 \\
6 & 16.687664000 & 1.259546000 & 0.000211000 \\
1 & 17.244595000 & 2.190432000 & -0.000046000 \\
6 & 13.102058000 & -2.392158000 & 0.001228000 \\
1 & 12.556517000 & -3.329768000 & 0.001571000 \\
6 & 12.421399000 & 1.192920000 & -0.000667000 \\
6 & 14.473476000 & 2.469102000 & -0.000852000 \\
1 & 14.976391000 & 3.430462000 & -0.001034000 \\
6 & 17.368192000 & 0.042127000 & 0.000929000 \\
1 & 18.452706000 & 0.046482000 & 0.001200000 \\
6 & 16.695418000 & -1.182936000 & 0.001319000 \\
1 & 17.259393000 & -2.109610000 & 0.001904000 \\
6 & 13.080547000 & 2.430953000 & -0.001076000 \\
1 & 12.524187000 & 3.362276000 & -0.001348000 \\
6 & -14.603059000 & 0.029429000 & 0.000158000 \\
6 & -13.208805000 & 0.022373000 & 0.000101000 \\
6 & -12.428004000 & -1.159918000 & -0.000179000 \\
6 & -14.493554000 & -2.413991000 & -0.000393000 \\
1 & -15.006199000 & -3.370227000 & -0.000578000 \\
6 & -15.285196000 & -1.220348000 & -0.000097000 \\
6 & -15.274083000 & 1.285366000 & 0.000473000 \\
6 & -16.687592000 & 1.259786000 & 0.000526000 \\
1 & -17.244357000 & 2.190777000 & 0.000760000 \\
6 & -13.102292000 & -2.392224000 & -0.000431000 \\
1 & -12.556815000 & -3.329874000 & -0.000640000 \\
6 & -12.421338000 & 1.192791000 & 0.000330000 \\
6 & -14.473288000 & 2.469143000 & 0.000712000 \\
6 & -14.976101000 & 3.430556000 & 0.000943000 \\
\hline & -17.368201000 & 0.042512000 & 0.000276000 \\
6 & -18.452716000 & 0.046882000 & 0.000321000 \\
6 & -17.259647000 & -2.109266000 & -0.000215000 \\
6
\end{tabular}


Q2 $(n=5) \beta \beta \alpha \alpha$

$6-11.034378000$

$-0.738196000$

$-0.000075000$

$6 \quad 6.153487000$

$-0.708811000$

$-0.000334000$

$6 \quad 6.152488000$

0.741467000

$-0.000262000$

$6 \quad-11.032284000$

0.729129000

0.000157000

$6 \quad 7.387425000$

$-1.387571000$

$-0.000341000$

$1 \quad 7.387957000$

$-2.473546000$

$-0.000396000$

$6 \quad 4.922358000$

$-1.392166000$

$-0.000380000$

$6 \quad 4.915689000$

1.420877000

$-0.000198000$

$6 \quad 11.032286000$

$-0.729090000$

$-0.000333000$

$6 \quad 8.605878000$

0.739941000

$-0.000243000$

$6 \quad 8.604854000$

$-0.712082000$

$-0.000346000$

$6 \quad 11.034379000$

0.738238000

$-0.000151000$

$6 \quad 7.384548000$

1.417888000

$-0.000255000$

17.385265000

2.503690000

$-0.000191000$

$6 \quad 9.853782000$

1.434310000

$-0.000129000$

$6 \quad 9.853027000$

$-1.416364000$

$-0.000411000$

$1 \quad 9.842989000$

2.519592000

$-0.000328000$

14.927668000

$-2.477982000$

$-0.000450000$

$1 \quad 4.916464000$

2.506557000

$-0.000130000$

$1 \quad 9.837238000$

$-2.501705000$

$-0.000241000$

$6-6.153486000$

0.708810000

$-0.000001000$

$6 \quad-6.152488000$

$-0.741468000$

$-0.000200000$

$6-7.387423000$

1.387572000

0.000136000

$1-7.387953000$

2.473546000

0.000297000

$6-4.922357000$

1.392165000

0.000053000

$6 \quad-4.915689000$

$-1.420878000$

$-0.000314000$

$\begin{array}{ll}6 & -8.605880000\end{array}$

$-0.739940000$

$-0.000143000$

$6 \quad-8.604852000$

0.712083000

0.000073000

$\begin{array}{ll}6 & -7.384548000\end{array}$

$-1.417887000$

$-0.000274000$

\section{$-7.385268000$}

$-2.503689000$

$-0.000435000$

$\begin{array}{ll}6 & -9.853789000\end{array}$

$-1.434294000$

$-0.000218000$

$6 \quad-9.853018000$

1.416380000

0.000223000

$1-9.843018000$

$-2.519575000$

$-0.000416000$

$1-4.927668000$

2.477981000

0.000197000

$1-4.916464000$

$-2.506558000$

$-0.000458000$

$1 \quad-9.837208000$

2.501722000

0.000420000

$\begin{array}{ll}6 & 1.231887000\end{array}$

0.736682000

$-0.000184000$

$\begin{array}{ll}6 & 1.235837000\end{array}$

$-0.727603000$

$-0.000312000$

$6 \quad 3.699079000$

$-0.716710000$

$-0.000328000$

$6 \quad 3.696222000$

0.741444000

$-0.000228000$

$6 \quad 2.461961000$

$-1.399754000$

$-0.000376000$

$6 \quad 2.452758000$

1.416586000

$-0.000154000$

12.468884000

$-2.485525000$

$-0.000468000$ 


\begin{tabular}{|c|c|c|c|}
\hline 1 & 2.452171000 & 2.502296000 & -0.000066000 \\
\hline & 0.005026000 & -1.408705000 & -0.000367000 \\
\hline 6 & -0.005026000 & 1.408704000 & -0.000092000 \\
\hline 1 & 0.009285000 & -2.494449000 & -0.000476000 \\
\hline 1 & -0.009284000 & 2.494448000 & 0.000015000 \\
\hline 6 & -3.699078000 & 0.716710000 & -0.000069000 \\
\hline 6 & -3.696221000 & -0.741445000 & -0.000247000 \\
\hline 6 & -1.231886000 & -0.736683000 & -0.000285000 \\
\hline 6 & -1.235836000 & 0.727603000 & -0.000130000 \\
\hline 6 & -2.452757000 & -1.416587000 & -0.000346000 \\
\hline 6 & -2.461960000 & 1.399753000 & -0.000023000 \\
\hline 1 & -2.452170000 & -2.502297000 & -0.000472000 \\
\hline 1 & -2.468884000 & 2.485524000 & 0.000103000 \\
\hline 6 & 14.603347000 & -0.007227000 & 0.000230000 \\
\hline 6 & 13.209083000 & -0.005133000 & -0.000109000 \\
\hline 6 & 12.425762000 & -1.178330000 & 0.000370000 \\
\hline 6 & 14.482259000 & -2.447358000 & 0.001537000 \\
\hline 1 & 14.988485000 & -3.406978000 & 0.002047000 \\
\hline 6 & 15.278833000 & -1.260766000 & 0.001047000 \\
\hline 6 & 15.281039000 & 1.244965000 & -0.000145000 \\
\hline 6 & 16.691483000 & 1.212328000 & 0.000288000 \\
\hline 1 & 17.252329000 & 2.140896000 & 0.000044000 \\
\hline 6 & 13.089134000 & -2.414039000 & 0.001244000 \\
\hline 1 & 12.536015000 & -3.347288000 & 0.001578000 \\
\hline 6 & 12.424072000 & 1.174369000 & -0.000671000 \\
\hline 6 & 14.485157000 & 2.435770000 & -0.000826000 \\
\hline 1 & 14.994390000 & 3.393827000 & -0.000999000 \\
\hline 6 & 17.368539000 & -0.010484000 & 0.001018000 \\
\hline 1 & 18.453062000 & -0.010993000 & 0.001311000 \\
\hline 6 & 16.692262000 & -1.230164000 & 0.001395000 \\
\hline 1 & 17.252329000 & -2.159171000 & 0.001988000 \\
\hline 6 & 13.093958000 & 2.409047000 & -0.001073000 \\
\hline 1 & 12.545142000 & 3.344746000 & -0.001362000 \\
\hline 6 & -14.603348000 & 0.007224000 & 0.000179000 \\
\hline 6 & -13.209082000 & 0.005142000 & 0.000118000 \\
\hline 6 & -12.424049000 & -1.174356000 & -0.000159000 \\
\hline 6 & -14.485125000 & -2.435774000 & -0.000361000 \\
\hline 1 & -14.994352000 & -3.393833000 & -0.000539000 \\
\hline 6 & -15.281020000 & -1.244976000 & -0.000068000 \\
\hline 6 & -15.278854000 & 1.260754000 & 0.000490000 \\
\hline 6 & -16.692281000 & 1.230129000 & 0.000546000 \\
\hline 1 & -17.252366000 & 2.159125000 & 0.000777000 \\
\hline 6 & -13.093927000 & -2.409040000 & -0.000403000 \\
\hline 1 & -12.545103000 & -3.344734000 & -0.000609000 \\
\hline 6 & -12.425793000 & 1.178345000 & 0.000339000 \\
\hline 6 & -14.482294000 & 2.447357000 & 0.000721000 \\
\hline
\end{tabular}




$\begin{array}{lrrr}1 & -14.988529000 & 3.406972000 & 0.000948000 \\ 6 & -17.368540000 & 0.010438000 & 0.000304000 \\ 1 & -18.453062000 & 0.010935000 & 0.000352000 \\ 6 & -16.691466000 & -1.212362000 & 0.000002000 \\ 1 & -17.252292000 & -2.140942000 & -0.000176000 \\ 6 & -13.089166000 & 2.414049000 & 0.000646000 \\ 1 & -12.536052000 & 3.347300000 & 0.000816000\end{array}$

$\begin{array}{lccc}\mathrm{Q} 2 & (n=5) \alpha \beta \beta \alpha & & \\ 6 & -11.032214000 & -0.733749000 & -0.000108000 \\ 6 & 6.153402000 & -0.724515000 & -0.000315000 \\ 6 & 6.153406000 & 0.724548000 & -0.000219000 \\ 6 & -11.032143000 & 0.733544000 & 0.000177000 \\ 6 & 7.384370000 & -1.401881000 & -0.000333000 \\ 1 & 7.384285000 & -2.487809000 & -0.000412000 \\ 6 & 4.917086000 & -1.405234000 & -0.000380000 \\ 6 & 4.917193000 & 1.405304000 & -0.000141000 \\ 6 & 11.032144000 & -0.733505000 & -0.000313000 \\ 6 & 8.604654000 & 0.725701000 & -0.000192000 \\ 6 & 8.604646000 & -0.725723000 & -0.000329000 \\ 6 & 11.032212000 & 0.733795000 & -0.000100000 \\ 6 & 7.384452000 & 1.401890000 & -0.000208000 \\ 1 & 7.384367000 & 2.487820000 & -0.000128000 \\ 6 & 9.851456000 & 1.425130000 & -0.000053000 \\ 6 & 9.851448000 & -1.425008000 & -0.000414000 \\ 1 & 9.837435000 & 2.510471000 & -0.000296000 \\ 1 & 4.918856000 & -2.491012000 & -0.000471000 \\ 1 & 4.918960000 & 2.491082000 & -0.000052000 \\ 1 & 9.837831000 & -2.510349000 & -0.000223000 \\ 6 & -6.153402000 & 0.724509000 & 0.000030000 \\ 6 & -6.153408000 & -0.724553000 & -0.000215000 \\ 6 & -7.384365000 & 1.401876000 & 0.000188000 \\ 1 & -7.384275000 & 2.487805000 & 0.000386000 \\ 6 & -4.917081000 & 1.405227000 & 0.000107000 \\ 6 & -4.917188000 & -1.405310000 & -0.000346000 \\ 6 & -8.604658000 & -0.725699000 & -0.000166000 \\ 6 & -8.604645000 & 0.725720000 & 0.000099000 \\ 6 & -7.384449000 & -1.401890000 & -0.000316000 \\ 1 & -7.384366000 & -2.487820000 & -0.000514000 \\ 6 & -9.851465000 & -1.425112000 & -0.000270000 \\ 6 & -9.851439000 & 1.425022000 & 0.000272000 \\ 1 & -9.837465000 & -2.510451000 & -0.000512000 \\ 1 & -4.918850000 & 2.491005000 & 0.000285000 \\ 1 & -4.918955000 & -2.491088000 & -0.000524000 \\ 1 & -9.837798000 & 2.510364000 & 0.000510000 \\ 6 & 1.235122000 & 0.733371000 & -0.000145000\end{array}$




$\begin{array}{lrrc}6 & 1.235217000 & -0.733324000 & -0.000309000 \\ 6 & 3.699586000 & -0.728904000 & -0.000318000 \\ 6 & 3.699552000 & 0.728986000 & -0.000187000 \\ 6 & 2.455393000 & -1.407587000 & -0.000384000 \\ 6 & 2.455540000 & 1.407667000 & -0.000099000 \\ 1 & 2.457388000 & -2.493308000 & -0.000501000 \\ 1 & 2.457416000 & 2.493387000 & 0.000016000 \\ 6 & -0.000085000 & -1.408926000 & -0.000383000 \\ 6 & 0.000085000 & 1.408917000 & -0.000036000 \\ 1 & 0.000021000 & -2.494612000 & -0.000520000 \\ 1 & -0.000025000 & 2.494602000 & 0.000099000 \\ 6 & -3.699581000 & 0.728898000 & -0.000035000 \\ 6 & -3.699548000 & -0.728995000 & -0.000255000 \\ 6 & -1.235110000 & -0.733382000 & -0.000287000 \\ 6 & -1.235205000 & 0.733316000 & -0.000093000 \\ 6 & -2.455538000 & -1.407677000 & -0.000369000 \\ 6 & -2.455390000 & 1.407579000 & 0.000032000 \\ 1 & -2.457416000 & -2.493397000 & -0.000527000 \\ 1 & -2.457387000 & 2.493300000 & 0.000189000 \\ 6 & 14.600692000 & -0.000021000 & 0.000209000 \\ 6 & 13.206558000 & 0.000044000 & -0.000111000 \\ 6 & 12.422298000 & -1.176323000 & 0.000465000 \\ 6 & 14.480737000 & -2.441928000 & 0.001739000 \\ 1 & 14.988469000 & -3.400765000 & 0.002314000 \\ 6 & 15.277124000 & -1.252931000 & 0.001131000 \\ 6 & 15.277307000 & 1.252817000 & -0.000300000 \\ 6 & 16.688868000 & 1.221123000 & 0.000112000 \\ 6 & -17.249416000 & 2.149856000 & -0.000230000 \\ 6 & -15.277292000 & -1.252821000 & -0.000152000 \\ 6 & 13.089148000 & -2.412140000 & 0.001448000 \\ 1 & 12.538309000 & -3.346677000 & 0.001834000 \\ 6 & 12.422409000 & 1.176478000 & -0.000757000 \\ 6 & 14.480975000 & 2.441905000 & -0.001088000 \\ 1 & 14.988814000 & 3.400685000 & -0.001348000 \\ 6 & 17.365519000 & -0.000201000 & 0.000946000 \\ 6 & 18.450034000 & -0.000216000 & 0.001221000 \\ 6 & 16.688759000 & -1.221374000 & 0.001450000 \\ 6 & 17.249117000 & -2.150224000 & 0.002119000 \\ 6 & 13.089412000 & 2.412229000 & -0.001304000 \\ 6 & -14.538640000 & 3.346808000 & -0.001648000 \\ 6 & -14250695000 & 0.000024000 & 0.000157000 \\ 6 & -0.000030000 & 0.000101000 \\ 6 & -1.252927000 & 0.000523000\end{array}$




$\begin{array}{lrrc}6 & -16.688779000 & 1.221348000 & 0.000573000 \\ 1 & -17.249156000 & 2.150187000 & 0.000845000 \\ 6 & -13.089386000 & -2.412216000 & -0.000531000 \\ 1 & -12.538605000 & -3.346791000 & -0.000775000 \\ 6 & -12.422330000 & 1.176341000 & 0.000378000 \\ 6 & -14.480772000 & 2.441933000 & 0.000813000 \\ 1 & -14.988511000 & 3.400765000 & 0.001082000 \\ 6 & -17.365524000 & 0.000164000 & 0.000271000 \\ 1 & -18.450038000 & 0.000169000 & 0.000315000 \\ 6 & -16.688855000 & -1.221149000 & -0.000086000 \\ 1 & -17.249384000 & -2.149893000 & -0.000310000 \\ 6 & -13.089180000 & 2.412153000 & 0.000741000 \\ 1 & -12.538343000 & 3.346691000 & 0.000954000\end{array}$

